\title{
A Targeting Approach To Disturbance Rejection In Multi-Agent Systems
}

\author{
by \\ Yining Liu
}

\author{
A thesis \\ presented to the University of Waterloo \\ in fulfillment of the \\ thesis requirement for the degree of \\ Master of Appilied Science \\ in \\ Electrical and Computer Engineering
}

Waterloo, Ontario, Canada, 2012

(c) Yining Liu 2012 
I hereby declare that I am the sole author of this thesis. This is a true copy of the thesis, including any required final revisions, as accepted by my examiners.

I understand that my thesis may be made electronically available to the public. 


\begin{abstract}
This thesis focuses on deadbeat disturbance rejection for discrete-time linear multi-agent systems. The multi-agent systems, on which Spieser and Shams' decentralized deadbeat output regulation problem is based, are extended by including disturbance agents. Specifically, we assume that there are one or more disturbance agents interacting with the plant agents in some known manner. The disturbance signals are assumed to be unmeasured and, for simplicity, constant. Control agents are introduced to interact with the plant agents, and each control agent is assigned a target plant agent. The goal is to drive the outputs of all plant agents to zero in finite time, despite the presence of the disturbances. In the decentralized deadbeat output regulation problem, two analysis schemes were introduced: targeting analysis, which is used to determine whether or not control laws can be found to regulate, not all the agents, but only the target agents; and growing analysis, which is used to determine the behaviour of all the non-target agents when the control laws are applied. In this thesis these two analyses are adopted to the deadbeat disturbance rejection problem. A new necessary condition for successful disturbance rejection is derived, namely that a control agent must be connected to the same plant agent to which a disturbance agent is connected. This result puts a bound on the minimum number of control agents and constraints the locations of control agents. Then, given the premise that both targeting and growing analyses succeed in the special case where the disturbances are all ignored, a new control approach is proposed for the linear case based on the idea of integral control and the regulation methods of Spieser and Shams. Preliminary studies show that this approach is also suitable for some nonlinear systems.
\end{abstract}




\section{Acknowledgements}

I would like to express my utmost gratitude to my supervisor, Professor Daniel E. Davison, for his invaluable guidance, support, and encouragement throughout my whole graduate life at the University of Waterloo.

I would also like to thank my family and friends for their love and support. Their belief in me is the source of my strength. 


\section{Table of Contents}

List of Figures

$\begin{array}{llr}1 & \text { Introduction } & 1\end{array}$

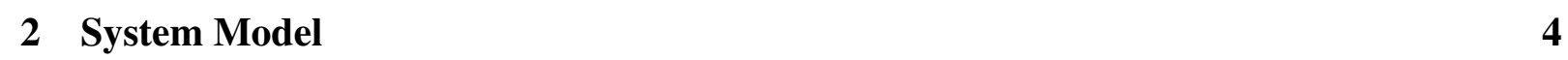

2.1 Notation and Terminology $\ldots \ldots \ldots \ldots . \ldots . \ldots . \ldots 4$

2.2 The System Model $\ldots \ldots \ldots \ldots$. . . . . . . . . . . . . . . 5

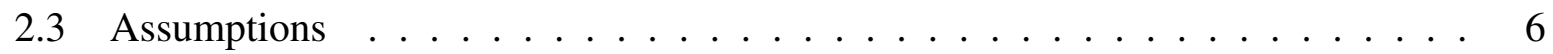

\begin{tabular}{lll}
\hline Problem Statement & 11
\end{tabular}

\begin{tabular}{|lll}
\hline & Review of Targeting And Growing Analyses & 14
\end{tabular}

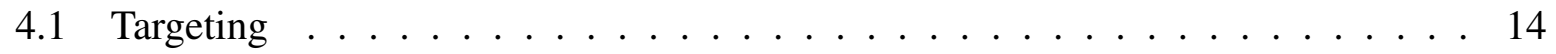

4.2 Growing . . . . . . . . . . . . . . . . . . . . . 16

4.3 Example of Targeting and Growing Analyses $\ldots \ldots \ldots$. . . . . . . . 17

5 Designing for Deadbeat Disturbance Rejection in the Linear Case 21

5.1 Necessary Conditions for Control Agent Placement . . . . . . . . . . . . . 21

5.2 Proposed Control Approach $\ldots \ldots \ldots \ldots \ldots$

5.3 Summary Of The Design Algorithm and A Detailed Example . . . . . . . . . . 36

$6 \quad$ Examples of Various Linear Multi-agent Systems 42

6.1 Ring Structure . . . . . . . . . . . . . . . . . . . . . . . . . . 42

$6.2 \quad$ Spider Structure $\ldots \ldots \ldots \ldots \ldots \ldots$ 
6.3 Grid Structure $\ldots \ldots \ldots \ldots$. . . . . . . . . . . . . . . . . . 52

6.4 Wheel Structure . . . . . . . . . . . . . . . . . . . . . 55

6.5 Complete Graph Structure . . . . . . . . . . . . . . . . . . 62

6.6 Null Graph Structure . . . . . . . . . . . . . . . . . . . . . 65

7 Deadbeat Disturbance Rejection for a Class of Nonlinear Systems 70

7.1 Three Constraints . . . . . . . . . . . . . . . . . 70

7.2 A Conjecture . . . . . . . . . . . . . . . . 75

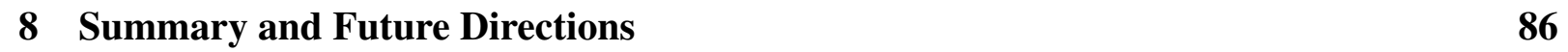

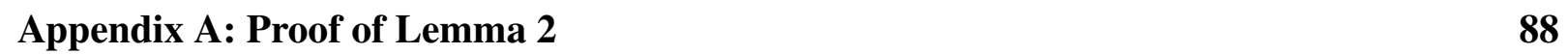

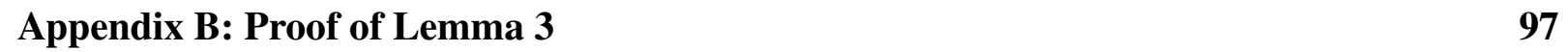

\begin{tabular}{|l|l|}
\hline Appendix C: Matlab Code for the System in Figure 5.4 & 102
\end{tabular}

\begin{tabular}{|l|l|}
\hline Appendix D: Matlab Code for the System in Figure 5.10 & 105
\end{tabular}

\begin{tabular}{lr}
\hline Bibliography & 109
\end{tabular} 


\section{List of Figures}

$2.1 \quad$ A three-agent plant with one control agent. . . . . . . . . . . . 5

2.2 A four-agent square plant with two control agents and two disturbance agents. . . 9

3.1 A three-agent queue plant with two control agents and one disturbance agent. . . 12

4.1 The dependency graph of the system in Figure 3.1$] \ldots \ldots$

4.2 The Growing Analysis Algorithm (GAA) from [17]. . . . . . . . . . . . 17

4.3 A six-agent grid plant with three control agents. . . . . . . . . . . . . 18

4.4 Simulation result for the example in Figure $4.3 \ldots \ldots \ldots$

4.5 The dependency graph of the system in Figure $4.3 \ldots \ldots \ldots$

$5.1 \quad$ Figure for the proof of Lemma $1 . \ldots \ldots \ldots \ldots \ldots$

5.2 A six-agent grid plant with two disturbance agents. . . . . . . . . . . . . 24

5.3 Control agents for a six-agent grid plant with two disturbance agents. . . . . . . . 24

5.4 A six-agent grid plant with two disturbance agents and three control agents. . . . 25

5.5 Block diagram of the proposed disturbance rejection scheme. . . . . . . . . . . 25

5.6 State-space realizations for integrators in both continuous time and discrete time. 27

5.7 Simulation result for the example in Figure 5.4$] \ldots \ldots \ldots$

5.8 Summary of the proposed disturbance rejection design algorithm. . . . . . . . 37

5.9 A six-agent queue plant with two disturbance agents. . . . . . . . . . . . . 37

5.10 Simulation results for the six-agent queue system. . . . . . . . . . . . . 41

6.1 The ring system with two disturbance agents. . . . . . . . . . . . . 43

6.2 Simulation result for the ring structure system example. . . . . . . . . . . . 45 
6.3 The spider system with six plant agents and two disturbance agents. . . . . . . . 46

6.4 Simulation result for the first spider structure system example. . . . . . . . . . . 48

6.5 The spider system with seven plant agents and two disturbance agents. . . . . . . 49

6.6 Simulation result for the second spider structure system example. . . . . . . . . . 51

6.7 The grid structure system with nine plant agents and three disturbance agents. . . 52

6.8 Simulation result for the grid structure system example. . . . . . . . . . . . 55

6.9 The first wheel structure system with ten plant agents and three disturbance agents. 56

6.10 Simulation results for the first wheel structure system example. . . . . . . . . . . 60

6.11 The second wheel structure system with five plant agents and two disturbance

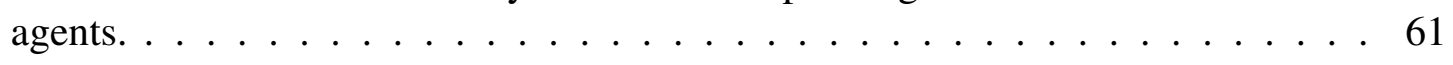

6.12 Simulation result for the second wheel structure system example. . . . . . . . . . 63

6.13 The complete graph structure system with six plant agents and three disturbance

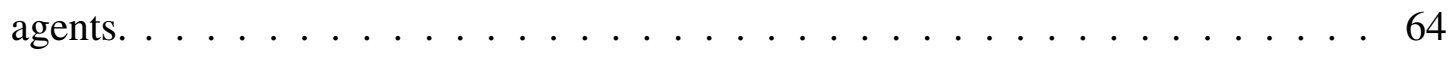

6.14 Simulation result for the complete graph structure system example. . . . . . . . . 66

6.15 The null graph structure system with six plant agents and six disturbance agents. . 67

6.16 Simulation result for the null graph structure system example. . . . . . . . . . . 69

$7.1 \quad$ A queue structure system with four plant agent and two disturbances. . . . . . . . 72

7.2 Simulation result for the queue plant with dynamics in (7.11)-(7.14). . . . . . . . 73

7.3 Simulation result for the queue plant with dynamics in (7.19)-(7.22). The simulation was aborted when $u_{6}[k]$ could not be computed. $\ldots \ldots . . . . .74$

7.4 Simulation result for the queue plant with dynamics in (7.26)-(7.29). . . . . . . . 75

7.5 Simulation result for the queue plant with dynamics in (7.34)-(7.37). . . . . . . . 78

7.6 The wheel structured nonlinear system with five plant agents and two disturbance

7.7 Simulation result for the wheel plant with dynamics in (7.46)-(7.49). . . . . . . . 81

7.8 The grid structured nonlinear system with nine plant agents and three disturbance

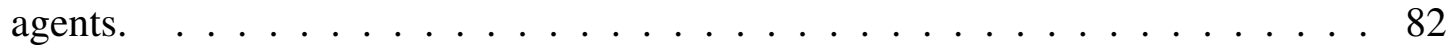

7.9 Simulation result for the wheel plant with dynamics in $(7.60)-(7.68) .2 \ldots$ 


\section{Chapter 1}

\section{Introduction}

Inspired by the psychological problem of controlling a crowd of people, earlier researchers have studied the co-operative regulation of nonlinear discrete-time multi-agent plants, where the goal is to drive the outputs of all plant agents to zero in finite time [20, 19, 16, 17]. To solve this output regulation problem, these researchers have taken the approach of placing control agents at strategic locations to interact with the plant agents. Each control agent is responsible for zeroing a single plant agent, called its target plant agent. The analysis to determine if computable control laws exist to zero all targets is called targeting analysis, which is the first stage of the solution to the regulation problem. The second stage is called growing analysis, which is used to determine whether or not the control laws zero the remaining (non-target) plant agents. The idea of targeting was first introduced to stabilize crowds modelled by suggestibility theory according to the work of Spieser and Davison (see [20], [19]). Later, Shams extended their work by allowing for arbitrary propagation time through agents and non-symmetrical influence between the agents. She also proposed several easily-interpreted necessary conditions for targeting and growing analyses to succeed [16][17]. Potential application areas of this theory include the control of unmanned autonomous vehicles, traffic control, and water management. However, one drawback of this earlier work is that disturbances are not modelled. The performance of the control schemes can be greatly degraded if disturbances are introduced.

With the above motivation, this thesis extends the regulation work by focusing on the disturbance rejection problem for discrete-time multi-agent plants. Unknown, unmeasurable, but constant disturbances are introduced to a discrete-time multi-agent plant. The new objective is deadbeat disturbance rejection, where we want all the outputs of plant agents to be zeroed in finite time despite the presence of the disturbances. Two problems are tackled. The first one deals with the question of where control agents should be placed, given knowledge of the disturbance agent locations, and the second one deals with the development of control laws (given the location of the control agents) to achieve deadbeat disturbance rejection. For the first problem, a simple but key result is that, for targeting and growing to work, a control agent must be connected 
to the same plant agent to which a disturbance agent is connected. This result implies that the number of control agents should be no less than the number of disturbance agents in the system. With the help of this result and the previous necessary conditions of Shams, it is possible to determine the minimal number of control agents needed to achieve deadbeat disturbance rejection using the targeting framework. For the second problem, a new double-loop control approach is proposed. An inner loop is based on the earlier regulation results, while an outer loop consisting of integrators and feedback are used to cancel out the disturbance effects. We show that the new disturbance rejection approach works well for linear systems, and likely for a specific class of nonlinear systems.

Although this work is theoretical, we can envision various application areas. Work on socialpsychological systems motivated this whole line of research, and provides a good illustration of how it can be used in practice. For example, consider a group of police officers trying to stabilize the psychological state of a crowd of people, while at the same time, some malicious troublemakers keep spreading rumours to influence the crowd in some other manner. To counter the malicious rumours, the police do not need to directly interact with every individual member of the crowd since people within the crowd are interacting with each other. So, instead, the police just need to interact with a few strategic people in the crowd, and focus on controlling the attitudes of a subset of the crowd; if the "right" people are chosen, then the entire crowd could conceivably be controlled. This strategy illustrates the idea of the targeting approach applied to a disturbance rejection problem. This approach may also be useful in more technical fields, such as in distributed energy generation. Consider an energy network that is composed of numerous power generators. Due to weather conditions or other random reasons, some power generators may have some problems that can be treated as disturbances. To control thousands of power generators with disturbances is a very challenging task. The new approach enables us to assign minimal control agents at the right locations, and simplify the problem. Indeed, we can envision that this work may be useful in any multi-agent problem where disturbances arise: the food distribution for chain restaurants, logistics management, mobile sensor networks, etc.

This thesis focuses on the disturbance rejection problem, but it is tightly connected to stabilization/regulation research. We introduce different control agents to interact with separate subsections of the plant network, so the results of this thesis fit into a decentralized control framework [1, 4, 18, 23]. Likewise, although not emphasized in this thesis, communication and coordination among agents relates this work to the co-operative control community [5, 6, 8, 11, 14, 13]. Other researchers have considered disturbance rejection problems arising in multi-agent systems [22, 24, 25, 9, 7], so this thesis also connects to that research field. A key strength of this work is that it provides a simple way to assign control agents in multi-agent systems with disturbances. This result is scalable to large-scale systems. Another strength, not explored in detail in this thesis, is that each control agent is not required to sense all the states of the plant agents, which helps to reduce the sensing workload per control agent when compared to centralized disturbance rejection schemes. 
A brief overview of the chapters of this thesis is given in the following. Chapter 2 first introduces notation and terminology. Then it gives the system model, extending the work of Shams [15] so that disturbance agents are incorporated. Eight assumptions are given, some identical to those in earlier work, some modified to account for disturbance rejection, and one new mild assumption introduced. This chapter provides basic knowledge for the remainder of the thesis.

Chapter 3 formalizes the concepts of deadbeat output regulation and deadbeat disturbance rejection and states the two problems being considered. As the new algorithm includes targeting and growing analyses from the regulation results, Chapter 4 reviews the analyses for multi-agent systems without disturbances. An example is given to illustrate the application of targeting and growing analyses.

The main technical contribution of this thesis is contained in Chapter 5, where a solution to the problems is provided in the case where all dynamics are linear. The results are explained with a few examples. In Chapter 6, other linear examples are given to show how this algorithm works for various network structures.

Chapter 7 attempts to extend this work to the control of nonlinear multi-agent systems. In this chapter, a class of nonlinear systems is defined by introducing three constraints. Examples are used to illustrate how these constraints were chosen. Then a conjecture is given stating that if a nonlinear multi-agent system meets the three constrains, the algorithm from Chapter 5 achieves deadbeat disturbance rejection.

Chapter 8 summarize the whole thesis with some directions for future research. Some proofs and Matlab code are given in the Appendix. 


\section{Chapter 2}

\section{System Model}

This chapter describes the model of the multi-agent plant and the associated disturbance agents. Notation and terminology are introduced, then eight assumptions are explained. Although the main results of this thesis are developed for the linear case, we provide a more general nonlinear model in this chapter.

\subsection{Notation and Terminology}

Consider a plant that is composed of $n$ agents, denoted

$$
O_{1}, \ldots, O_{n}
$$

which influence one another in a known way. In addition, $m$ control agents, denoted

$$
X_{n+1}, \ldots, X_{n+m}
$$

are introduced at specific locations with the goal of regulating the system, in the sense that the outputs of all $n$ plant agents are driven to zero in finite time. In this thesis, a plant agent whose output is driven to zero in finite time is said to be zeroed. As shown in Figure 2.1, which illustrates a three-agent plant with one control agent, directed edges are used to indicate interactions among agents. For the example in Figure 2.1, $O_{2}$ and $O_{3}$ directly influence each other, while $O_{1}$ is influenced by control agent $X_{4}$, but $X_{4}$ is not influenced by $O_{1}$. Central to our work is that each control agent $X_{i}$ is assigned a target plant agent, denoted $T_{i}$. The idea is that $X_{i}$ focuses on zeroing just its target $T_{i}$ instead of focusing simultaneously on multiple plant agents; in practical applications this targeting idea has an obvious appeal. In Figure 2.1, the target $T_{4}$ of $X_{4}$ is $O_{3}$; notice that a control agent and its target are connected by a red curved arrow. 


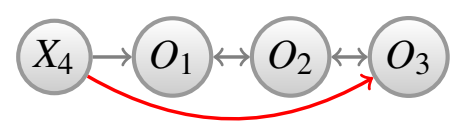

Figure 2.1: A three-agent plant with one control agent.

For the disturbance rejection problem discussed in this thesis, assume there are $p$ (with $1 \leq$ $p \leq n$ ) disturbance agents

$$
D_{1}, \ldots, D_{p}
$$

at fixed known locations in the system. For simplicity, suppose that no two disturbance agents are connected to the same plant agent, and that a control agent is not allowed to be directly connected to a disturbance agent. Hence, we can number the plant agents such that disturbance agent $D_{i}$ (for $1 \leq i \leq p$ ) is connected to plant agent $O_{i}$. Define the neighbours of $O_{i}$ (for $1 \leq i \leq n$ ), denoted $\mathcal{N}\left(O_{i}\right)$, to be the set of (plant, control, and disturbance) agents that directly influence $O_{i}$, i.e.

$$
\begin{aligned}
\mathcal{N}\left(O_{i}\right)= & \left\{O_{j}: \text { there is a directed edge from } O_{j} \text { to } O_{i}\right\} \\
\cup & \left\{X_{j}: \text { there is a directed edge from } X_{j} \text { to } O_{i}\right\} \\
\cup & \left\{D_{j}: \text { there is a directed edge from } D_{j} \text { to } O_{i}\right\} .
\end{aligned}
$$

Similarly define $\mathcal{N}\left(X_{i}\right)$ (for $\left.n+1 \leq i \leq n+m\right)$ to be the set of neighbours of $X_{i}$, i.e.

$$
\begin{aligned}
\mathcal{N}\left(X_{i}\right)= & \left\{O_{j}: \text { there is a directed edge from } O_{j} \text { to } X_{i}\right\} \\
\cup & \left\{X_{j}: \text { there is a directed edge from } X_{j} \text { to } X_{i}\right\} .
\end{aligned}
$$

In Figure 2.1, the set of neighbours of $X_{4}$ and $O_{1}$ are $\mathcal{N}\left(X_{4}\right)=\emptyset$ and $\mathcal{N}\left(O_{1}\right)=\left\{X_{4}, O_{2}\right\}$, respectively.

\subsection{The System Model}

The dynamics of the system are introduced in this section. Denote the scalar output signal of $O_{i}$ (for $1 \leq i \leq n$ ) at time $k$ by $y_{i}[k]$, and that of $D_{i}$ (for $1 \leq i \leq p$ ) by $d_{i}[k]$. Define $Y_{i}[k]$ (for $1 \leq i \leq n+m$ ) to be the set of output signals of all neighbours (except for disturbance agents) of $O_{i}$ or $X_{i}$, i.e., for plant agents

$$
Y_{i}[k]=\left\{y_{j}[k]: O_{j}[k] \in \mathcal{N}\left(O_{i}\right) \text { or } X_{j}[k] \in \mathcal{N}\left(O_{i}\right)\right\},
$$

and for control agents

$$
Y_{i}[k]=\left\{y_{j}[k]: O_{j}[k] \in \mathcal{N}\left(X_{i}\right) \text { or } X_{j}[k] \in \mathcal{N}\left(X_{i}\right)\right\}
$$


Again taking Figure 2.1 as an example, we have $Y_{2}[k]=\left\{y_{1}[k], y_{3}[k]\right\}$. Denote the state of $O_{i}$ (for $1 \leq i \leq n$ ) by $x_{i}[k]$, and the state of $D_{i}$ (for $1 \leq i \leq p$ ) by $z_{i}[k]$. Finally, denote the scalar control signal of $X_{i}$ by $u_{i}[k]$ (for $\left.n+1 \leq i \leq n+m\right)$.

The dynamics of $O_{i}$ (for $1 \leq i \leq p$ ) are taken to be

$$
\begin{aligned}
x_{i}[k+1] & =f_{i}\left(x_{i}[k], Y_{i}[k], d_{i}[k]\right) \\
y_{i}[k] & =h_{i}\left(x_{i}[k]\right),
\end{aligned}
$$

while the dynamics of $O_{i}($ for $p+1 \leq i \leq n)$ are

$$
\begin{aligned}
x_{i}[k+1] & =f_{i}\left(x_{i}[k], Y_{i}[k]\right) \\
y_{i}[k] & =h_{i}\left(x_{i}[k]\right) .
\end{aligned}
$$

Finally, the dynamics of disturbance agent $D_{i}$ (for $1 \leq i \leq p$ ) are

$$
\begin{aligned}
z_{i}[k+1] & =z_{i}[k] \\
d_{i}[k] & =z_{i}[k],
\end{aligned}
$$

that is, the disturbance $d_{i}$ is constant.

\subsection{Assumptions}

For the system with dynamics $(2.1)-(2.6)$, eight assumptions are proposed for the disturbance rejection problem. The first four assumptions are taken directly from [17]:

Assumption $A_{1}$ : There is at least one path from each control agent to its associated target.

Assumption $A_{2}$ : Control agents can communicate among themselves, with no time delays.

Assumption $A_{3}$ : Each control agent can sense the state of any plant agent or control agent.

Assumption $A_{4}$ : Each control agent targets a specific plant agent, but there are no duplicate targets. Hence, in total there are $m$ distinct targets.

These four assumptions are exploited for targeting analysis. (More details about targeting and growing analyses are reviewed in Chapter 4,) Assumption $A_{1}$ is an elementary requirement of the targeting approach, i.e., $X_{i}$ needs to be able to influence $T_{i}$ if it is to successfully zero $T_{i}$. Otherwise, targeting can never succeed, as the control agents have no way to affect the target agents. Assumption $A_{2}$ simplifies targeting analysis because an outcome of targeting analysis is a set of communication requirements among the control agents, and if we assume any communication is possible, then it is not necessary to perform additional analysis to determine, for 
example, if $X_{3}$ can communicate with $X_{8}$ via some other communication channel (say, through $X_{5}$ ). Assumption $A_{3}$ is likewise for simplification purposes. An outcome of targeting analysis is a list of states that each control agents needs to sense, and if we assume that all such combinations are possible, further analysis is not required. Finally, Assumption $A_{4}$ makes targeting analysis easier and avoids redundancy in control efforts.

The next assumption is an extension of an assumption in [17], and deals with how long it takes for signals to travel through the plant network. This mild assumption basically requires that the signal propagation times not depend on the values of the particular signals. To be more explicit, define the propagation time of $d_{i}$ through agent $O_{i}$ (for $1 \leq i \leq p$ ), denoted $\delta_{i}^{d} \geq 1$, to be the time required for a change in $d_{i}$ to propagate through the dynamics (2.1)-(2.2) to result in a change in $y_{i}$. Likewise, for any $O_{j} \in \mathcal{N}\left(O_{i}\right)$ or $X_{j} \in \mathcal{N}\left(O_{i}\right)$, define $\delta_{j i} \geq 1$ to be the time required for a change in $y_{j}$ to propagate through the dynamics $(2.1)-(2.2)$ or $(2.3)-(2.4)$ and to result in a change in $y_{i}$.

Assumption $A_{5}$ : The dynamics $\sqrt{2.1}-\sqrt{2.2}$ ) are such that the propagation time $\delta_{i}^{d}$ is independent of $d_{i}[\cdot]$. Also, the dynamics $(2.1)-(2.2)$ and $(2.3)-(2.4)$ are such that the propagation time $\delta_{j i}$ is independent of $y_{j}[\cdot]$.

Assumption $A_{5}$ implies that the propagation time along any path in the plant is constant. It is equivalent to requiring that the relative degree of the system be constant. If there is a path from $X_{i}$ to $O_{j}$ (for $n+1 \leq i \leq n+m$ and $\left.1 \leq j \leq n\right)$, say

$$
X_{i} \rightarrow O_{\alpha} \rightarrow O_{\beta} \rightarrow \cdots \rightarrow O_{\zeta} \rightarrow O_{j}
$$

denote by $\Delta\left(X_{i}, O_{j}\right)$ the propagation time required for a change in $u_{i}$ to result in a change in $y_{j}$. Evidently $\Delta\left(X_{i}, O_{j}\right)$ is the the sum of the propagation times from one agent to another along the path, i.e.,

$$
\Delta\left(X_{i}, O_{j}\right)=\Delta\left(X_{i}, O_{\alpha}\right)+\Delta\left(O_{\alpha}, O_{\beta}\right)+\Delta\left(O_{\beta}, O_{\gamma}\right)+\cdots+\Delta\left(O_{v}, O_{\zeta}\right)+\Delta\left(O_{\zeta}, O_{j}\right) .
$$

If there are multiple paths from $X_{i}$ to $O_{j}$, then $\Delta\left(X_{i}, O_{j}\right)$ is the smallest such sum. If there are no paths from $X_{i}$ to $O_{j}$, we define $\Delta\left(X_{i}, O_{j}\right)=\infty$. As an example, consider the simple queue system in Figure 2.1 again, where the control agent $X_{4}$ targets $O_{3}$. Assume the following dynamics:

$$
\begin{array}{ll}
O_{1}: & x_{1}[k+1]=x_{1}^{2}[k]+y_{2}[k]+u_{4}[k], y_{1}[k]=x_{1}[k] \\
O_{2}: & x_{2}[k+1]=y_{3}^{2}[k]+y_{1}[k], y_{2}[k]=x_{2}[k] \\
O_{3}: & x_{3}[k+1]=y_{2}[k], y_{3}[k]=x_{3}[k] .
\end{array}
$$

Assumption $A_{5}$ is satisfied with, for example, $\Delta\left(X_{4}, O_{3}\right)=\delta_{41}+\delta_{12}+\delta_{23}=1+1+1=3$.

The next assumption also appears, but in a weaker form, in [17], and is vital to the targeting approach. The assumption is related to a notion of controllability since it requires that each 
control agent, considered by itself, be able to control the output of its target. The assumption does not imply that control agents are able to simultaneously control their targets.

Assumption $A_{6}$ : For $n+1 \leq i \leq n+m$, consider control agent $X_{i}$ and its target $T_{i}=O_{j}$ (for some $1 \leq j \leq n$ ). If all control signals other than that of control agent $X_{i}$ are presumed to be known for all time, then the control signal $u_{i}[k]$ can be found (possibly dependent on the state of various plant, control, and disturbance agents at time $k$ and on the other presumed-known control signals) to force $y_{j}\left[k+\Delta\left(X_{i}, O_{j}\right)\right]=v_{i}[k]$ where $v_{i}[k] \in R$ is arbitrary but known.

As an example, consider the simple queue system in Figure 2.1 once again, with the dynamics (2.7)-(2.9). Then, Assumption $A_{6}$ is satisfied since (through iteration of the above equations)

$$
x_{3}[k+3]=x_{2}^{2}[k]+x_{1}^{2}[k]+x_{2}[k]+u_{4}[k],
$$

and therefore the control signal required for the assumption is

$$
u_{4}[k]=v_{4}[k]-x_{2}^{2}[k]-x_{1}^{2}[k]-x_{2}[k] .
$$

Note that Assumption $A_{6}$ is more restrictive than a similar assumption in [17]; the difference lies in the role of $v_{i}[k]$, which is taken to be zero in [17] but assumed to be arbitrary above. This restriction represents a cost of being able to accommodate external disturbances.

The next assumption deals with the behaviour of non-target agents. It is a slight extension of a similar assumption in [17], and it plays a central role in growing analysis. It is essentially equivalent to assuming that there are no zero dynamics in the plant.

Assumption $A_{7}$ : The dynamics of $O_{i}$ in (2.1)-(2.2) satisfy the property that, if all of the signals in $Y_{i} \cup\left\{d_{i}\right\}$ are fixed at zero, except for one (call that one $y_{j}$ ), then, for any $\bar{k} \geq 0, y_{i}[k]=0$ (for $k \geq \bar{k}$ ) implies $y_{j}[k]=0$ (for $k \geq \bar{k}$ ). Likewise, the dynamics of $O_{i}$ in $(2.3)-(2.4)$ satisfy the property that, if all of the signals in $Y_{i}$ are fixed at zero, except for one (call that one $y_{j}$ ), then, for any $\bar{k} \geq 0, y_{i}[k]=0$ (for $k \geq \bar{k}$ ) implies $y_{j}[k]=0$ (for $k \geq \bar{k}$ ).

The system in Figure 2.1 with dynamics (2.7)-(2.9) also satisfies Assumption $A_{7}$. To see this, consider, for instance, $O_{2}$ with $\mathcal{N}\left(O_{2}\right)=\left\{O_{1}, O_{3}\right\}$. If $y_{2}[k]=0$ for $k \geq \bar{k}$, (2.8) implies $y_{3}^{2}[k]+y_{1}[k]=0$ (for $k \geq \bar{k}$ ). In turn, this equation implies that if $y_{3}[\cdot]$ is zero, then necessarily $y_{1}[k]=0$ (for $k \geq \bar{k}$ ) and, conversely, if $y_{1}[\cdot]$ is zero, then necessarily $y_{3}[k]=0$ (for $k \geq \bar{k}$ ). If targeting succeeds in this example, we certainly have $y_{3}[k]=0$ (for $k \geq \bar{k}$ ). Similarly, (2.9) shows that $y_{2}[k]=0$ (for $k \geq \bar{k}$ ). So from the previous analysis, we can assure that $y_{1}[k]=0$ (for $k \geq \bar{k}$ ), which means that all the plant agents are zeroed.

Next, Assumption $A_{8}$ ensures that disturbance agents always introduce non-trivial disturbance signals. (In terms of jargon, we say that a signal $g[\cdot]$ is non-zeroed if for all $\hat{k} \geq 0$, there is a $k \geq \hat{k}$ where $g[k] \neq 0$.)

Assumption $A_{8}$ : For $1 \leq i \leq p$ and any non-zeroed $d_{i}[\cdot]$, the solution to $x_{i}[k+1]=f_{i}\left(x_{i}[k], 0, d_{i}[k]\right)$, $y_{i}[k]=h_{i}\left(x_{i}[k]\right)$ is itself non-zeroed. 


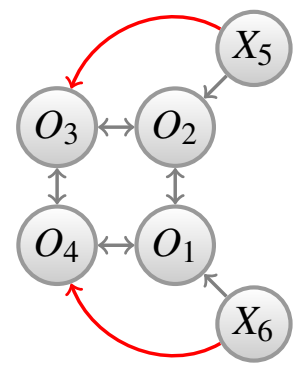

Figure 2.2: A four-agent square plant with two control agents and two disturbance agents.

Consider another example to illustrate the eight assumptions. The dynamics of the square plant in Figure 2.2 are the following:

$$
\begin{array}{lll}
O_{1} & : & x_{1}[k+1]=y_{2}[k]+2 x_{1}[k]+y_{4}[k]+u_{6}[k], y_{1}[k]=x_{1}[k] \\
O_{2} & : & x_{2}[k+1]=y_{3}[k]+3 y_{1}[k]+u_{5}[k], y_{2}[k]=x_{2}[k] \\
O_{3} & : & x_{3}[k+1]=x_{3}[k]+2 y_{2}[k]+4 y_{4}[k], y_{3}[k]=x_{3}[k] \\
O_{4} & : & x_{4}[k+1]=y_{1}[k]+y_{3}[k], y_{4}[k]=x_{4}[k] .
\end{array}
$$

First, according to the construction of the system, we notice that there is a path $X_{5} \rightarrow \mathrm{O}_{2} \rightarrow \mathrm{O}_{3}$ from $X_{5}$ to $O_{3}$ and another path $X_{6} \rightarrow O_{1} \rightarrow O_{4}$ from $X_{6}$ to $O_{4}$, which satisfies Assumption $A_{1}$. Meanwhile, there are no duplicate targets in this system, as $X_{5}$ targets $O_{3}$ and $X_{6} \operatorname{targets} O_{4}$. This confirms Assumption $A_{4}$. For Assumption $A_{5}$, the propagation times are all constants, with

$$
\begin{aligned}
& \Delta\left(X_{5}, O_{1}\right)=\delta_{52}+\delta_{21}=1+1=2 \\
& \Delta\left(X_{5}, O_{2}\right)=\delta_{52}=1 \\
& \Delta\left(X_{5}, O_{3}\right)=\delta_{52}+\delta_{23}=1+1=2 \\
& \Delta\left(X_{5}, O_{4}\right)=\delta_{52}+\delta_{23}+\delta_{34}=1+1+1=3 \\
& \Delta\left(X_{6}, O_{1}\right)=\delta_{61}=1 \\
& \Delta\left(X_{6}, O_{2}\right)=\delta_{61}+\delta_{12}=1+1=2 \\
& \Delta\left(X_{6}, O_{3}\right)=\delta_{61}+\delta_{12}+\delta_{23}=1+1+1=3 \\
& \Delta\left(X_{6}, O_{4}\right)=\delta_{61}+\delta_{14}=1+1=2 .
\end{aligned}
$$

Hence, Assumption $A_{5}$ is satisfied. Then, considering $T_{5}=O_{3}$ and the propagation time $\Delta\left(X_{5}, O_{3}\right)=$ 
2 , it is easy to determine an expression for $y_{5}[k+2]$ :

$$
\begin{aligned}
y_{3}[k+2]= & x_{3}[k+2] \\
= & x_{3}[k+1]+2 x_{2}[k+1]+4 x_{4}[k+1] \\
= & x_{3}[k]+2 x_{2}[k]+4 x_{4}[k]+2 x_{3}[k]+6 x_{1}[k]+2 u_{5}[k] \\
& +4 x_{3}[k]+4 x_{1}[k] \\
= & 10 x_{1}[k]+2 x_{2}[k]+7 x_{3}[k]+4 x_{4}[k]+2 u_{5}[k] .
\end{aligned}
$$

Setting $(2.23)$ to a given but arbitrary $v_{5}[k]$ yields

$$
u_{5}[k]=\left(v_{5}[k]-10 x_{1}[k]-2 x_{2}[k]-7 x_{3}[k]-4 x_{4}[k]\right) / 2
$$

As Assumption $A_{6}$ supposes that all the other states of various plant at time $k$ are presumed to be known for all time, it is possible to compute the control law $u_{5}[k]$. Similarly, setting the expression of $y_{4}[k+2]$ to a given but arbitrary $v_{6}[k]$ yields

$$
\begin{aligned}
y_{4}[k+2] & =x_{4}[k+2] \\
& =x_{1}[k+1]+x_{3}[k+1] \\
& =x_{3}[k]+2 x_{2}[k]+4 x_{4}[k]+x_{2}[k]+2 x_{1}[k]+x_{4}[k]+u_{6}[k] \\
& =2 x_{1}[k]+3 x_{2}[k]+x_{3}[k]+5 x_{4}[k]+u_{6}[k] \\
& =v_{6}[k]
\end{aligned}
$$

which leads to the control law of $u_{6}[k]$ :

$$
u_{6}[k]=v_{6}[k]-2 x_{1}[k]-3 x_{2}[k]-x_{3}[k]-5 x_{4}[k] .
$$

Thus, Assumption $A_{6}$ is confirmed by these expressions of the control laws.

Assumption $A_{7}$ also holds for this example. First consider $O_{1}$ with $Y_{1}=\left\{O_{2}, O_{4}\right\}$. Suppose we have $y_{4}[k]=0$ (for $k \geq \bar{k}$ ). This means that $(2.11$ becomes

$$
0=0+2 y_{2}[k]+0 .
$$

Therefore, $y_{2}[k]=0$ for any $k \geq \bar{k}$. Likewise, if $y_{2}[k]=0$ (for $k \geq \bar{k}$ ), then $y_{4}[k]=0$ (for $k \geq \bar{k}$ ). Similar arguments for $\mathrm{O}_{2}, \mathrm{O}_{3}$, and $\mathrm{O}_{4}$ verify that Assumption $\mathrm{A}_{7}$ holds.

Having stated all the assumptions for the disturbance rejection problem in multi-agent systems, we next formally pose two problems. 


\section{Chapter 3}

\section{Problem Statement}

As presented in [17], the essence of deadbeat output regulation is the following:

Deadbeat Output Regulation: A set of computable control laws $u_{n+1}, \ldots, u_{n+m}$ are said to provide deadbeat output regulation if there is a $\lambda>0$ with the property that all initial conditions on $O_{1}, \ldots, O_{n}$,

$$
y_{1}[k]=y_{2}[k]=\cdots=y_{n}[k]=0, \text { for } k \geq \lambda .
$$

In this thesis, we extend this concept to disturbance rejection:

Deadbeat Disturbance Rejection: A set of computable control laws $u_{n+1}, \ldots, u_{n+m}$ are said to provide deadbeat disturbance rejection if that there is a $\lambda>0$ with the property that for all initial conditions on $O_{1}, \ldots, O_{n}$ and for all (constant) disturbance signals $d_{1}, \ldots, d_{p}$,

$$
y_{1}[k]=y_{2}[k]=\cdots=y_{n}[k]=0, \text { for } k \geq \lambda .
$$

The definition of computable control laws is reviewed below (see Definition 1).

The two problems considered in this thesis are as follows.

Problem 1: For a given plant with known disturbance agent locations, determine how many control agents are needed, where their locations should be, and which plant agents should be chosen as their targets, in order to successfully obtain deadbeat disturbance rejection.

Problem 2: For a given plant with known disturbance agent locations and for a given set of control agent locations with known targets, find, if possible, control laws to successfully obtain deadbeat disturbance rejection.

We now review the term computable control laws, first introduced in [15] for the deadbeat output regulation problem: 


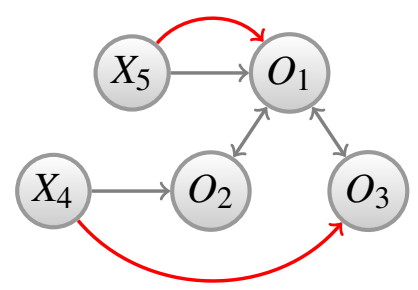

Figure 3.1: A three-agent queue plant with two control agents and one disturbance agent.

Definition 1 [15]: The control laws $u_{n+1}[\cdot], \ldots, u_{u+m}[\cdot]$ are computable if, for every time $k$, there exists a permutation of $\left(u_{n+1}[k], \ldots, u_{u+m}[k]\right)$ such that each entry (say, $\left.u_{q}[k]\right)$ in the permutation can be determined using only

(a) the values of the entries to the left of $u_{q}[k]$,

(b) control signal data at time $k-1$ or earlier, and / or

(c) state data from time $k$ or earlier.

In words, this definition requires that control laws be causal and, in cases where $m>1$, the control laws can be solved sequentially without the need for solving simultaneous (possibly nonlinear, in general) equations. Consider the example in Figure 3.1 where the dynamics of the three-agent queue system are as follows:

$$
\begin{aligned}
x_{1}[k+1] & =x_{2}[k]+x_{3}[k]+u_{5}[k] \\
y_{1}[k] & =x_{1}[k] \\
x_{2}[k+1] & =x_{1}[k]+u_{4}[k] \\
y_{2}[k] & =x_{2}[k] \\
x_{3}[k+1] & =-x_{3}[k]+2 x_{1}[k] \\
y_{3}[k] & =x_{3}[k] .
\end{aligned}
$$

Note that $\Delta\left(X_{5}, O_{1}\right)=2$ and $\Delta\left(X_{4}, O_{3}\right)=3$. We can determine control laws for $X_{4}$ and $X_{5}$ by setting $y_{3}[k+3]=0$ and $y_{1}[k+1]=0$, which results in

$$
\begin{aligned}
& u_{4}[k]=x_{2}[k]-4 x_{1}[k]+2.5 x_{3}[k]+u_{5}[k]-u_{5}[k+1] \\
& u_{5}[k]=-3 x_{1}[k-1]+x_{3}[k-1]-u_{4}[k-1] .
\end{aligned}
$$

From (3.8), we know that the control law of $X_{4}$, as written, is not computable, as the expression includes the term $u_{5}[k+1]$, which is not allowed for a computable control law. (Note that $u_{5}[k+$ 1] can be eliminated in (3.8) by substituting in for $u_{5}[k+1]$ using (3.9); however, after doing this substitution the new expression for $u_{4}[k]$ now depends on $u_{4}[k]$, which again violates the definition of computability.) 
In contrast, consider the system in Figure 2.2 with the following dynamics:

$$
\begin{aligned}
x_{1}[k+1] & =x_{2}[k]+x_{4}[k]+u_{6}[k-1] \\
y_{1}[k] & =x_{1}[k] \\
x_{2}[k+1] & =x_{1}[k]+x_{3}[k]+u_{5}[k] \\
y_{2}[k] & =x_{2}[k] \\
x_{3}[k+1] & =x_{2}[k]+x_{3}[k]+x_{4}[k] \\
y_{3}[k] & =x_{3}[k] \\
x_{4}[k+1] & =x_{1}[k]+x_{3}[k]+x_{4}[k] \\
y_{4}[k] & =x_{4}[k] .
\end{aligned}
$$

The propagation time from each agent to any of its neighbours is one, except for $\Delta\left(X_{6}, O_{1}\right)=2$, which implies that $\Delta\left(X_{5}, O_{3}\right)=2$ and $\Delta\left(X_{6}, X_{4}\right)=3$. Set $x_{3}[k+2]=0$ and $x_{4}[k+3]=0$ to yield the control laws

$$
\begin{aligned}
& u_{5}[k]=-2 x_{1}[k]-x_{2}[k]-3 x_{3}[k]-2 x_{4}[k] \\
& u_{6}[k]=-5 x_{1}[k]-3 x_{2}[k]-7 x_{3}[k]-6 x_{4}[k]-2 u_{5}[k]-u_{6}[k-1] .
\end{aligned}
$$

After substituting (3.18) into (3.19), the control laws are computable since only state information (at time $k$ and $k-1$ ) and control signal information (at time $k-1$ ) appear on the right-hand side.

Before dealing with the two problems, in Chapters 5 and 6, we first review targeting and growing analyses in the next chapter since the new algorithm for deadbeat disturbance rejection problem is based on the previous research of targeting and growing in [15]. 


\section{Chapter 4}

\section{Review of Targeting And Growing Analyses}

This chapter reviews targeting and growing analyses as presented in [17] in the context of the deadbeat regulation problem. The disturbance rejection problem is not considered here, although we heavily exploit the results of this chapter in Chapter 5. In the context of the regulation problem [17], targeting analysis is used to determine whether or not computable control laws for $X_{n+1}, \ldots, X_{n+m}$ can be found to simultaneously zero all $m$ targets 11 while growing analysis is used to determine the behaviour of all the non-target plant agents when those control laws are applied. Successful deadbeat regulation is realized if (i) targeting analysis reveals that such control laws exist, and (ii) growing analysis reveals that those control laws also zero all non-target agents. We briefly review targeting and growing analyses below.

\subsection{Targeting}

Targeting analysis proceeds, for each $n+1 \leq i \leq n+m$ and for $j$ defined by $T_{i}=O_{j}$, by iterating through the system equations to compute an expression for $y_{j}\left[k+\Delta\left(X_{i}, O_{j}\right)\right]$. Then, upon forcing $y_{j}\left[k+\Delta\left(X_{i}, O_{j}\right)\right]=0$, we can solve (by Assumption $A_{6}$ ) for $u_{i}[k]$, which will be (in general) dependent on other control signals. The goal of targeting analysis is to solve these $m$ equations for $u_{n+1}[k], \ldots, u_{n+m}[k]$; if the equations can be solved without the need to simultaneously solve (in general, nonlinear) equations, and if the control laws are causal, we say that targeting succeeds. The analysis 2.23 - 2.26) of the square system in Figure 2.2 carried out in the previous chapter

\footnotetext{
${ }^{1}$ Assumptions $A_{1}$ and $A_{6}$ guarantee that each control agent, considered by itself, is able to zero its one target. It does not follow that computable control laws can necessarily be found so that the control agents all simultaneously zero their targets.
} 
demonstrates how targeting analysis works (assuming we set all the disturbance signals to zero so that we specialize to the deadbeat regulation case).

The determination of targeting success depends on two factors: the structure of the underlying graph in the plant, and the propagation times through the plant and control agents. For a given set of control law expressions, we can decide whether the control laws are computable or not, according to Definition 1. For example, consider the three-agent queue system in Figure 3.1 described in the previous chapter; the control laws are not computable in the sense of Definition 1. As an even simpler example, consider again (2.7)-(2.9). In this example, $m=1$ so there is no need to worry about simultaneous control signals. In fact, Assumptions $A_{1}, A_{3}$ and $A_{6}$ directly imply that targeting succeeds; i.e., targeting always succeeds if $m=1$. Indeed, forcing $y_{3}[k+3]=$ 0 yields, from (2.10), the following control law:

$$
u_{4}[k]=-x_{2}^{2}[k]-x_{1}^{2}[k]-x_{2}[k]
$$

This control law zeroes the target.

In complicated large-scale systems, a more systematic approach is helpful to determine if the control laws are computable. An algorithmic approach, based on dependency graphs, is advocated in [17]. The dependency graph has nodes as the signals $u_{n+1}[\cdot], \ldots, u_{n+m}[\cdot]$ at times $\ldots, k-2, k-1, k, k+1, k+2, \ldots$, with, for each $i$ in the interval $n+1 \leq i \leq n+m$, directed edges from $u_{i}[l-1]$ to $u_{i}[l]$ (for $-\infty<l<\infty$ ) to indicate that $u_{i}[l-1]$ must be computed before $u_{i}[l]$ can be computed. In addition, directed edges are drawn, for any $j \neq i$ where $\Delta\left(X_{j}, T_{i}\right)<\infty$, to $u_{i}[l]$ from $u_{j}\left[l+\Delta\left(X_{i}, T_{i}\right)-\Delta\left(X_{j}, T_{i}\right)\right]$ (for $-\infty<l<\infty$ ) to capture the dependence of $u_{i}[\cdot]$ on other control signals [17]. For example, consider again the system in Figure 3.1. Recall the control laws derived earlier:

$$
\begin{aligned}
& u_{4}[k]=x_{2}[k]-4 x_{1}[k]+2.5 x_{3}[k]+u_{5}[k]-u_{5}[k+1] \\
& u_{5}[k]=-3 x_{1}[k-1]+x_{3}[k-1]-u_{4}[k-1] .
\end{aligned}
$$

Based on these expressions for the control laws, we can draw the dependency graph, shown in Figure 4.1. Notice that there are loops in the dependency graph, confirming the fact that the control laws in (3.8) and (3.9) are not computable. Hence, targeting does not succeed in the plant of Figure 3.1.

The following theorem, from [15], provides two necessary and sufficient tests for targeting to succeed:

Theorem 1[15]: For a given plant, given set of $m \geq 2$ control agents, and given targeting assignment, the following three conditions are equivalent:

(a) Targeting succeeds.

(b) The dependency graph has no loops.

(c) For every $p$ in the interval $2 \leq p \leq m$ and every permutation involving $p$ of the $m$ control 


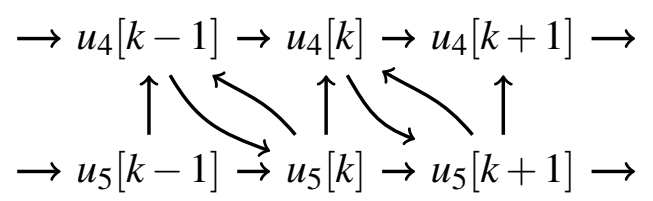

Figure 4.1: The dependency graph of the system in Figure 3.1.

agents (denoted $\left(\bar{X}_{1}, \ldots, \bar{X}_{p}\right)$ with corresponding targets $\left(\bar{T}_{1}, \ldots, \bar{T}_{p}\right)$ ),

$$
\sum_{i=1}^{p} \Delta\left(\bar{X}_{i}, \bar{T}_{i}\right)<\sum_{i=1}^{p-1} \Delta\left(\bar{X}_{i}, \bar{T}_{i+1}\right)+\Delta\left(\bar{X}_{p}, \bar{T}_{1}\right) .
$$

Condition (b) in Theorem 1 is graphical in nature, as discussed earlier. Condition (c), in contrast, is a set of algebraic constraints. The condition generates a total of $\sum_{p=2}^{m} C_{p}^{m} \cdot(p-1) !=$ $\sum_{p=2}^{m} m ! /[p(m-p) !]$ distinct inequalities. To help understand this algebraic condition better, consider the case $m=3$. In this situation, we have five inequalities in all. That is, for $p=2$, condition (c) generates three distinct inequalities, namely,

$$
\begin{aligned}
& \Delta\left(X_{1}, T_{1}\right)+\Delta\left(X_{2}, T_{2}\right)<\Delta\left(X_{1}, T_{2}\right)+\Delta\left(X_{2}, T_{1}\right) \\
& \Delta\left(X_{1}, T_{1}\right)+\Delta\left(X_{3}, T_{3}\right)<\Delta\left(X_{1}, T_{3}\right)+\Delta\left(X_{3}, T_{1}\right) \\
& \Delta\left(X_{2}, T_{2}\right)+\Delta\left(X_{3}, T_{3}\right)<\Delta\left(X_{2}, T_{3}\right)+\Delta\left(X_{3}, T_{2}\right)
\end{aligned}
$$

while for $p=3$, it generates two inequalities:

$$
\begin{aligned}
& \Delta\left(X_{1}, T_{1}\right)+\Delta\left(X_{2}, T_{2}\right)+\Delta\left(X_{3}, T_{3}\right)<\Delta\left(X_{1}, T_{2}\right)+\Delta\left(X_{2}, T_{3}\right)+\Delta\left(X_{3}, T_{1}\right) \\
& \Delta\left(X_{1}, T_{1}\right)+\Delta\left(X_{2}, T_{2}\right)+\Delta\left(X_{3}, T_{3}\right)<\Delta\left(X_{1}, T_{3}\right)+\Delta\left(X_{3}, T_{2}\right)+\Delta\left(X_{2}, T_{1}\right) .
\end{aligned}
$$

These five distinct inequalities are necessary and sufficient, according to Theorem 1 , for targeting to succeed when $m=3$.

\subsection{Growing}

If targeting succeeds, then we have (by definition) a set of computable control laws that zero all targets. We then turn to growing analysis to determine whether the control laws that resulted from targeting analysis also happen to zero non-target agents. The growing analysis algorithm (GAA) from [17], shown in Figure 4.2, determines a set of plant agents, denoted $\Omega$, that is guaranteed to be zeroed by the control laws. The set $\Omega$ "grows" as the algorithm proceeds. The GAA always terminates in a finite number of steps [17]. If $\Omega$ contains the entire set of plant agents, then we 
Step 1: Initialize $\Omega=\left\{T_{n+1}, \ldots, T_{n+m}\right\}$.

Step 2: Determine if there exists a $O_{j} \in \Omega$ such that all agents in $\mathcal{N}\left(O_{j}\right)$, except for exactly one (call it $O_{q}$ or $X_{q}$, depending on the type of agent), are elements of $\Omega$. Then necessarily $O_{q}$ (or $\left.X_{q}\right)$ is zeroed. Augment $\Omega$ with $O_{q}\left(\right.$ or $\left.X_{q}\right)$.

Step 3: Repeat Step 2 until either:

- all of $O_{1}, \ldots, O_{n}$ are in $\Omega$, in which case growing succeeds, or

- no $O_{j}$ can be found satisfying the condition of Step 2, and at least one plant agent does not appear in $\Omega$, in which case growing fails.

Figure 4.2: The Growing Analysis Algorithm (GAA) from [17].

say growing succeeds. If both targeting and growing work, then the control laws that resulted from the targeting analysis achieve plant regulation, and the settling time (i.e., the number of samples required until all plant outputs are zero) is exactly [17]

$$
\lambda=\max \left\{\Delta\left(X_{i}, T_{i}\right): n+1 \leq i \leq n+m\right\} .
$$

The following theorem summarizes these two facts about growing:

Theorem 2: [15] For a given plant, given set of $m \geq 1$ control agents, and given targeting assignment, assume targeting succeeds. Then the following hold:

(a) The GAA terminates after a finite number of iterations.

(b) If growing succeeds, then regulation of the plant is achieved with settling time $\lambda=\max \left\{\Delta\left(X_{i}, T_{i}\right)\right.$ : $n+1 \leq i \leq n+m\}$.

For the example in Figure 2.1 with dynamics (2.7)-(2.9) and control law (4.1), the GAA proceeds with $\Omega=\left\{O_{3}\right\}, \Omega=\left\{O_{3}, O_{2}\right\}$, and $\Omega=\left\{O_{3}, O_{2}, O_{1}\right\}$, and therefore growing succeeds. Thus, we conclude that the control law (4.1) achieves regulation with settling time $\lambda=3$. For the example of the square system in Figure 2.2. the GAA proceeds with $\Omega=\left\{O_{3}, O_{4}\right\}, \Omega=$ $\left\{O_{3}, O_{4}, O_{2}\right\}$, and $\Omega=\left\{O_{3}, O_{4}, O_{2}, O_{1}\right\}$, which implies that growing succeeds with settling time $\lambda=2$. This matches the analysis of the square system in Figure 2.2 in the previous chapter. Notice that, unlike targeting analysis, growing analysis depends only on the structure of the underlying plant graph [17].

\subsection{Example of Targeting and Growing Analyses}

To help further understand targeting and growing analyses, a new example in Figure 4.3 with dynamics (4.3)-(4.8) is given in this section. We go through all the steps and check out the conditions in Theorem 1 and Theorem 2. 


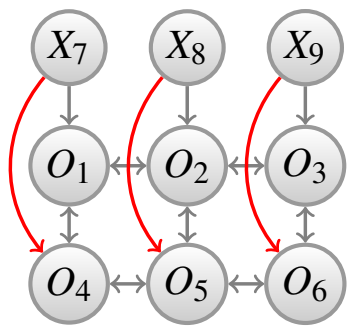

Figure 4.3: A six-agent grid plant with three control agents.

The dynamics of this example are as the following:

$$
\begin{aligned}
x_{1}[k+1] & =x_{2}[k]+x_{4}[k]+u_{7}[k-1], y_{1}[k]=x_{1}[k] \\
x_{2}[k+1] & =x_{1}[k]+x_{3}[k]+x_{5}[k]+u_{8}[k], y_{2}[k]=x_{2}[k] \\
x_{3}[k+1] & =x_{2}[k]+x_{6}[k]+u_{9}[k-1], y_{3}[k]=x_{3}[k] \\
x_{4}[k+1] & =x_{1}[k]+x_{4}[k]+x_{5}[k], y_{4}[k]=x_{4}[k] \\
x_{5}[k+1] & =x_{2}[k]+x_{4}[k]+x_{6}[k], y_{5}[k]=x_{5}[k] \\
x_{6}[k+1] & =x_{3}[k]+x_{5}[k], y_{6}[k]=x_{6}[k] .
\end{aligned}
$$

From these dynamics, we determine that the propagation time from any agent to any of its neighbours is one, except for the following cases:

$$
\begin{aligned}
& \Delta\left(X_{7}, O_{1}\right)=2 \\
& \Delta\left(X_{9}, O_{3}\right)=2 .
\end{aligned}
$$

Hence,

$$
\begin{aligned}
& \Delta\left(X_{7}, O_{4}\right)=3 \\
& \Delta\left(X_{7}, O_{5}\right)=4 \\
& \Delta\left(X_{7}, O_{6}\right)=5 \\
& \Delta\left(X_{8}, O_{4}\right)=3 \\
& \Delta\left(X_{8}, O_{5}\right)=2 \\
& \Delta\left(X_{8}, O_{6}\right)=3 \\
& \Delta\left(X_{9}, O_{4}\right)=5 \\
& \Delta\left(X_{9}, O_{5}\right)=4 \\
& \Delta\left(X_{9}, O_{6}\right)=3 .
\end{aligned}
$$

First, for targeting analysis, set the target outputs $y_{4}\left[x+\Delta\left(X_{7}, O_{4}\right)\right], y_{5}\left[x+\Delta\left(X_{8}, O_{5}\right)\right]$ and 


$$
\begin{aligned}
& y_{6}\left[x+\Delta\left(X_{9}, O_{6}\right)\right] \text { to zero as follows: } \\
& y_{4}[x+3]=x_{4}[k+3] \\
& =x_{1}[k+2]+x_{4}[k+2]+x_{5}[k+2] \\
& =x_{1}[k+1]+2 x_{2}[k+1]+3 x_{4}[k+1]+x_{5}[k+1]+x_{6}[k+1]+u_{7}[k] \\
& =5 x_{1}[k]+2 x_{2}[k]+3 x_{3}[k]+5 x_{4}[k]+6 x_{5}[k]+x_{6}[k]+u_{7}[k-1]+2 u_{8}[k]+u_{7}[k] \\
& =0 \\
& y_{5}[k+2]=x_{5}[k+2] \\
& =x_{2}[k+1]+x_{4}[k+1]+x_{6}[k+1] \\
& =2 x_{1}[k]+2 x_{3}[k]+3 x_{5}[k]+x_{4}[k]+u_{8}[k] \\
& =0 \\
& y_{6}[k+3]=x_{6}[k+3] \\
& =x_{3}[k+2]+x_{5}[k+2] \\
& =2 x_{2}[k+1]+x_{4}[k+1]+2 x_{6}[k+1]+u_{9}[k] \\
& =3 x_{1}[k]+4 x_{3}[k]+x_{4}[k]+5 x_{5}[k]+2 u_{8}[k]+u_{9}[k] \\
& =0 \text {. }
\end{aligned}
$$

From (4.9)-(4.11), we obtain the following three control laws:

$$
\begin{aligned}
& u_{7}[k]=-5 x_{1}[k]-2 x_{2}[k]-3 x_{3}[k]-5 x_{4}[k]-6 x_{5}[k]-x_{6}[k]-u_{7}[k-1]-2 u_{8}[k] \\
& u_{8}[k]=-2 x_{1}[k]-2 x_{3}[k]-x_{4}[k]-3 x_{5}[k] \\
& u_{9}[k]=-3 x_{1}[k]-4 x_{3}[k]-x_{4}[k]-5 x_{5}[k]-2 u_{8}[k] .
\end{aligned}
$$

Looking at these control laws, we see that both $u_{7}[k]$ and $u_{9}[k]$ depend on $u_{8}[k]$. The associated dependency graph, shown in Figure 4.5, has no loops. Hence, by Theorem 1, the control laws (4.12)-4.14) are computable. Alternatively, we could have used condition (c) of Theorem 1 to show that targeting succeeds. The following inequalities arise from condition (c):

$$
\begin{aligned}
\Delta\left(X_{7}, O_{4}\right)+\Delta\left(X_{8}, O_{5}\right) & <\Delta\left(X_{7}, O_{5}\right)+\Delta\left(X_{8}, O_{4}\right) \\
\Delta\left(X_{7}, O_{4}\right)+\Delta\left(X_{9}, O_{6}\right) & <\Delta\left(X_{7}, O_{6}\right)+\Delta\left(X_{9}, O_{4}\right) \\
\Delta\left(X_{8}, O_{5}\right)+\Delta\left(X_{9}, O_{6}\right) & <\Delta\left(X_{8}, O_{6}\right)+\Delta\left(X_{9}, O_{5}\right) \\
\Delta\left(X_{7}, O_{4}\right)+\Delta\left(X_{8}, O_{5}\right)+\Delta\left(X_{9}, O_{6}\right) & <\Delta\left(X_{7}, O_{5}\right)+\Delta\left(X_{8}, O_{6}\right)+\Delta\left(X_{9}, O_{4}\right) \\
\Delta\left(X_{7}, O_{4}\right)+\Delta\left(X_{8}, O_{5}\right)+\Delta\left(X_{9}, O_{6}\right) & <\Delta\left(X_{7}, O_{6}\right)+\Delta\left(X_{9}, O_{5}\right)+\Delta\left(X_{8}, O_{4}\right) .
\end{aligned}
$$

It is easy to verify that all five of these inequalities hold for this example, so we again conclude that targeting succeeds.

Next, we turn to growing analysis. It is routine to verify that Assumption $A_{7}$ holds. The GAA starts with $\Omega=\left\{\mathrm{O}_{4}, \mathrm{O}_{5}, \mathrm{O}_{6}\right\}$ and $\Omega$ grows as follows:

$$
\Omega=\left\{O_{4}, O_{5}, O_{6}, O_{1}\right\}, \Omega=\left\{O_{4}, O_{5}, O_{6}, O_{1}, O_{2}\right\}, \Omega=\left\{O_{4}, O_{5}, O_{6}, O_{1}, O_{2}, O_{3}\right\} .
$$



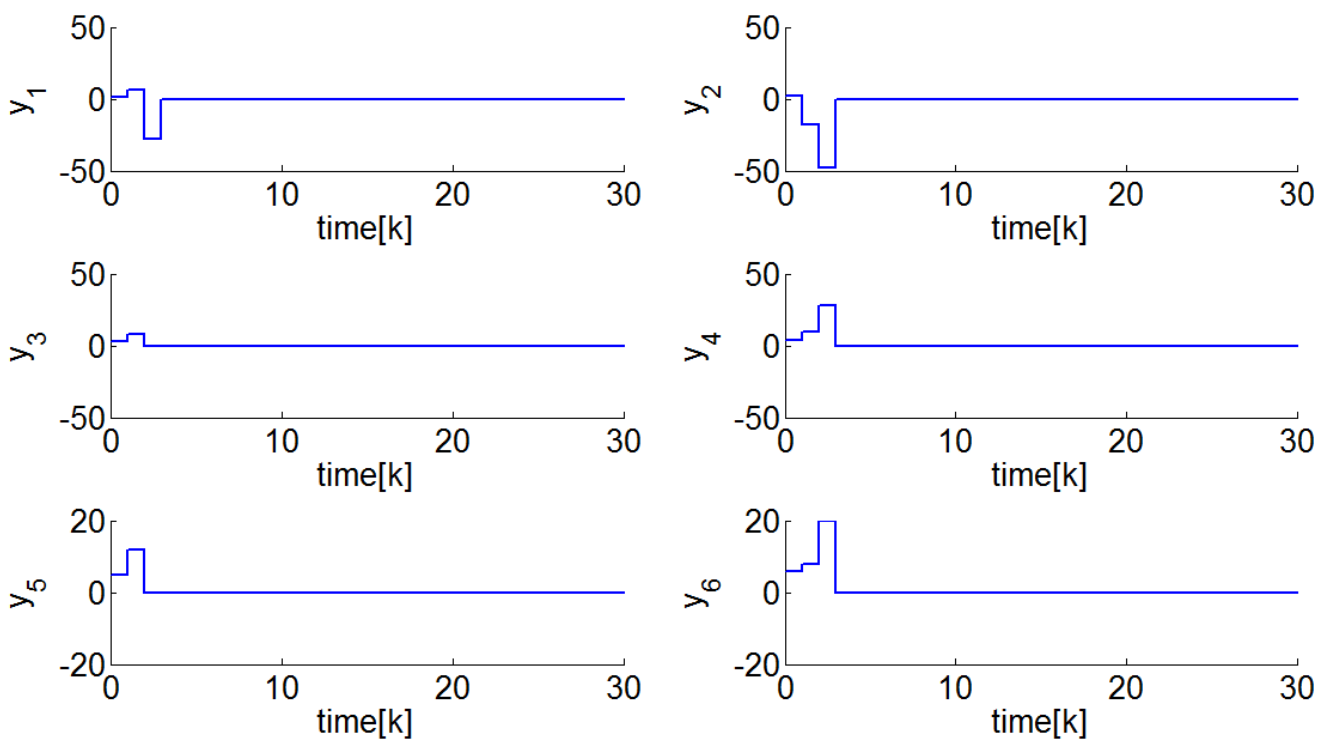

Figure 4.4: Simulation result for the example in Figure 4.3

Note that the growing process is not unique, although the final answer is unique. For this example, $\Omega$ includes all plant agents, so growing succeeds. Hence, the computable control laws (4.12)-(4.14) achieve deadbeat output regulation, as demonstrated in Figure 4.4. The settling time is $\lambda=3$.

In the next chapter, we discuss disturbance rejection for linear systems. Our approach is based on the targeting and growing analyses reviewed in this chapter, although extra complexities arise because of the presence of the disturbances.

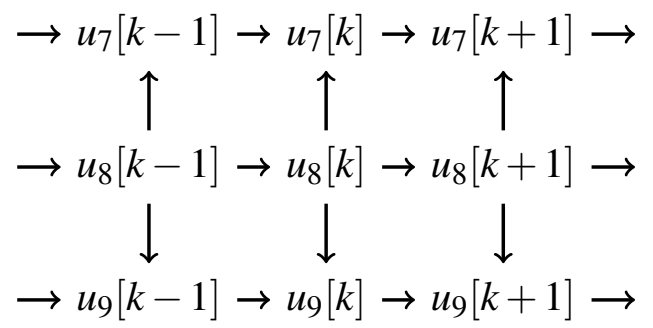

Figure 4.5: The dependency graph of the system in Figure 4.3. 


\section{Chapter 5}

\section{Designing for Deadbeat Disturbance Rejection in the Linear Case}

In this chapter, the disturbance rejection problem in the linear case is discussed. We still use the ideas of targeting and growing, but to deal with the presence of disturbances, an additional feedback loop with integral action is introduced. Figure 5.5 provides an overview of the proposed scheme, and Figure 5.8 summarizes the final design algorithm.

\subsection{Necessary Conditions for Control Agent Placement}

This section makes a contribution towards solving Problem 1, i.e., determining the number and locations of the control agents necessary to achieve deadbeat disturbance rejection. Since our approach (described below; see Figure 5.8) ultimately involves solving the regulation problem, the four necessary conditions from [17], summarized in Theorems 3 and 4 below, still apply:

Theorem 3 [17]: For a given plant, given set of $m \geq 2$ control agents, and given targeting assignment, assume that targeting succeeds. Then both the following hold:

(a) Propagation times along the paths from control agents to respective targets must be, on average, less than propagation times along the paths from control agents to all other targets.

$$
\frac{1}{m} \sum_{i=n+1}^{n+m} \Delta\left(X_{i}, T_{i}\right)<\frac{1}{m(m-1)} \sum_{i=n+1}^{n+m} \sum_{\substack{j=n+1 \\ j \neq i}}^{n+m} \Delta\left(X_{i}, T_{j}\right)
$$

(b) There are no nodes in common between a fastest path connecting $X_{i}$ to $T_{i}$ (for $n+1 \leq i \leq$ 
$n+m)$ and a fastest path connecting $X_{j}$ to $T_{j}($ for $n+1 \leq j \leq n+m, j \neq i)$.

In practice, it is probably desirable for control agents to be close to their own target agents; this condition is also desirable because it results in a small settling time. Theorem 3(a) shows that, fortuitously, this is in fact a necessary condition for targeting to succeed. We can verify that (5.1) holds for the examples given in the previous chapters. For the system in Figure 2.2, we have

$$
\begin{aligned}
& \Delta\left(X_{5}, O_{3}\right)=\delta_{52}+\delta_{23}=1+1=2 \\
& \Delta\left(X_{5}, O_{4}\right)=\delta_{52}+\delta_{23}++\delta_{34}=1+1+1=3 \\
& \Delta\left(X_{6}, O_{3}\right)=\delta_{61}+\delta_{12}+\delta_{23}=1+1+1=3 \\
& \Delta\left(X_{6}, O_{4}\right)=\delta_{61}+\delta_{14}=1+1+2
\end{aligned}
$$

and therefore

$$
\frac{1}{2}\left(\Delta\left(X_{5}, O_{3}\right)+\Delta\left(X_{6}, O_{4}\right)\right)<\frac{1}{2(2-1)}\left(\Delta\left(X_{5}, O_{4}\right)+\Delta\left(X_{6}, O_{3}\right)\right) .
$$

Thus, condition (a) of Theorem 3 is satisfied in this example. Similarly, condition (a) holds for the system in Figure 4.3

$$
\begin{array}{r}
\frac{1}{3}\left(\Delta\left(X_{7}, O_{4}\right)+\Delta\left(X_{8}, O_{5}\right)+\Delta\left(X_{9}, O_{6}\right)\right)<\frac{1}{3(3-1)}\left(\Delta\left(X_{7}, O_{5}\right)+\Delta\left(X_{7}, O_{6}\right)\right. \\
\left.+\Delta\left(X_{8}, O_{4}\right)+\Delta\left(X_{8}, O_{6}\right)+\Delta\left(X_{9}, O_{4}\right)+\Delta\left(X_{9}, O_{5}\right)\right) .
\end{array}
$$

However, condition (a) does not hold for the system in Figure 3.1 since

$$
\frac{1}{2}\left(\Delta\left(X_{5}, O_{1}\right)+\Delta\left(X_{4}, O_{3}\right)\right)=\frac{1}{2(2-1)}\left(\Delta\left(X_{5}, O_{3}\right)+\Delta\left(X_{4}, O_{1}\right)\right),
$$

which implies that targeting analysis fails. This conclusion is consistent with our earlier analysis.

Condition (b) of Theorem 3 helps to effectively reduce the number of combinations of the locations of control agents and their targets that need to be considered when solving Problem 1. It is an encouraging result because it is easy to verify from the system structure. For example, we can verify that there are no intersections among the fastest paths in both systems of Figure 2.2 and Figure 4.3. On the other hand, there is a common node $O_{1}$ between the fastest paths connecting $X_{5}$ to $O_{1}$ and $X_{4}$ to $O_{3}$ in Figure 3.1. Therefore, targeting analysis fails in this case according to condition (b). Like Theorem 3(a), this condition is also a necessary, not sufficient, condition for targeting to succeed.

Theorem 4 [17]: For a given plant, given set of $m \geq 1$ control agents, and given targeting assignment, assume that targeting succeeds. Then growing succeeds only if both the following 


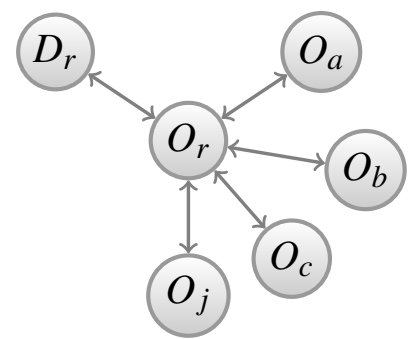

Figure 5.1: Figure for the proof of Lemma 1.

hold:

(a) Each plant agent lies on the fastest path from some $X_{i}$ to its associated $T_{i}$.

(b) For each $X_{i}$, the fastest path from $X_{i}$ to $T_{i}$ is unique.

Theorem 4 provides two necessary conditions for growing to succeed. Both of the conditions are easy to verify from the system structure, which is especially appealing when dealing with systems with large number of agents. It is easy to show that these two conditions hold in the examples in Figure 2.2, Figure 4.3, and Figure 3.1.

In addition to these four necessary conditions, we include a new necessary condition for the disturbance case:

Lemma 1: For targeting and growing to succeed, a control agent must be connected to each plant agent to which a disturbance agent is connected.

Proof: Consider a disturbance agent $D_{r}$ (for $1 \leq r \leq p$ ) connected to plant agent $O_{r}$. Suppose $O_{a}, O_{b}, O_{c}, \ldots, O_{j}$ are the plant agent neighbours of $O_{r}$, as indicated in Figure 5.1 .

We use a contradiction argument. To this end, suppose that targeting and growing succeed and that there is not a control agent connected to $O_{r}$. The dynamics of $O_{r}$ have the form (2.1)(2.2), that is,

$$
\begin{aligned}
x_{r}[k+1] & =f_{r}\left(x_{r}[k], Y_{r}[k], d_{r}[k]\right), \text { for } k \geq 0 \\
y_{r}[k] & =h_{r}\left(x_{r}[k]\right) .
\end{aligned}
$$

Since growing succeeds, all the plant agent neighbours are zeroed, i.e., $Y_{r}[k]=0$ for all $k>\bar{k}$. Consequently,

$$
\begin{aligned}
x_{r}[k+1] & =f_{r}\left(x_{r}[k], 0, d_{r}[k]\right), \text { for } k>\bar{k} \\
y_{r}[k] & =h_{r}\left(x_{r}[k]\right) .
\end{aligned}
$$

If $d_{r}[\cdot]$ is non-zeroed, then (5.4)-(5.5) imply, via Assumption $A_{7}$, that $y_{r}[\cdot]$ is non-zeroed, and therefore $O_{r}$ is not zeroed, contradicting our supposition that growing succeeds.

The necessary condition of Lemma 1, like those of Theorems 3 and 4, puts a bound on the 


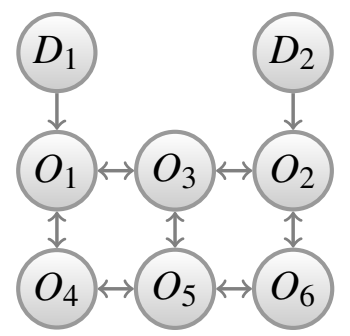

Figure 5.2: A six-agent grid plant with two disturbance agents.

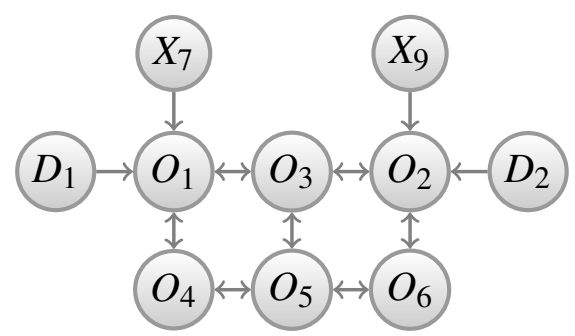

Figure 5.3: Control agents for a six-agent grid plant with two disturbance agents.

minimum number of control agents, and constrains the locations of control agents, needed to achieve deadbeat disturbance rejection. For example, Lemma 1 tells us immediately that at least as many control agents are needed as there are disturbance agents (i.e., $m \geq p$ must hold).

To illustrate the necessary conditions for Problem 1, we give an example of how to locate the control agents for the system shown in Figure 5.2. From Lemma 1, we know that control agents must be connected to plant agents to which disturbance agents are connected. This implies that at least two control agents are needed, as illustrated in Figure 5.3. We now have to choose targets for these control agents, and determine if any additional control agents are needed. For notational simplicity, assume for this example that all propagation times through individual agents are one. We quickly deduce that it is impossible to assign targets to $X_{7}$ and $X_{9}$ while satisfying condition (a) of Theorem 4 and condition (b) of Theorem 3, unless a third control agent is included. Including a control agent on $\mathrm{O}_{3}$ gives the extra flexibility to satisfy these two conditions. In fact, the conditions can be satisfied using the targeting assignment shown in Figure 5.4. Moreover, targeting and growing analyses succeed for this arrangement, so we conclude that at least three control agents are needed for this example. We will revisit this example later. 


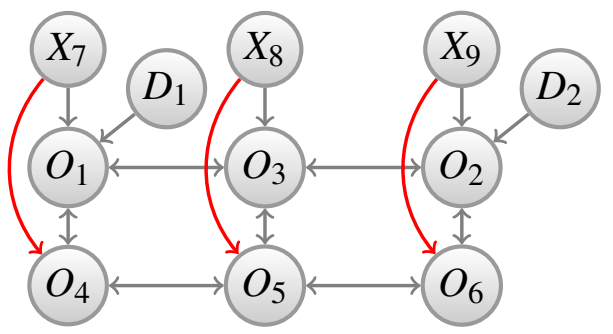

Figure 5.4: A six-agent grid plant with two disturbance agents and three control agents.

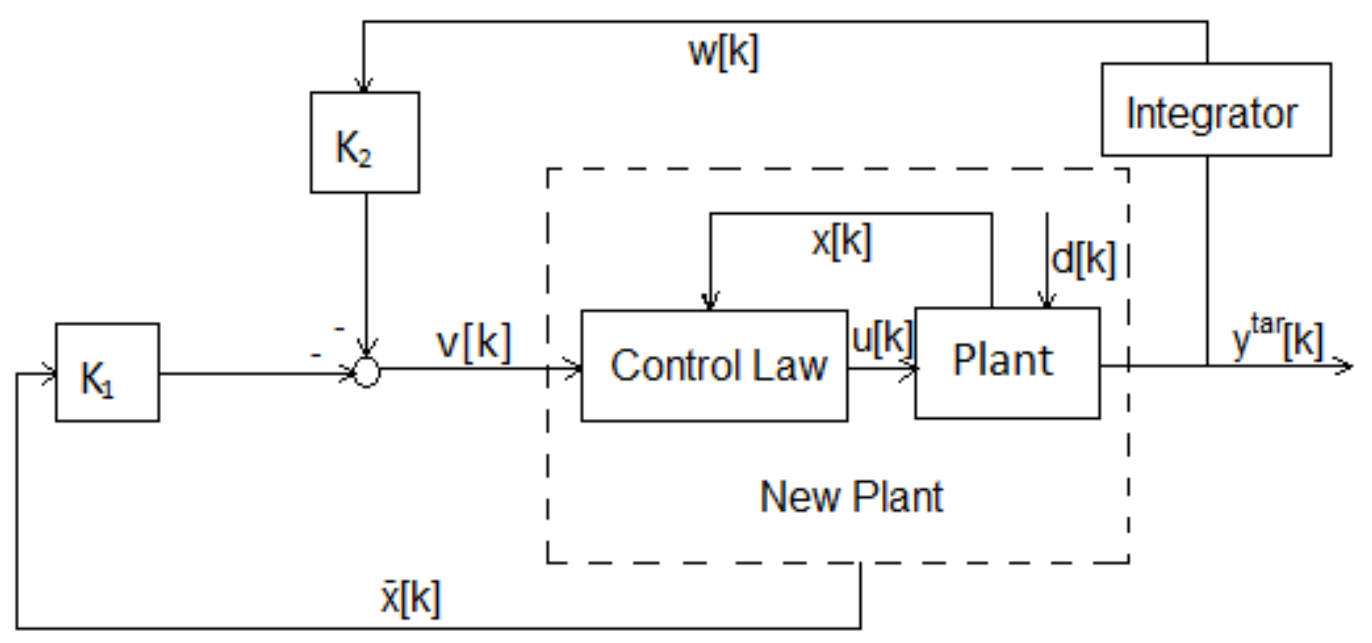

Figure 5.5: Block diagram of the proposed disturbance rejection scheme. 


\subsection{Proposed Control Approach}

Recall Problem 2 proposed in Chapter 3: For a given plant with known disturbance agent locations and for a given set of control agent locations with known targets, find, if possible, control laws to successfully obtain deadbeat disturbance rejection. This section explains a control approach to solve this problem.

A high-level block diagram of the proposed approach is presented in Figure 5.5. Focus on the dashed box. The "Plant" refers to the collection of networked plant agents, with control input

$$
u[k]=\left[u_{n+1}[k], \ldots, u_{n+m}[k]\right]^{T},
$$

disturbance input

$$
d[k]=\left[d_{1}[k], \ldots, d_{p}[k]\right]^{T},
$$

and plant state

$$
x[k]=\left[x_{1}^{T}[k], \ldots, x_{n}^{T}[k]\right]^{T} .
$$

The plant output is the set of output signals from just the target agents, namely

$$
y^{\operatorname{tar}}[k]=\left[y_{n+1}^{\operatorname{tar}}[k], \ldots, y_{n+m}^{\operatorname{tar}}[k]\right]^{T},
$$

where $y_{i}^{\text {tar }}[k]$ is the output of agent $T_{i}$ (for $n+1 \leq i \leq n+m$ ). In Figure 5.5. "Control Law" generates the control input signal $u[k]$, which is derived through targeting analysis, very much like in the regulation case, except with the introduction of signals denoted $v_{i}[k]$ to accommodate the disturbances. The dashed box can be considered as a "New Plant" which has the input

$$
v[k]=\left[v_{n+1}[k], \ldots, v_{n+m}[k]\right]^{T},
$$

the new plant states

$$
\bar{x}[k]=\left[\bar{x}_{1}^{T}[k], \ldots, \bar{x}_{n}^{T}[k]\right]^{T},
$$

and the output $y^{\operatorname{tar}}[k]$. For the integral loop in Figure 5.5, signal

$$
w[k]=\left[w_{n+1}[k], \ldots, w_{n+m}[k]\right]^{T}
$$

is the output of the "Integrator" block:

$$
w[k+1]=w[k]+y^{\operatorname{tar}}[k] .
$$

(Given the disturbance signals are constants, we use integral control to reject all disturbances in steady-state. By taking the outputs of the target agents to be the inputs of the integrators, we can expect to force the target outputs to be zero. Notice that the discrete-time integrators are different from the ones in continuous time, as shown in Figure 5.6.) 


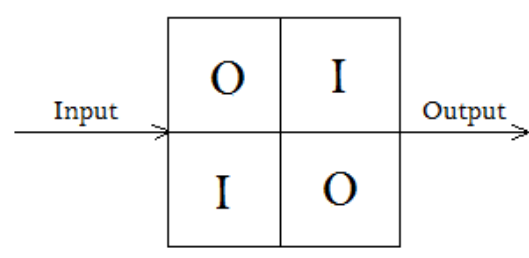

(a) Continuous Time

Integrator System

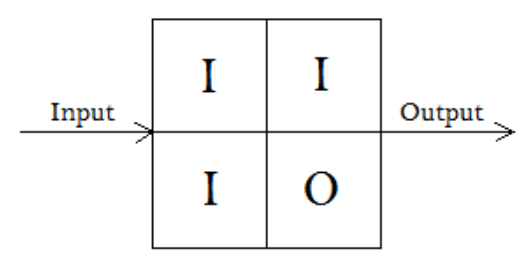

(b) Discrete Time Integrator System

Figure 5.6: State-space realizations for integrators in both continuous time and discrete time.

For simplicity, we now invoke the restriction that all the dynamics are linear. Hence, the dynamics of plant agent $O_{i}$ (for $1 \leq i \leq n$ ) can be written as

$$
\begin{aligned}
x_{i}[k+1] & =A_{i} x_{i}[k]+B_{i} y[k]+B_{i}^{u} u[k]+B_{i}^{d} d_{i}[k] \\
y_{i}[k] & =C_{i} x_{i}[k],
\end{aligned}
$$

where

$$
y[k] \triangleq\left[y_{1}[k], \ldots, y_{n}[k]\right]^{T} .
$$

If some agent does not directly influence plant agent $O_{i}$, then the associated elements of $B_{i}, B_{i}^{u}$, or $B_{i}^{d}$ are zero. For example, if there is no disturbance attached to $O_{i}$, for $p+1 \leq i \leq n$, necessarily $B_{i}^{d}=0$.

To solve Problem 2, we assume that the number and location of control agents, as well as targeting assignment, have been chosen so that

- both targeting and growing succeed in the special case where the disturbances are all zero, and

- a control agents is connected to each plant agent to which a disturbance agent is connected (as per Lemma 1).

We now use targeting analysis methods to find the control laws $u[k]$, with appropriate introduction of the signals $v[k]$ for the new plant. To this end, we note that in the special case where everything is linear, the system output $y^{t a r}[k]$ can always be expressed as (for $n+1 \leq i \leq n+m$ and where 
the various $\alpha$ coefficients are known scalars)

$$
\begin{aligned}
y_{i}^{\text {tar }}\left[k+\Delta_{i}\right] & =\sum_{n+1 \leq q \leq n+m, q \neq i}\left(\alpha_{u}^{q i} u_{q}[k]+\alpha_{d}^{q i} d_{q}[k]\right)+\alpha_{1}^{i} x_{1}[k]+\alpha_{2}^{i} x_{2}[k]+ \\
& \triangleq v_{i}[k]+\tilde{d}_{i}[k] \\
& \triangleq \tilde{v}_{i}^{i} x_{n}[k],
\end{aligned}
$$

where, for notational convenience,

$$
\Delta_{i} \triangleq \Delta\left(X_{i}, O_{i}\right), i=n+1, \ldots, n+m
$$

The sum term in 5.10$)$ includes all the control signals $u_{q}[k]$ and the disturbance signals $d_{q}[k]$, other than $u_{i}[k]$ and $d_{i}[k]$, that affect $y_{i}^{\text {tar }}\left[k+\Delta_{i}\right]$. As indicated in (5.11), we define

$$
\tilde{d}_{i}[k] \triangleq \sum_{q \neq i} \alpha_{d}^{q i} d_{q}[k]+\alpha_{d}^{i} d_{i}[k]
$$

as the net effect of disturbances on $y_{i}^{\operatorname{tar}}\left[k+\Delta_{i}\right]$, while $v_{i}[k]$ includes the effect of all the other control agents and plant agents terms. Finally, we define

$$
\tilde{v}_{i}[k] \triangleq v_{i}[k]+\tilde{d}_{i}[k]
$$

From (5.10) and (5.12), we know that

$$
\sum_{q \neq i}\left(\alpha_{u}^{q i} u_{q}[k]+\alpha_{d}^{q i} d_{q}[k]\right)+\alpha_{1}^{i} x_{1}[k]+\alpha_{2}^{i} x_{2}[k]+\cdots+\alpha_{n}^{i} x_{n}[k]+\alpha_{u}^{i} u_{i}[k]+\alpha_{d}^{i} d_{i}[k]=v_{i}[k]+\tilde{d}_{i}[k]
$$

where

$$
\tilde{d}_{i}[k] \triangleq \sum_{q \neq i} \alpha_{d}^{q i} d_{q}[k]+\alpha_{d}^{i} d_{i}[k]
$$

This implies that

$$
\sum_{q \neq i} \alpha_{u}^{q i} u_{q}[k]+\alpha_{1}^{i} x_{1}[k]+\alpha_{2}^{i} x_{2}[k]+\cdots+\alpha_{n}^{i} x_{n}[k]+\alpha_{u}^{i} u_{i}[k]=v_{i}[k]
$$

which results in the following control laws $u_{i}[k]$ (for $n+1 \leq i \leq n+m$, and assuming disturbances are zero):

$$
u_{i}[k]=\left(v_{i}[k]-\sum_{q \neq i} \alpha_{n}^{q i} u_{q}[k]-\alpha_{1}^{i} x_{1}[k]-\ldots-\alpha_{n}^{i} x_{n}[k]\right) / \alpha_{u}^{i}
$$

(The coefficient $\alpha_{u}^{i}$ is necessarily non-zero by Assumption $A_{6}$.) 
Control laws (5.14) necessarily exist because (i) we are presuming that the control agent locations and targets have been chosen to ensure that targeting analysis succeeds, and (ii) Assumption $A_{6}$ holds. Note that, if we set

$$
v_{i}[k]=0
$$

in (5.14), then the resulting control laws are exactly the same as those that would be obtained in the linear case without any disturbances.

\section{Transforming}

$$
y_{i}^{t a r}\left[k+\Delta_{i}\right]=\tilde{v}_{i}[k]
$$

into the $z$-domain results in

$$
y_{i}^{\operatorname{tar}}(z) \cdot z^{\Delta_{i}}=\tilde{v}_{i}(z) .
$$

From (5.15), we have the transfer function

$$
y_{i}^{\operatorname{tar}}(z) / \tilde{v}_{i}(z)=1 / z^{\Delta_{i}}
$$

so it follows that

$$
\left[\begin{array}{c}
y_{n+1}^{\text {tar }}(z) \\
y_{n+2}^{\text {tar }}(z) \\
\vdots \\
y_{n+m}^{\text {tar }}(z)
\end{array}\right]=\left[\begin{array}{cccc}
1 / z^{\Delta_{1}} & 0 & \cdots & 0 \\
0 & 1 / z^{\Delta_{2}} & \cdots & 0 \\
\vdots & \vdots & \ddots & \vdots \\
0 & 0 & \cdots & 1 / z^{\Delta_{m}}
\end{array}\right]\left[\begin{array}{c}
\tilde{v}_{n+1}(z) \\
\tilde{v}_{n+2}(z) \\
\vdots \\
\tilde{v}_{n+m}(z)
\end{array}\right]
$$

This system captures the dynamics of the "New Plant" in Figure 5.5. To obtain a state-space realization of this system, first recognize that, for any $r \geq 1$, a state-space realization of $1 / z^{r}$ is

$$
\begin{aligned}
A_{r}^{\prime}= & {\left[\begin{array}{ccccc}
0 & 1 & 0 & \cdots & 0 \\
0 & 0 & 1 & & 0 \\
\vdots & \vdots & \ddots & \ddots & \vdots \\
0 & 0 & \cdots & 0 & 1 \\
0 & 0 & 0 & \cdots & 0
\end{array}\right] \in R^{r \times r}, } \\
B_{r}^{\prime}= & {\left[\begin{array}{c}
0 \\
\vdots \\
0 \\
1
\end{array}\right] \in R^{r \times 1}, } \\
C_{r}^{\prime}= & {\left[\begin{array}{llll}
1 & 0 & \cdots & 0
\end{array}\right] \in R^{1 \times r}, } \\
D_{r}^{\prime}= & 0 .
\end{aligned}
$$


Thus, a state-space realization of the system in (5.17) is

$$
\begin{aligned}
\bar{x}[k+1] & =\bar{A} \bar{x}[k]+\bar{B} \tilde{v}[k] \\
& =\bar{A} \bar{x}[k]+\bar{B} v[k]+\bar{B} \tilde{d}[k] \\
y^{\operatorname{tar}}[k] & =\bar{C} \bar{x}[k],
\end{aligned}
$$

with

$$
\begin{aligned}
\bar{A} & =\operatorname{block} \operatorname{diag}\left\{A_{\Delta\left(X_{n+1}, T_{n+1}\right)}^{\prime}, \ldots, A_{\Delta\left(X_{n+m}, T_{n+m}\right)}^{\prime}\right\} \\
\bar{B} & =\operatorname{block} \operatorname{diag}\left\{B_{\Delta\left(X_{n+1}, T_{n+1}\right)}^{\prime}, \ldots, B_{\Delta\left(X_{n+m}, T_{n+m}\right)}^{\prime}\right\} \\
\bar{C} & =\operatorname{block} \operatorname{diag}\left\{C_{\Delta\left(X_{n+1}, T_{n+1}\right)}^{\prime}, \ldots, C_{\Delta\left(X_{n+m}, T_{n+m}\right)}^{\prime}\right\} .
\end{aligned}
$$

and

$$
\begin{aligned}
\bar{x}[k]= & {\left[\tilde{v}_{n+1}\left[k-\Delta_{1}\right], \tilde{v}_{n+1}\left[k-\Delta_{1}-1\right], \ldots, \tilde{v}_{n+1}[k-1], \ldots,\right.} \\
& \left.\tilde{v}_{n+m}\left[k-\Delta_{m}\right], \tilde{v}_{n+m}\left[k-\Delta_{m}-1\right], \ldots, \tilde{v}_{n+m}[k-1]\right]^{T} .
\end{aligned}
$$

A state-space realization for the augmented plant, composed of the "New Plant" (5.18)-(5.19) and the "Integrator" (5.6), is

$$
\begin{aligned}
x^{*}[k+1] & =A_{1}^{*} x^{*}[k]+B^{*}(v[k]+\tilde{d}[k]) \\
y^{\text {tar }}[k] & =C^{*} x^{*}[k],
\end{aligned}
$$

where

$$
\begin{aligned}
x^{*} & =\left[\begin{array}{c}
\bar{x}[k] \\
w[k]
\end{array}\right], \\
A_{1}^{*} & =\left[\begin{array}{cc}
\bar{A} & O \\
\bar{C} & I
\end{array}\right], \\
B^{*} & =\left[\begin{array}{c}
\bar{B} \\
0
\end{array}\right], \\
C^{*} & =\left[\begin{array}{ll}
\bar{C} & O
\end{array}\right] .
\end{aligned}
$$

We can prove that the augmented system (5.23)-(5.24) is controllable:

Lemma 2: The pair $\left(A_{1}^{*}, B^{*}\right)$ is controllable.

Proof: See Appendix A.

Lemma 2 implies that

$$
K=\left[\begin{array}{ll}
K_{1} & K_{2}
\end{array}\right]
$$


can be found so that the control law

$$
v[k]=-K_{1} \bar{x}[k]-K_{2} w[k]
$$

results in a stable deadbeat system, i.e., all closed-loop eigenvalues are at the origin. Indeed, there is a particularly simple form for the matrix $K$, dependent only on the propagation times in the plant network, that places all the eigenvalues of $A_{1}^{*}-B^{*} K$ at the origin:

Lemma 3: The following forms of matrices $K_{1}$ and $K_{2}$ place all the eigenvalues of $A_{1}^{*}-B^{*} K$ at the origin:

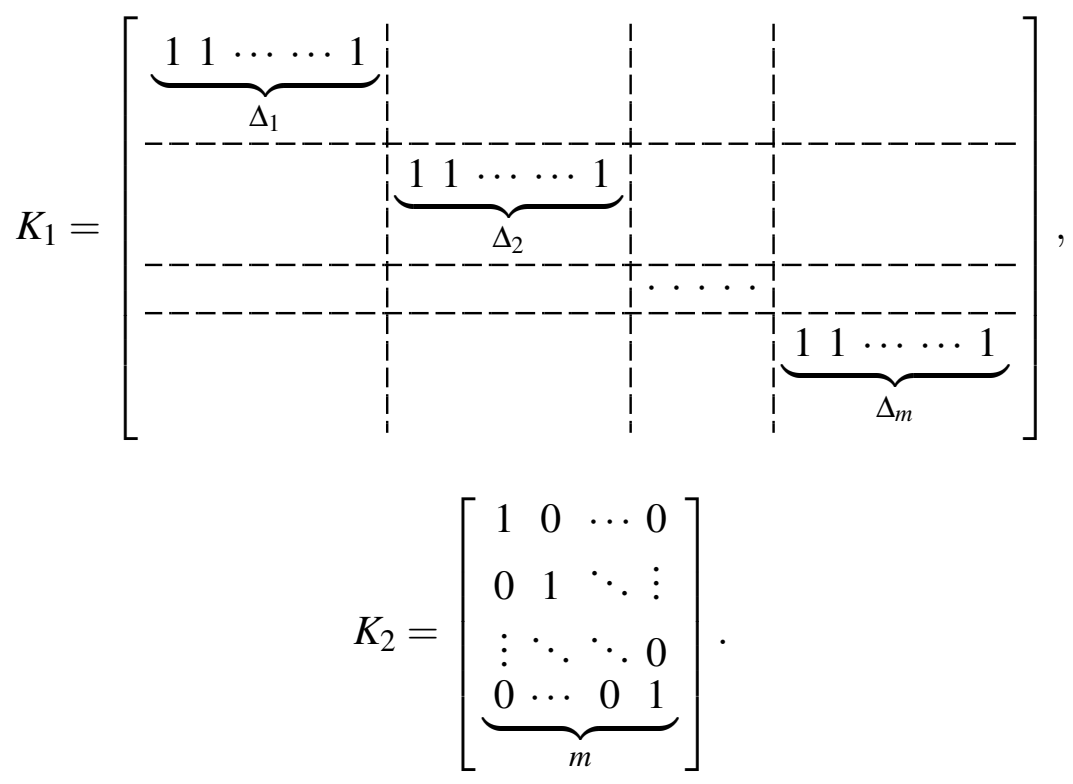

Proof: See Appendix B.

The resulting closed-loop system equations are

$$
\begin{aligned}
x^{*}[k+1] & =A^{*} x^{*}[k]+B^{*} \tilde{d}[k] ; \\
y^{\text {tar }}[k] & =C^{*} x^{*}[k],
\end{aligned}
$$

where

$$
A^{*}=\left[\begin{array}{cc}
\bar{A}-\bar{B} K_{1} & -\bar{B} K_{2} \\
\bar{C} & I
\end{array}\right] \text {. }
$$

We now obtain the main result of this thesis: 
Theorem 5: The control law obtained by substituting (5.25) into (5.14) successfully zeros, for any unknown constant disturbances, all the target agents, with the maximum settling time $\lambda=$ $m+n+\max \left\{\Delta\left(X_{i}, T_{i}\right): n+1 \leq i \leq n+m\right\}$.

Proof: By (5.28)-(5.29), we have

$$
\begin{aligned}
x^{*}(z) \cdot z & =A^{*} x^{*}(z)+B^{*} \tilde{d}(z) \\
y^{\text {tar }}(z) & =C^{*} x^{*}(z),
\end{aligned}
$$

and

$$
y^{\text {tar }}(z)=C^{*}\left(z I-A^{*}+B^{*} K\right)^{-1} B^{*} \tilde{d}(z) .
$$

As the matrix $K$ guarantees the stability of 5.30$)$, there in fact exists a steady-state value of $y^{\operatorname{tar}[\cdot], \text { namely }}$

$$
\begin{aligned}
& \lim _{k \rightarrow \infty} y^{\operatorname{tar}}[k] \\
= & \lim _{z \rightarrow 1}(z-1) y^{\operatorname{tar}}(z) \\
= & C^{*}\left(I-A^{*}+B^{*} K\right)^{-1} B^{*} \tilde{d} .
\end{aligned}
$$

Introduce matrices $S, P, T$, and $Q$ so that

$$
\begin{aligned}
& {\left[\begin{array}{ll}
S & T \\
P & Q
\end{array}\right] } \\
= & \left(I-A^{*}+B^{*} K\right)^{-1} \\
= & {\left[\begin{array}{cc}
I-\bar{A}+\bar{B} K_{1} & \bar{B} K_{2} \\
\bar{C} & 0
\end{array}\right]^{-1} . }
\end{aligned}
$$

Then,

$$
\begin{aligned}
& {\left[\begin{array}{cc}
I-\bar{A}+\bar{B} K_{1} & \bar{B} K_{2} \\
\bar{C} & 0
\end{array}\right]\left[\begin{array}{cc}
S & T \\
P & Q
\end{array}\right] } \\
= & {\left[\begin{array}{cc}
\left(I-\bar{A}+\bar{B} K_{1}\right) S+\bar{B} K_{2} P & \left(I-\bar{A}+\bar{B} K_{1}\right) T+\bar{B} K_{2} Q \\
-\bar{C} S & -\bar{C} T
\end{array}\right] } \\
= & {\left[\begin{array}{ll}
I & 0 \\
0 & I
\end{array}\right], }
\end{aligned}
$$

The $(2,1)$ entry of $(5.32)$ implies

$$
\bar{C} S=0 .
$$


Continuing from (5.31),

$$
\begin{aligned}
\lim _{k \rightarrow \infty} y^{\operatorname{tar}[k]} & =\left[\begin{array}{ll}
\bar{C} & 0
\end{array}\right]\left[\begin{array}{ll}
S & T \\
P & Q
\end{array}\right]\left[\begin{array}{l}
\bar{B} \\
0
\end{array}\right] \tilde{d} \\
& =\left[\begin{array}{ll}
\bar{C} S & \bar{C} T
\end{array}\right]\left[\begin{array}{l}
\bar{B} \\
0
\end{array}\right] \tilde{d} \\
& =\left[\begin{array}{ll}
0 & \bar{C} T
\end{array}\right]\left[\begin{array}{l}
\bar{B} \\
0
\end{array}\right] \tilde{d} \\
& =0 .
\end{aligned}
$$

Finally, the maximum settling time formula includes time steps needed to form control laws $v[k]$ in addition to the time steps for control laws $u[k]$ to zero all the targets. According to

$$
v[k]=-K_{1} \bar{x}[k]-K_{2} w[k],
$$

control laws $v[k]$ can be formed only after the $n$ entries of $\bar{x}[k]$ and the $m$ entries of $w[k]$ are known, which will take at most $n+m$ time steps. Thus considering the time which control laws $u[k]$ take to zero all the target agents, the maximum settling time is

$$
\lambda=m+n+\max \left\{\Delta\left(X_{i}, T_{i}\right): n+1 \leq i \leq n+m\right\}
$$

In summary, the control laws obtained by substituting (5.25) into $(5.14)$ zero all targets (by Lemma 1) and all non-targets (since growing succeeds), thereby achieving deadbeat disturbance rejection.

As an example, let's again consider the system in Figure 5.4. Assume the following dynamics:

$$
\begin{aligned}
x_{1}[k+1] & =x_{1}[k]+2 x_{3}[k]+x_{4}[k]+u_{7}[k]+d_{1}[k] \\
y_{1}[k] & =x_{1}[k] \\
x_{2}[k+1] & =3 x_{2}[k]+x_{3}[k]+x_{6}[k]+u_{9}[k]+d_{2}[k] \\
y_{2}[k] & =x_{2}[k] \\
x_{3}[k+1] & =x_{1}[k]+x_{2}[k]+x_{5}[k]+u_{8}[k] \\
y_{3}[k] & =x_{3}[k] \\
x_{4}[k+1] & =x_{1}[k]+x_{4}[k]+x_{5}[k] \\
y_{4}[k] & =x_{4}[k] \\
x_{5}[k+1] & =2 x_{3}[k]+x_{4}[k]+x_{6}[k]
\end{aligned}
$$




$$
\begin{aligned}
y_{5}[k] & =x_{5}[k] \\
x_{6}[k+1] & =3 x_{2}[k]+x_{5}[k]+x_{6}[k] \\
y_{6}[k] & =x_{6}[k] .
\end{aligned}
$$

Using the propagation times

$$
\begin{aligned}
& \Delta\left(X_{7}, O_{4}\right)=2 \\
& \Delta\left(X_{8}, O_{5}\right)=2 \\
& \Delta\left(X_{9}, O_{6}\right)=2
\end{aligned}
$$

we determine

$$
\begin{aligned}
x_{4}[k+2] & =x_{1}[k+1]+x_{4}[k+1]+x_{5}[k+1] \\
& =2 x_{1}[k]+4 x_{3}[k]+3 x_{4}[k]+x_{5}[k]+x_{6}[k]+u_{7}[k]+d_{1}[k] \\
& =v_{7}[k]+d_{1}[k] \\
x_{5}[k+2] & =2 x_{3}[k+1]+x_{4}[k+1]+x_{6}[k+1] \\
& =3 x_{1}[k]+5 x_{2}[k]+x_{4}[k]+4 x_{5}[k]+x_{6}[k]+2 u_{8}[k] \\
& =v_{8}[k] \\
x_{6}[k+2] & =3 x_{2}[k+1]+x_{5}[k+1]+x_{6}[k+1] \\
& =12 x_{2}[k]+5 x_{3}[k]+x_{4}[k]+5 x_{6}[k]+x_{5}[k]+3 u_{9}[k]+3 d_{2}[k] \\
& =v_{9}[k]+3 d_{2}[k],
\end{aligned}
$$

which have the form of (5.10) and (5.12). From (5.46) - (5.48), the control laws of $u_{7}, u_{8}$ and $u_{9}$ are

$$
\begin{aligned}
& u_{7}[k]=v_{7}[k]-2 x_{1}[k]-4 x_{3}[k]-3 x_{4}[k]-x_{5}[k]-x_{6}[k] \\
& u_{8}[k]=\left(v_{8}[k]-3 x_{1}[k]-5 x_{2}[k]-x_{4}[k]-4 x_{5}[k]-x_{6}[k]\right) / 2 \\
& u_{9}[k]=\left(v_{9}[k]-12 x_{2}[k]-5 x_{3}[k]-x_{4}[k]-x_{5}[k]-5 x_{6}[k]\right) / 3 .
\end{aligned}
$$

Next, construct the augmented system (5.23)-(5.24):

$$
\begin{aligned}
x^{*}[k+1] & =A_{1}^{*} x^{*}[k]+B^{*}(v[k]+\tilde{d}[k]) \\
y^{\text {tar }}[k] & =C^{*} x^{*}[k],
\end{aligned}
$$


where

$$
\begin{aligned}
x^{*} & =\left[\begin{array}{c}
\bar{x}[k] \\
w[k]
\end{array}\right], \\
A_{1}^{*} & =\left[\begin{array}{ll}
\bar{A} & O \\
\bar{C} & I
\end{array}\right], \\
B^{*} & =\left[\begin{array}{l}
\bar{B} \\
0
\end{array}\right], \\
C^{*} & =\left[\begin{array}{ll}
\bar{C} & O
\end{array}\right],
\end{aligned}
$$

with

$$
\begin{aligned}
\bar{A} & =\operatorname{block} \operatorname{diag}\left\{A_{\Delta\left(X_{7}, O_{2}\right)}^{\prime}, A_{\Delta\left(X_{8}, O_{5}\right)}^{\prime}, A_{\Delta\left(X_{9}, O_{6}\right)}^{\prime}\right\} \\
& =\text { block } \operatorname{diag}\left\{A_{2}^{\prime}, A_{2}^{\prime}, A_{2}^{\prime}\right\} \\
\bar{B} & =\operatorname{block} \operatorname{diag}\left\{B_{\Delta\left(X_{7}, O_{2}\right)}^{\prime}, B_{\Delta\left(X_{8}, O_{5}\right)}^{\prime}, B_{\Delta\left(X_{9}, O_{6}\right)}^{\prime}\right\} \\
& =\operatorname{block} \operatorname{diag}\left\{B_{2}^{\prime}, B_{2}^{\prime}, B_{2}^{\prime}\right\} \\
\bar{C} & =\operatorname{block} \operatorname{diag}\left\{C_{\Delta\left(X_{7}, O_{2}\right)}^{\prime}, C_{\Delta\left(X_{8}, O_{5}\right)}^{\prime}, C_{\Delta\left(X_{9}, O_{6}\right)}^{\prime}\right\}, \\
& =\text { block } \operatorname{diag}\left\{C_{2}^{\prime}, C_{2}^{\prime}, C_{2}^{\prime}\right\},
\end{aligned}
$$

and

$$
A_{2}^{\prime}=\left[\begin{array}{ll}
0 & 1 \\
0 & 0
\end{array}\right], B_{2}^{\prime}=\left[\begin{array}{l}
0 \\
1
\end{array}\right], C_{2}^{\prime}=\left[\begin{array}{ll}
1 & 0
\end{array}\right]
$$

Using the propagation times from the three control agents to their targets, we use Lemma 3 to find $K_{1}$ and $K_{2}$ to place all eigenvalues of $A_{1}^{*}-B^{*} K$ at the origin:

$$
K_{1}=\left[\begin{array}{llllll}
1 & 1 & 0 & 0 & 0 & 0 \\
0 & 0 & 1 & 1 & 0 & 0 \\
0 & 0 & 0 & 0 & 1 & 1
\end{array}\right], K_{2}=\left[\begin{array}{lll}
1 & 0 & 0 \\
0 & 1 & 0 \\
0 & 0 & 1
\end{array}\right] .
$$

The resulting control laws for $v[k]$ are

$$
\begin{aligned}
& v_{7}[k]=-\bar{x}_{1}[k]-\bar{x}_{2}[k]-w_{1}[k] \\
& v_{8}[k]=-\bar{x}_{3}[k]-\bar{x}_{4}[k]-w_{2}[k] \\
& v_{9}[k]=-\bar{x}_{5}[k]-\bar{x}_{6}[k]-w_{3}[k] .
\end{aligned}
$$

Finally, substitute $v[k]$ into 5.49$]-(5.51)$ to complete the controller design

$$
\begin{aligned}
u_{7}[k] & =-\bar{x}_{1}[k]-\bar{x}_{2}[k]-w_{1}[k]-2 x_{1}[k]-4 x_{3}[k]-3 x_{4}[k]-x_{5}[k]-x_{6}[k] \\
u_{8}[k] & =\left(-\bar{x}_{3}[k]-\bar{x}_{4}[k]-w_{2}[k]-3 x_{1}[k]-5 x_{2}[k]-x_{4}[k]-4 x_{5}[k]-x_{6}[k]\right) / 2 \\
u_{9}[k] & =\left(-\bar{x}_{5}[k]-\bar{x}_{6}[k]-w_{3}[k]-12 x_{2}[k]-5 x_{3}[k]-x_{4}[k]-x_{5}[k]-5 x_{6}[k]\right) / 3,
\end{aligned}
$$



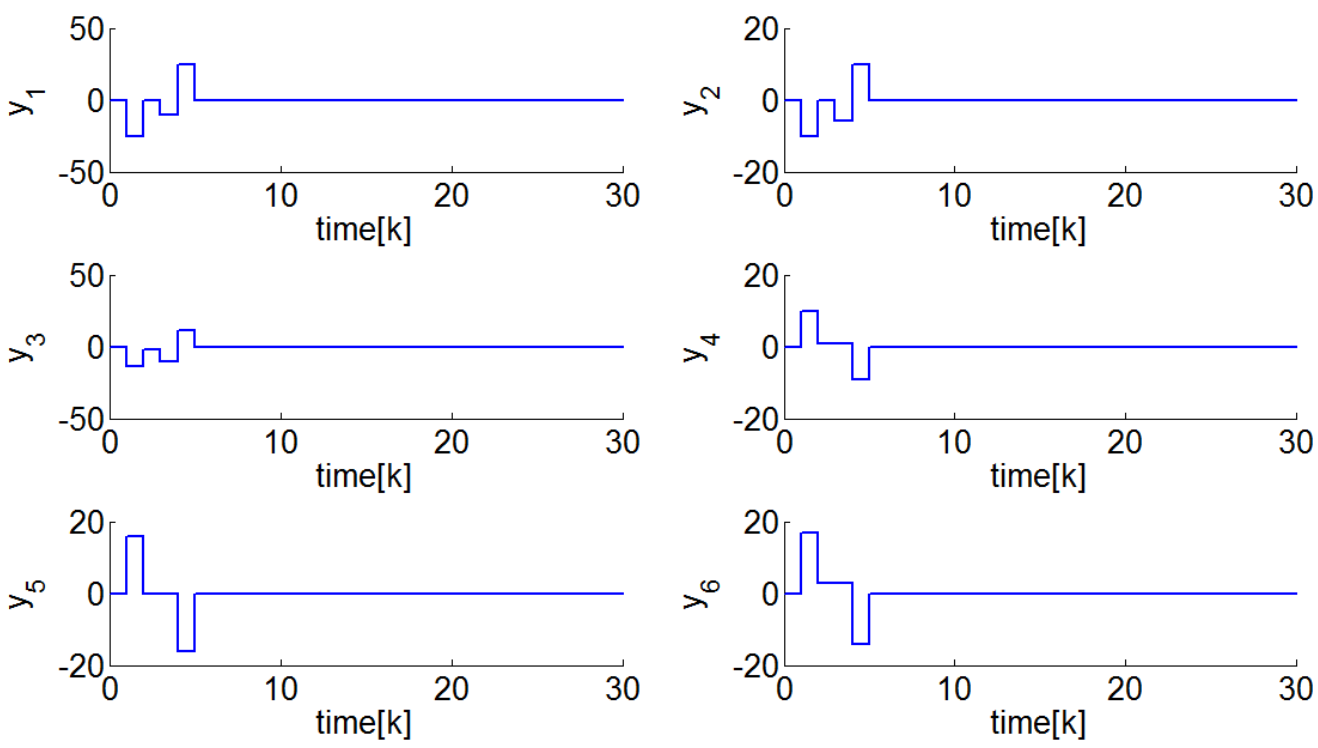

Figure 5.7: Simulation result for the example in Figure 5.4

where

$$
\bar{x}[k]=\left[v_{7}[k-2], v_{7}[k-1], v_{8}[k-2], v_{8}[k-1], v_{9}[k-2], v_{9}[k-1]\right]^{T} .
$$

Matlab code for implementation of the controller is shown in Appendix C. Using the initial conditions and disturbances

$$
\begin{aligned}
x[0] & =\left[\begin{array}{llllll}
1 & 2 & 3 & 4 & 5 & 6
\end{array}\right]^{T}, \\
\bar{x}[0] & =\left[\begin{array}{llllll}
0 & 0 & 0 & 0 & 0 & 0
\end{array}\right]^{T} \\
w[0] & =\left[\begin{array}{lll}
0 & 0 & 0
\end{array}\right]^{T} \\
d_{1}[k] & =d_{2}[k]=1(\text { for } k \geq 0),
\end{aligned}
$$

simulations (see Figure 5.7) show that the control laws successfully reject constant disturbances.

\subsection{Summary Of The Design Algorithm and A Detailed Ex- ample}

The proposed disturbance rejection design algorithm is summarized in Figure 5.8. As a detailed example, consider the queue system in Figure 5.9. The dynamic equations of this system, with 
Step 1: Choose the number of control agents, their placement, and targeting assignment to be consistent with Lemma 1 and such that both targeting and growing succeed. Note that this can always be done (e.g., in the extreme case, attach a control agent to each plant agent and assign the neighbour of $X_{i}$ to be its target).

Step 2: Ignoring all disturbances, derive expressions for the control laws $u[k]$, in terms of $v[k]$, as indicated in (5.12) and (5.14).

Step 3: Find matrix $K=\left[\begin{array}{ll}K_{1} & K_{2}\end{array}\right]$ using $(5.26)$ and (5.27) so that the eigenvalues of $A_{1}^{*}-B^{*} K$ are all at the origin. Set $v[k]=-K_{1} \bar{x}[k]-K_{2} w[k]$.

Step 4: Substitute $v[k]$ into the $u[k]$ from Step 2.

Figure 5.8: Summary of the proposed disturbance rejection design algorithm.

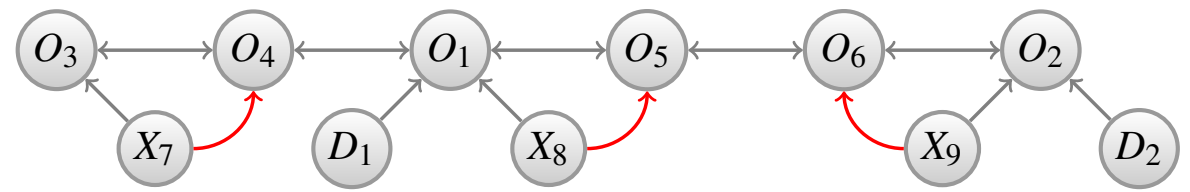

Figure 5.9: A six-agent queue plant with two disturbance agents.

$y_{i}[k]=x_{i}[k]$ for $i=1,4,5,6$, are

$$
\begin{aligned}
x_{1}[k+1] & =x_{1}[k]+x_{4}[k]+2 x_{5}[k]+u_{8}[k]+d_{1}[k] \\
x_{21}[k+1] & =-x_{22}[k]+u_{9}[k] \\
x_{22}[k+1] & =2 x_{21}[k]+d_{2}[k] \\
y_{2}[k] & =x_{21}[k]-x_{22}[k] \\
x_{31}[k+1] & =-2 x_{32}[k]+u_{7}[k] \\
x_{32}[k+1] & =x_{31}[k]-x_{32}[k]+x_{4}[k] \\
y_{3}[k] & =x_{31}[k]+x_{32}[k] \\
x_{4}[k+1] & =y_{3}[k]+x_{1}[k]+2 x_{4}[k] \\
x_{5}[k+1] & =x_{1}[k]+2 x_{6}[k] \\
x_{6}[k+1] & =2 y_{2}[k]+x_{5}[k]+x_{6}[k] .
\end{aligned}
$$

First, we verify that the conditions in Step 1 in Figure 5.8 are met. There are two control agents, $X_{8}$ and $X_{9}$, connected to the plant agents $O_{1}$ and $O_{2}$ where the two disturbance agents $D_{1}$ and $D_{2}$ are connected. This satisfies Lemma 1 . It is also easy to verify that the system meets the conditions in Theorem 3, as the control agents and respective targets are close to one another, and there are no nodes in common between different fastest path from control agents to their 
target agents. For Theorem 4, the structure of this system shows that both of the conditions hold, since each fastest path from $X_{i}$ to $T_{i}$ (for $7 \leq i \leq 9$ ) is unique, and each plant agent lies on some fastest path. Full targeting and growing analyses confirm that targeting and growing succeed. For Step 2, ignoring the disturbances, we force

$$
\begin{aligned}
& y_{4}[k+2]=v_{7}[k] \\
& y_{5}[k+2]=v_{8}[k] \\
& y_{6}[k+2]=v_{9}[k],
\end{aligned}
$$

as the propagation time to the target agents $O_{4}, O_{5}$ and $O_{6}$ are

$$
\begin{aligned}
& \Delta\left(X_{7}, O_{4}\right)=2 \\
& \Delta\left(X_{8}, O_{5}\right)=2 \\
& \Delta\left(X_{9}, O_{6}\right)=2
\end{aligned}
$$

This yields the control laws

$$
\begin{aligned}
u_{7}[k]= & v_{7}[k]-3 x_{1}[k]-3 x_{31}[k]+x_{32}[k] \\
& -6 x_{4}[k]+2 x_{5}[k]-u_{8}[k] \\
u_{8}[k]= & v_{8}[k]-x_{1}[k]-4 x_{21}[k]+4 x_{22}[k] \\
& -x_{4}[k]-4 x_{5}[k]-2 x_{6}[k] \\
u_{9}[k]= & \left(v_{9}[k]-x_{1}[k]+2 x_{21}[k]+4 x_{22}[k]\right. \\
& \left.-x_{5}[k]-3 x_{6}[k]\right) / 2 .
\end{aligned}
$$

For Step 3, we construct the augmented system (5.23)-(5.24):

$$
\begin{aligned}
x^{*}[k+1] & =A_{1}^{*} x^{*}[k]+B^{*}(v[k]+\tilde{d}[k]) \\
y^{\text {tar }}[k] & =C^{*} x^{*}[k]
\end{aligned}
$$

where

$$
\begin{aligned}
x^{*} & =\left[\begin{array}{c}
\bar{x}[k] \\
w[k]
\end{array}\right], \\
A_{1}^{*} & =\left[\begin{array}{ll}
\bar{A} & O \\
\bar{C} & I
\end{array}\right]
\end{aligned}
$$




$$
\begin{aligned}
= & {\left[\begin{array}{lllllllll}
0 & 1 & 0 & 0 & 0 & 0 & 0 & 0 & 0 \\
0 & 0 & 0 & 0 & 0 & 0 & 0 & 0 & 0 \\
0 & 0 & 0 & 1 & 0 & 0 & 0 & 0 & 0 \\
0 & 0 & 0 & 0 & 0 & 0 & 0 & 0 & 0 \\
0 & 0 & 0 & 0 & 0 & 1 & 0 & 0 & 0 \\
0 & 0 & 0 & 0 & 0 & 0 & 0 & 0 & 0 \\
1 & 0 & 0 & 0 & 0 & 0 & 1 & 0 & 0 \\
0 & 0 & 1 & 0 & 0 & 0 & 0 & 1 & 0 \\
0 & 0 & 0 & 0 & 1 & 0 & 0 & 0 & 1
\end{array}\right], } \\
B^{*} & {\left[\begin{array}{ll}
\bar{B} & \\
O
\end{array}\right] } \\
& {\left[\begin{array}{lll}
0 & 0 & 0 \\
1 & 0 & 0 \\
0 & 0 & 0 \\
0 & 1 & 0 \\
0 & 0 & 0 \\
0 & 0 & 1 \\
0 & 0 & 0 \\
0 & 0 & 0 \\
0 & 0 & 0
\end{array}\right], }
\end{aligned}
$$

with

$$
\begin{gathered}
\bar{A}=\text { block } \operatorname{diag}\left\{A_{\Delta\left(X_{7}, O_{3}\right)}^{\prime}, A_{\Delta\left(X_{8}, O_{5}\right)}^{\prime}, A_{\Delta\left(X_{9}, O_{6}\right)}^{\prime}\right\} \\
\bar{B}=\text { block diag }\left\{B_{\Delta\left(X_{7}, O_{3}\right)}^{\prime}, B_{\Delta\left(X_{8}, O_{5}\right)}^{\prime}, B_{\Delta\left(X_{9}, O_{6}\right)}^{\prime}\right\} \\
\bar{C}=\text { block diag }\left\{C_{\Delta\left(X_{7}, O_{3}\right)}^{\prime}, C_{\Delta\left(X_{8}, O_{5}\right)}^{\prime}, C_{\Delta\left(X_{9}, O_{6}\right)}^{\prime}\right\},
\end{gathered}
$$

where $\Delta\left(X_{7}, O_{3}\right)=\Delta\left(X_{8}, O_{5}\right)=\Delta\left(X_{9}, O_{6}\right)=2$ and

$$
A_{2}^{\prime}=\left[\begin{array}{ll}
0 & 1 \\
0 & 0
\end{array}\right], B_{2}^{\prime}=\left[\begin{array}{l}
0 \\
1
\end{array}\right], C_{2}^{\prime}=\left[\begin{array}{ll}
1 & 0
\end{array}\right] .
$$

We next determine $K_{1}$ and $K_{2}$ based on (5.26) and (5.27) to place all eigenvalues of $A_{1}^{*}-B^{*} K$ at the origin:

$$
K_{1}=\left[\begin{array}{llllll}
1 & 1 & 0 & 0 & 0 & 0 \\
0 & 0 & 1 & 1 & 0 & 0 \\
0 & 0 & 0 & 0 & 1 & 1
\end{array}\right], \quad K_{2}=\left[\begin{array}{lll}
1 & 0 & 0 \\
0 & 1 & 0 \\
0 & 0 & 1
\end{array}\right]
$$


Thus, the control law $v[k]$ in 5.25 is fully specified. For Step 4, substitute $v[k]$ into $5.64-(5.66)$ to complete the controller design as

$$
\begin{aligned}
u_{7}[k]= & v_{7}[k]-3 x_{1}[k]-3 x_{31}[k]+x_{32}[k] \\
& -6 x_{4}[k]+2 x_{5}[k]-u_{8}[k] \\
= & -\overline{x_{1}}[k]-\overline{x_{2}}[k]-w_{1}[k]-3 x_{1}[k]-3 x_{31}[k]+x_{32}[k] \\
& -6 x_{4}[k]+2 x_{5}[k]-u_{8}[k] \\
u_{8}[k]= & v_{8}[k]-x_{1}[k]-4 x_{21}[k]+4 x_{22}[k] \\
& -x_{4}[k]-4 x_{5}[k]-2 x_{6}[k] \\
= & -\overline{x_{3}}[k]-\overline{x_{4}}[k]-w_{2}[k]-x_{1}[k]-4 x_{21}[k]+4 x_{22}[k] \\
& -x_{4}[k]-4 x_{5}[k]-2 x_{6}[k] \\
u_{9}[k]= & \left(v_{9}[k]-x_{1}[k]+2 x_{21}[k]+4 x_{22}[k]\right. \\
& \left.-x_{5}[k]-3 x_{6}[k]\right) / 2 \\
= & \left(-\overline{x_{5}}[k]-\overline{x_{6}}[k]-w_{3}[k]-x_{1}[k]+2 x_{21}[k]+4 x_{22}[k]\right. \\
& \left.-x_{5}[k]-3 x_{6}[k]\right) / 2,
\end{aligned}
$$

where

$$
\bar{x}[k]=\left[v_{7}[k-2], v_{7}[k-1], v_{8}[k-2], v_{8}[k-1], v_{9}[k-2], v_{9}[k-1]\right]^{T} .
$$

Using the initial conditions and disturbances

$$
\begin{aligned}
& x[0]=\left[\begin{array}{llllll}
1 & x_{20} & x_{30} & 6 & 7 & 8
\end{array}\right]^{T}, \text { where } x_{20} \triangleq\left[\begin{array}{ll}
2 & 3
\end{array}\right], x_{30} \triangleq\left[\begin{array}{ll}
4 & 5
\end{array}\right] \\
& \bar{x}[0]=\left[\begin{array}{llllll}
0 & 0 & 0 & 0 & 0 & 0
\end{array}\right]^{T} \\
& w[0]=\left[\begin{array}{lll}
0 & 0 & 0
\end{array}\right]^{T} \\
& d_{1}[k]=d_{2}[k]=1(\text { for } k \geq 0) \text {, }
\end{aligned}
$$

simulations (see Figure 5.10) show that the control laws successfully drive the outputs of all agents to zero. Matlab code for the simulation is given in Appendix D. 

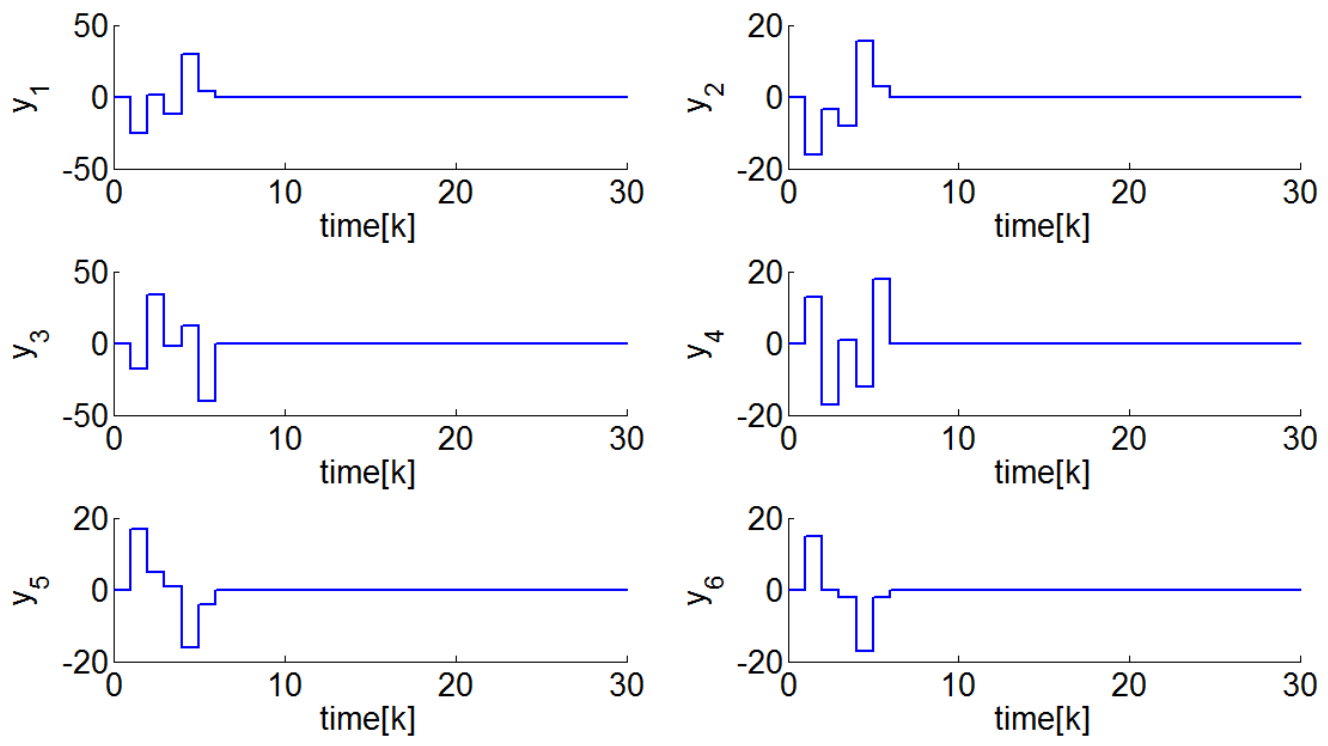

Figure 5.10: Simulation results for the six-agent queue system. 


\section{Chapter 6}

\section{Examples of Various Linear Multi-agent Systems}

In the last chapter we demonstrated, using a queue and some grid systems, the effectiveness of the proposed control strategy. In this chapter, several additional examples of linear multi-agent systems with different structures are given to further illustrate the design algorithm in Figure 5.8. We consider networks with the following graph structures: a ring, a spider, a grid, a wheel, complete graphs, and null graphs. For simplicity, we assume that all the links between plant agents are bidirectional, and that the propagation time from each agent to any neighbour agent is one time step, i.e, $\delta_{i j}=1$ and $\delta_{i}^{d}=1$. For the dynamical equations in this chapter, we also assume for simplicity that $y_{i}[k]=x_{i}[k]$ (for $1 \leq i \leq n$ ). We present the control algorithm for each example with the minimum number of control agents. Notice that in this chapter, we violate our earlier notational requirement that $D_{i}$ needs to be connected to plant agent $O_{i}$; the control algorithm remains the same with obvious notational changes. By the end of this chapter, we will have provided substantial evidence that the proposed control algorithm works well for the disturbance rejection problem for linear multi-agent systems.

\subsection{Ring Structure}

Consider the ring-structured system in Figure 6.1. This ring system has six plant agents and two disturbance agents. The dynamics of the ring system are as follows:

$$
\begin{aligned}
x_{1}[k+1] & =x_{2}[k]+x_{6}[k]+u_{7}[k]+d_{1}[k] \\
x_{2}[k+1] & =x_{1}[k]
\end{aligned}
$$




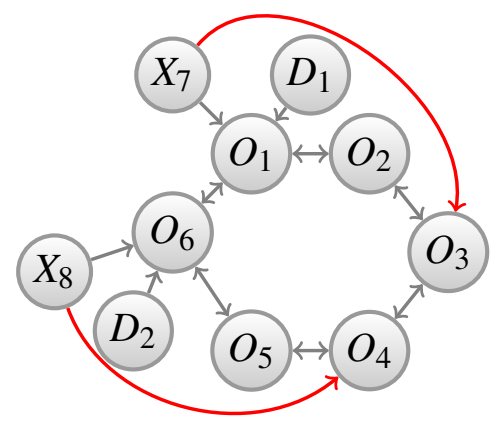

Figure 6.1: The ring system with two disturbance agents.

$$
\begin{aligned}
x_{3}[k+1] & =x_{2}[k]+x_{4}[k] \\
x_{4}[k+1] & =x_{3}[k]+x_{5}[k] \\
x_{5}[k+1] & =x_{6}[k] \\
x_{6}[k+1] & =x_{1}[k]+x_{5}[k]+u_{8}[k]+d_{2}[k] .
\end{aligned}
$$

According to the algorithm in Figure 5.8, the first step is to locate the control agents. Based on Lemma 1, two control agents $X_{1}$ and $X_{2}$ are put right beside agents $D_{1}$ and $D_{2}$, connected to plant agents $O_{1}$ and $O_{6}$. We set $O_{3}$ as the target of $X_{7}$ and $O_{4}$ as the target of $X_{8}$. The reader can verify that both targeting and growing succeed.

For Step 2, we ignore the disturbances and derive formulas for the control laws. From the network structure, we know that

$$
\begin{aligned}
& \Delta\left(X_{7}, O_{3}\right)=3 \\
& \Delta\left(X_{8}, O_{4}\right)=3 .
\end{aligned}
$$

Then we get

$$
\begin{aligned}
x_{3}[k+3] & =2 x_{2}[k]+x_{4}[k]+2 x_{6}[k]+u_{7}[k] \\
& =v_{7}[k] \\
x_{4}[k+3] & =2 x_{1}[k]+2 x_{5}[k]+x_{3}[k]+u_{8}[k] \\
& =v_{8}[k] .
\end{aligned}
$$

From 6.7) and (6.8), the control laws are

$$
\begin{aligned}
& u_{7}[k]=v_{7}[k]-2 x_{2}[k]-x_{4}[k]-2 x_{6}[k] \\
& u_{8}[k]=v_{8}[k]-2 x_{1}[k]-2 x_{5}[k]-x_{3}[k] .
\end{aligned}
$$


In Step 3 we find matrix $K=\left[\begin{array}{ll}K_{1} & K_{2}\end{array}\right]$ so that all the eigenvalues of $A_{1}^{*}-B^{*} K$ are at the origin. Using

$$
\begin{aligned}
\bar{A} & =\text { block } \operatorname{diag}\left\{A_{\Delta\left(X_{7}, T_{7}\right)}^{\prime}, A_{\Delta\left(X_{8}, T_{8}\right)}^{\prime}\right\} \\
\bar{B} & =\text { block } \operatorname{diag}\left\{B_{\Delta\left(X_{7}, T_{7}\right)}^{\prime}, B_{\Delta\left(X_{8}, T_{8}\right)}^{\prime}\right\},
\end{aligned}
$$

we determine

$$
\begin{aligned}
A_{1}^{*}= & {\left[\begin{array}{llllll}
0 & 1 & 0 & 0 & 0 & 0 \\
0 & 0 & 1 & 0 & 0 & 0 \\
0 & 0 & 0 & 0 & 0 & 0 \\
0 & 0 & 0 & 0 & 1 & 0 \\
0 & 0 & 0 & 0 & 0 & 1 \\
0 & 0 & 0 & 0 & 0 & 0
\end{array}\right] \in R^{r \times r}, } \\
B^{*}= & {\left[\begin{array}{ll}
0 & 0 \\
0 & 0 \\
1 & 0 \\
0 & 0 \\
0 & 0 \\
0 & 1
\end{array}\right] \in R^{r \times 1} . }
\end{aligned}
$$

Hence, using(5.26) and (5.27), we set

$$
K_{1}=\left[\begin{array}{llllll}
1 & 1 & 1 & 0 & 0 & 0 \\
0 & 0 & 0 & 1 & 1 & 1
\end{array}\right], K_{2}=\left[\begin{array}{ll}
1 & 0 \\
0 & 1
\end{array}\right] .
$$

The control law $v[k]=-K_{1} v[k]-K_{2} w[k]$ is now fully specified. Substitute $v[k]$ into 6.9 and 6.10) to obtain

$$
\begin{aligned}
u_{7}[k] & =v_{7}[k]-2 x_{2}[k]-x_{4}[k]-2 x_{6}[k] \\
& =-\overline{x_{1}}[k]-\overline{x_{2}}[k]-\overline{x_{3}}[k]-w_{1}[k]-2 x_{2}[k]-x_{4}[k]-2 x_{6}[k] \\
u_{8}[k] & =v_{8}[k]-2 x_{1}[k]-2 x_{5}[k]-x_{3}[k] \\
& =-\overline{x_{4}}[k]-\overline{x_{5}}[k]-\overline{x_{6}}[k]-w_{2}[k]-2 x_{1}[k]-2 x_{5}[k]-x_{3}[k],
\end{aligned}
$$

where

$$
\bar{x}[k]=\left[v_{7}[k-3], v_{7}[k-2], v_{7}[k-1], v_{8}[k-3], v_{8}[k-2], v_{8}[k-1]\right]^{T} .
$$

Set the initial conditions and disturbances as follows:

$$
\begin{aligned}
x[k] & =\left[\begin{array}{llllll}
1 & 2 & 3 & 4 & 5 & 6
\end{array}\right]^{T} \\
\bar{x}[k] & =\left[\begin{array}{llllll}
0 & 0 & 0 & 0 & 0 & 0
\end{array}\right]^{T} \\
w[k] & =\left[\begin{array}{lll}
0 & 0
\end{array}\right]^{T} \\
d_{1}[k] & =1(\text { for } k \geq 0) \\
d_{2}[k] & =1(\text { for } k \geq 0) .
\end{aligned}
$$



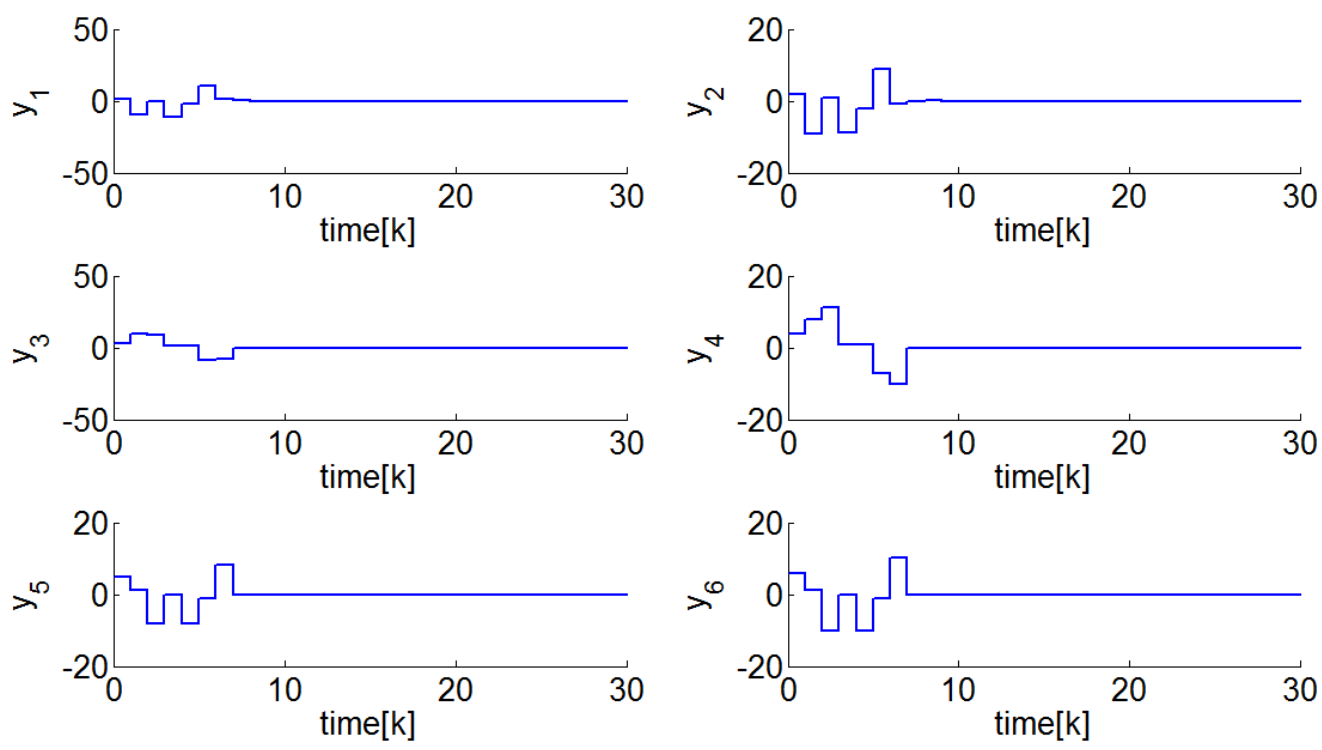

Figure 6.2: Simulation result for the ring structure system example.

The simulation results in Figure 6.2 show that the algorithm works. It rejects all the disturbances and zeros all the output signals of the plant agents in seven time steps.

\subsection{Spider Structure}

In this section, we study two spider structure systems. Consider the first spider structure system in Figure 6.3. This system has six plant agents and two disturbance agents. The dynamics of this spider system are as the follows:

$$
\begin{aligned}
& x_{1}[k+1]=x_{2}[k]+x_{4}[k]+x_{5}[k]+x_{6}[k] \\
& x_{2}[k+1]=x_{1}[k]+x_{2}[k]+x_{3}[k] \\
& x_{3}[k+1]=2 x_{2}[k] \\
& x_{4}[k+1]=x_{1}[k]+x_{4}[k]+u_{9}[k]+d_{1}[k] \\
& x_{5}[k+1]=x_{1}[k]+x_{5}[k]+u_{8}[k] \\
& x_{6}[k+1]=x_{1}[k]+x_{6}[k]+u_{7}[k]+d_{2}[k] .
\end{aligned}
$$

From Lemma 1, we know that the system needs at least two control agents, attached to the plant agents to which the two disturbance agents $D_{1}$ and $D_{2}$ are attached. But for targeting and growing to succeed, additional control agents are needed. There are four branches in this system. Using 


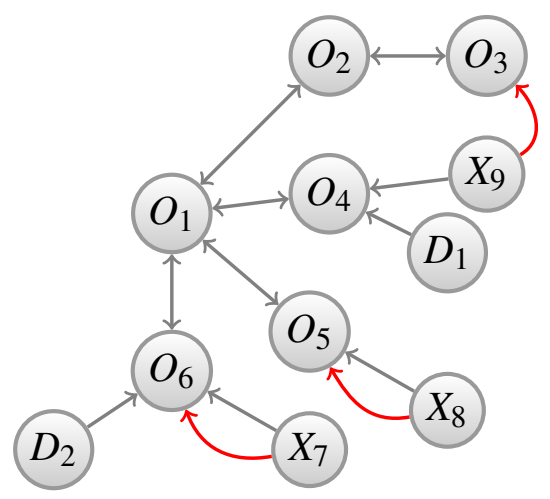

Figure 6.3: The spider system with six plant agents and two disturbance agents.

Theorem 3 and 4, we conclude that targeting and growing analyses succeed only if at least three control agents are introduced to the system. We set the control agents and assign their targets as shown in Figure 6.3. Targeting and growing analyses succeed for this arrangement.

Calculate the propagation time from $X_{i}$ to $T_{i}$ (for $7 \leq i \leq 9$ ):

$$
\begin{aligned}
& \Delta\left(X_{7}, O_{6}\right)=1 \\
& \Delta\left(X_{8}, O_{5}\right)=1 \\
& \Delta\left(X_{9}, O_{3}\right)=4
\end{aligned}
$$

Hence,

$$
\begin{aligned}
x_{6}[k+1]= & x_{1}[k]+x_{6}[k]+u_{7}[k] \\
= & v_{7}[k] \\
x_{5}[k+1]= & x_{1}[k]+x_{5}[k]+u_{8}[k] \\
= & v_{8}[k] \\
x_{3}[k+4]= & 2 x_{2}[k+3] \\
= & 2 x_{1}[k+2]+2 x_{2}[k+2]+2 x_{3}[k+2] \\
= & 2 x_{1}[k+1]+8 x_{2}[k+1]+2 x_{3}[k+1]+2 x_{4}[k+1]+2 x_{5}[k+1]+2 x_{6}[k+1] \\
= & 14 x_{1}[k]+14 x_{2}[k]+8 x_{3}[k]+4 x_{4}[k]+4 x_{5}[k] \\
& +4 x_{6}[k]+2 u_{7}[k]+2 u_{8}[k]+2 u_{9}[k] \\
= & v_{9}[k] .
\end{aligned}
$$

The formulas of the control laws are derived from 6.17) 6.19 as

$$
u_{7}[k]=v_{7}[k]-x_{1}[k]-x_{6}[k]
$$




$$
\begin{aligned}
u_{8}[k]= & v_{8}[k]-x_{1}[k]-x_{5}[k] \\
u_{9}[k]= & 0.5 v_{9}[k]-7 x_{1}[k]-7 x_{2}[k]-4 x_{3}[k]-2 x_{4}[k] \\
& -2 x_{5}[k]-2 x_{6}[k]-u_{7}[k]-u_{8}[k] .
\end{aligned}
$$

The next step is to find the $K$ matrix and the control laws for $v[k]$. As we know the propagation time from all the control agents to their targets, we determine the $K_{1}$ and $K_{2}$ matrices to be

$$
K_{1}=\left[\begin{array}{llllll}
1 & 0 & 0 & 0 & 0 & 0 \\
0 & 1 & 0 & 0 & 0 & 0 \\
0 & 0 & 1 & 1 & 1 & 1
\end{array}\right], K_{2}=\left[\begin{array}{lll}
1 & 0 & 0 \\
0 & 1 & 0 \\
0 & 0 & 1
\end{array}\right]
$$

and the $v[k]$ control laws to be

$$
\begin{aligned}
& v_{7}[k]=-\bar{x}_{1}[k]-w_{1}[k] \\
& v_{8}[k]=-\bar{x}_{2}[k]-w_{2}[k] \\
& v_{9}[k]=-\bar{x}_{3}[k]-\bar{x}_{4}[k]-\bar{x}_{5}[k]-\bar{x}_{6}[k]-w_{3}[k] .
\end{aligned}
$$

The final control laws of $u[k]$ are

$$
\begin{aligned}
u_{7}[k]= & v_{7}[k]-x_{1}[k]-x_{6}[k] \\
= & -\bar{x}_{1}[k]-w_{1}[k]-x_{1}[k]-x_{6}[k] \\
u_{8}[k]= & v_{8}[k]-x_{1}[k]-x_{5}[k] \\
= & -\bar{x}_{2}[k]-w_{2}[k]-x_{1}[k]-x_{5}[k] \\
u_{9}[k]= & 0.5 v_{9}[k]-7 x_{1}[k]-7 x_{2}[k]-4 x_{3}[k]-2 x_{4}[k]-2 x_{5}[k]-2 x_{6}[k]-u_{7}[k]-u_{8}[k] \\
= & 0.5\left(-\bar{x}_{3}[k]-\bar{x}_{4}[k]-\bar{x}_{5}[k]-\bar{x}_{6}[k]-w_{3}[k]\right) \\
& -7 x_{1}[k]-7 x_{2}[k]-4 x_{3}[k]-2 x_{4}[k]-2 x_{5}[k]-2 x_{6}[k]-u_{7}[k]-u_{8}[k],
\end{aligned}
$$

where

$$
\bar{x}[k]=\left[v_{7}[k-1], v_{8}[k-1], v_{9}[k-4], v_{9}[k-3], v_{9}[k-2], v_{9}[k-1]\right]^{T} .
$$

To run a simulation, we set the initial conditions and disturbances as

$$
\begin{aligned}
x[k] & =\left[\begin{array}{llllll}
1 & 2 & 3 & 4 & 5 & 6
\end{array}\right]^{T} \\
\bar{x}[k] & =\left[\begin{array}{llllll}
0 & 0 & 0 & 0 & 0 & 0
\end{array}\right]^{T} \\
w[k] & =\left[\begin{array}{lll}
0 & 0 & 0
\end{array}\right]^{T} \\
d_{1}[k] & =1(\text { for } k \geq 0) \\
d_{2}[k] & =1(\text { for } k \geq 0) .
\end{aligned}
$$

From the simulation result in Figure 6.4, we conclude that the proposed algorithm works perfectly in rejecting the disturbances and zeroing all plant agents. 

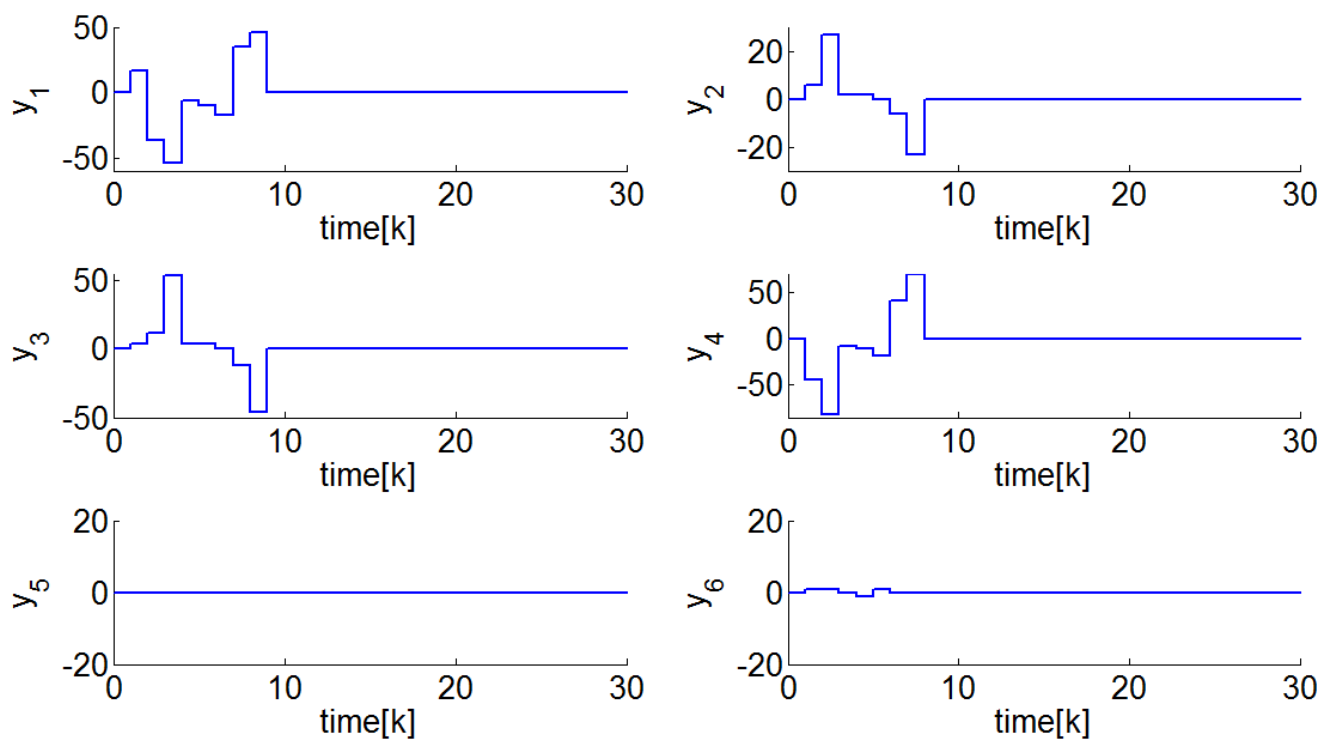

Figure 6.4: Simulation result for the first spider structure system example.

Now consider the second spider structure system in Figure 6.5. The structure of this system looks like the one in Figure 6.3. They both have two disturbances, three control agents and four branches. Even the way we assign targets for the control agents is similar. The only difference is that this system has one more plant agent.

The dynamics of the second spider system are

$$
\begin{aligned}
& x_{1}[k+1]=x_{2}[k]+x_{4}[k]+x_{6}[k]+x_{7}[k] \\
& x_{2}[k+1]=x_{1}[k]+0.5 x_{3}[k] \\
& x_{3}[k+1]=x_{2}[k]+x_{3}[k] \\
& x_{4}[k+1]=x_{1}[k]+0.5 x_{4}[k]+x_{5}[k] \\
& x_{5}[k+1]=0.5 x_{4}[k]+u_{10}[k]+d_{2}[k] \\
& x_{6}[k+1]=x_{1}[k]+0.5 x_{6}[k]+u_{9}[k] \\
& x_{7}[k+1]=0.5 x_{1}[k]+u_{8}[k]+d_{1}[k] .
\end{aligned}
$$

The propagation time from the three control agents to their respective target agents are

$$
\begin{aligned}
\Delta\left(X_{8}, O_{7}\right) & =1 \\
\Delta\left(X_{9}, O_{6}\right) & =1 \\
\Delta\left(X_{10}, O_{3}\right) & =5,
\end{aligned}
$$




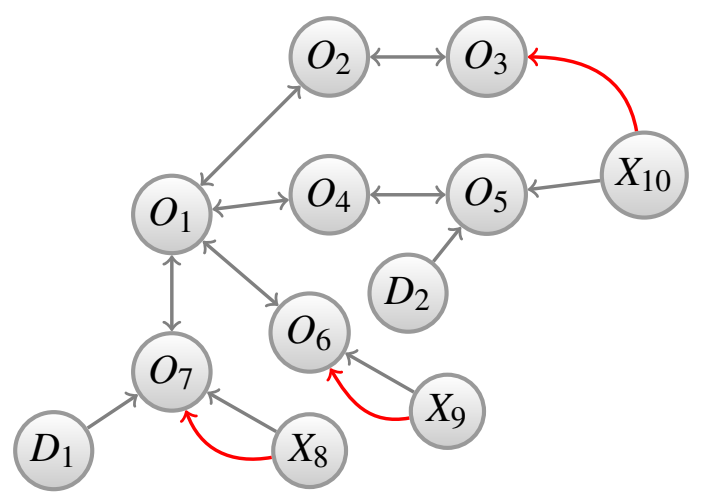

Figure 6.5: The spider system with seven plant agents and two disturbance agents.

so we determine that

$$
\begin{aligned}
x_{6}[k+1]= & 0.5 x_{1}[k]+u_{8}[k] \\
= & v_{8}[k] \\
x_{5}[k+1]= & x_{1}[k]+0.5 x_{6}[k]+u_{9}[k] \\
= & v_{9}[k] \\
x_{3}[k+5]= & x_{2}[k+4]+x_{3}[k+4] \\
= & x_{1}[k+3]+x_{2}[k+3]+1.5 x_{3}[k+3] \\
= & x_{1}[k+2]+2.5 x_{2}[k+2]+2 x_{3}[k+2]+x_{4}[k+2]+x_{6}[k+2]+x_{7}[k+2] \\
= & 5 x_{1}[k+1]+3 x_{2}[k+1]+3.25 x_{3}[k+1]+1.5 x_{4}[k+1]+x_{5}[k+1] \\
& +1.5 x_{6}[k+1]+x_{7}[k+1]+u_{8}[k+1]+u_{9}[k+1] \\
= & 6 x_{1}[k]+6.75 x_{2}[k]+4.75 x_{3}[k]+4.75 x_{4}[k]+1.5 x_{5}[k] \\
& +4 x_{6}[k]+3.5 x_{7}[k]+u_{8}[k]+u_{9}[k]+u_{10}[k] \\
= & v_{10}[k] .
\end{aligned}
$$

Thus the formulas of the control laws can be derived from 6.33$)-(6.36)$ as

$$
\begin{aligned}
u_{8}[k]= & v_{8}[k]-0.5 x_{1}[k] \\
u_{9}[k]= & v_{9}[k]-x_{1}[k]-0.5 x_{6}[k] \\
u_{10}[k]= & v_{10}[k]-6 x_{1}[k]-6.75 x_{2}[k]-4.75 x_{3}[k]-4.75 x_{4}[k]-1.5 x_{5}[k] \\
& -4 x_{6}[k]-3.5 x_{7}[k]-u_{8}[k]-u_{9}[k] .
\end{aligned}
$$

Notice that terms $u_{8}[k+1]$ and $u_{9}[k+1]$ appear in 6.35 . However, we want $y^{\operatorname{tar}}[k]$ to be ex- 
pressed as (for $n+1 \leq i \leq n+m)$

$$
\begin{gathered}
y_{i}^{\text {tar }}\left[k+\Delta_{i}\right]=\sum_{q \neq i}\left(\alpha_{u}^{q i} u_{q}[k]+\alpha_{d}^{q i} d_{q}[k]\right)+\alpha_{1}^{i} x_{1}[k]+\alpha_{2}^{i} x_{2}[k]+ \\
\cdots+\alpha_{n}^{i} x_{n}[k]+\alpha_{u}^{i} u_{i}[k]+\alpha_{d}^{i} d_{i}[k]
\end{gathered}
$$

To this end, we substitute the expressions

$$
\begin{aligned}
& u_{8}[k+1]=-0.5 x_{1}[k+1] \\
& u_{9}[k+1]=-x_{1}[k+1]-0.5 x_{6}[k+1],
\end{aligned}
$$

into 6.35) at time step $k+1$, where we set $v_{8}[k+1]=0$ and $v_{9}[k+1]=0$ in (6.37) and (6.38). By substituting 6.40) and (6.41) into 6.35, we get the formula of $y_{3}^{\operatorname{tar}}[k+5]$.

Next, determine the $K_{1}$ and $K_{2}$ matrices

$$
K_{1}=\left[\begin{array}{lllllll}
1 & 0 & 0 & 0 & 0 & 0 & 0 \\
0 & 1 & 0 & 0 & 0 & 0 & 0 \\
0 & 0 & 1 & 1 & 1 & 1 & 1
\end{array}\right], K_{2}=\left[\begin{array}{lll}
1 & 0 & 0 \\
0 & 1 & 0 \\
0 & 0 & 1
\end{array}\right],
$$

and the $v[k]$ control laws

$$
\begin{aligned}
v_{8}[k] & =-\bar{x}_{1}[k]-w_{1}[k] \\
v_{9}[k] & =-\bar{x}_{2}[k]-w_{2}[k] \\
v_{10}[k] & =-\bar{x}_{3}[k]-\bar{x}_{4}[k]-\bar{x}_{5}[k]-\bar{x}_{6}[k]-\bar{x}_{7}[k]-w_{3}[k] .
\end{aligned}
$$

The final control laws for $u[k]$ are

$$
\begin{aligned}
u_{8}[k]= & v_{8}[k]-0.5 x_{1}[k] \\
= & -\bar{x}_{1}[k]-w_{1}[k]-0.5 x_{1}[k] \\
u_{9}[k]= & v_{9}[k]-x_{1}[k]-0.5 x_{6}[k] \\
= & -\bar{x}_{2}[k]-w_{2}[k]-x_{1}[k]-0.5 x_{6}[k] \\
u_{10}[k]= & v_{10}[k]-6 x_{1}[k]-6.75 x_{2}[k]-4.75 x_{3}[k]-4.75 x_{4}[k]-1.5 x_{5}[k] \\
& -4 x_{6}[k]-3.5 x_{7}[k]-u_{8}[k]-u_{9}[k] \\
= & -\bar{x}_{3}[k]-\bar{x}_{4}[k]-\bar{x}_{5}[k]-\bar{x}_{6}[k]-\bar{x}_{7}[k]-w_{3}[k] \\
& -6 x_{1}[k]-6.75 x_{2}[k]-4.75 x_{3}[k]-4.75 x_{4}[k]-1.5 x_{5}[k] \\
& -4 x_{6}[k]-3.5 x_{7}[k]-u_{8}[k]-u_{9}[k],
\end{aligned}
$$

where

$$
\bar{x}[k]=\left[v_{8}[k-1], v_{9}[k-1], v_{10}[k-4], v_{10}[k-3], v_{10}[k-2], v_{10}[k-1]\right]^{T} .
$$



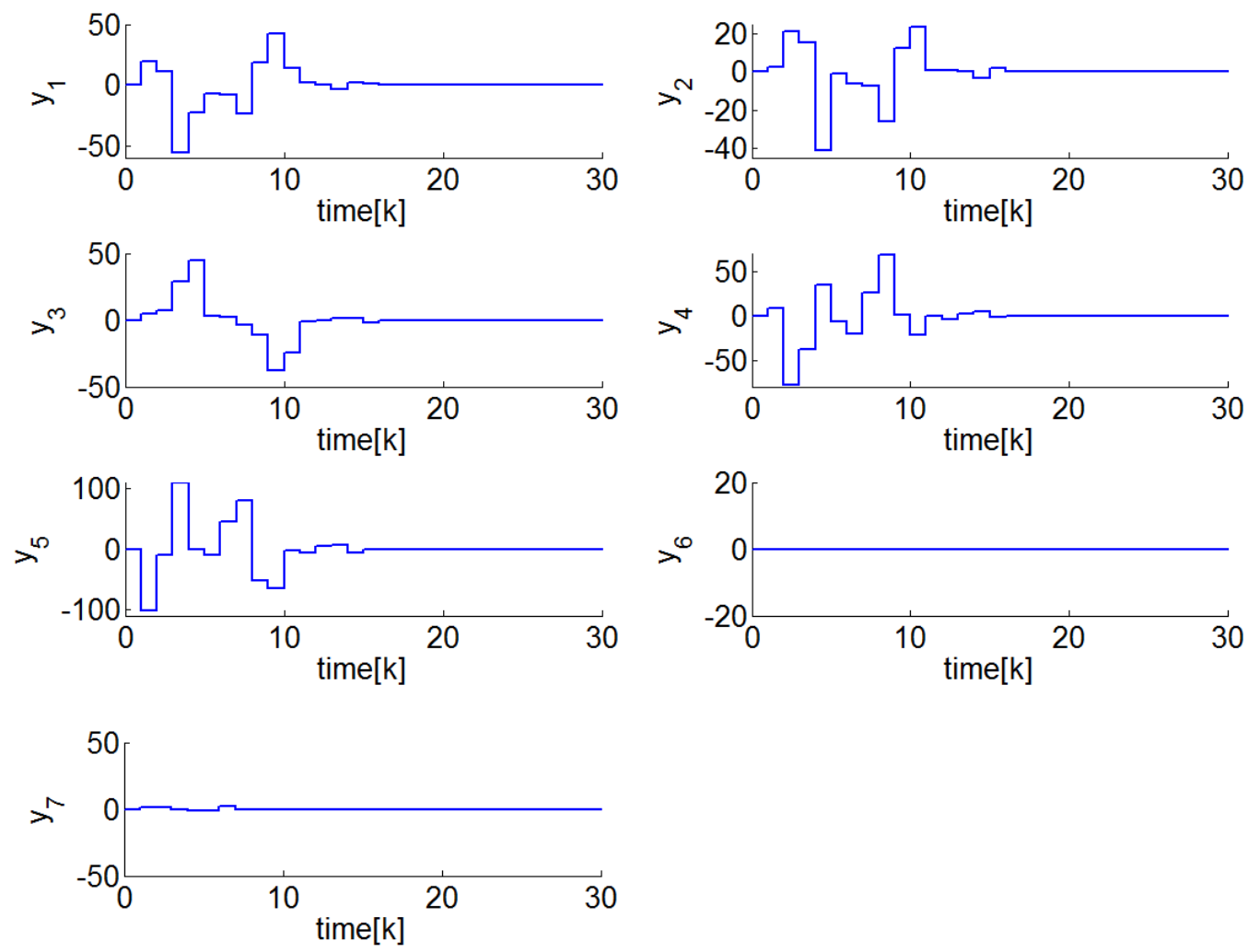

Figure 6.6: Simulation result for the second spider structure system example.

Set the initial conditions and disturbances as

$$
\begin{aligned}
x[k] & =\left[\begin{array}{lllllll}
1 & 2 & 3 & 4 & 5 & 6 & 7
\end{array}\right]^{T} \\
\bar{x}[k] & =\left[\begin{array}{llllll}
0 & 0 & 0 & 0 & 0 & 0
\end{array}\right]^{T} \\
w[k] & =\left[\begin{array}{lll}
0 & 0 & 0
\end{array}\right]^{T} \\
d_{1}[k] & =1(\text { for } k \geq 0) \\
d_{2}[k] & =1(\text { for } k \geq 0) .
\end{aligned}
$$

The simulation result in Figure 6.6 shows that disturbance rejection is obtained again, although derivation of the control laws for the second spider example is trickier than for the first spider example. 


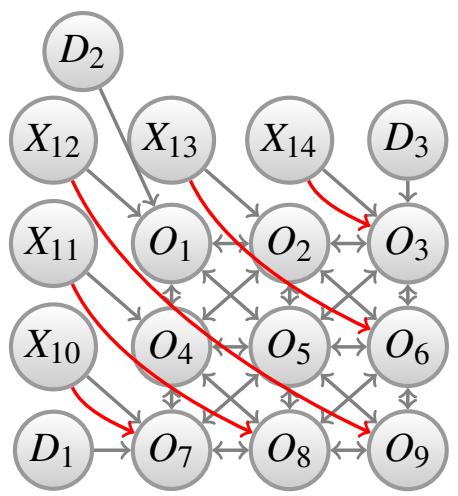

Figure 6.7: The grid structure system with nine plant agents and three disturbance agents.

\subsection{Grid Structure}

In this section, we consider an example of a grid structure with nine agents where additional diagonal edges are included between plant agents. As shown in Figure 6.7, there are three disturbances connected to the grid structure system. The dynamics are taken to be

$$
\begin{aligned}
x_{1}[k+1] & =x_{2}[k]+x_{4}[k]+x_{5}[k]+u_{12}[k]+d_{2}[k] \\
x_{2}[k+1] & =x_{1}[k]+x_{3}[k]+x_{4}[k]+x_{5}[k]+x_{6}[k]+u_{13}[k] \\
x_{3}[k+1] & =x_{2}[k]+x_{5}[k]+x_{6}[k]+u_{14}[k]+d_{3}[k] \\
x_{4}[k+1] & =x_{1}[k]+x_{2}[k]+x_{5}[k]+x_{7}[k]+x_{8}[k]+u_{11}[k] \\
x_{5}[k+1] & =x_{1}[k]+x_{2}[k]+x_{3}[k]+x_{4}[k]+x_{6}[k]+x_{7}[k]+x_{8}[k]+x_{9}[k] \\
x_{6}[k+1] & =x_{2}[k]+x_{3}[k]+x_{5}[k]+x_{8}[k]+x_{9}[k] \\
x_{7}[k+1] & =x_{4}[k]+x_{5}[k]+x_{8}[k]+u_{10}[k]+d_{1}[k] \\
x_{8}[k+1] & =x_{4}[k]+x_{5}[k]+x_{6}[k]+x_{7}[k]+x_{9}[k] \\
x_{9}[k+1] & =x_{5}[k]+x_{6}[k]+x_{8}[k] .
\end{aligned}
$$

Based on Lemma 1, a control agent must be placed next to each of the three disturbance agents, so we place $X_{10}$ connected to $O_{7}, X_{12}$ connected to $O_{1}$, and $X_{14}$ connected to $O_{3}$. However, using just these three control agents, targets cannot be chosen such that each of the nine plant agents lies on one of the fastest paths from some control agent to its target. To satisfy the conditions of Theorems 3 and 4, we add two more control agents, $X_{11}$ and $X_{13}$, as indicated in Figure 6.7. For this arrangement, targeting and growing analyses succeed.

The propagation time from the control agents to their target agents are

$$
\Delta\left(X_{10}, O_{7}\right)=1
$$




$$
\begin{aligned}
& \Delta\left(X_{11}, O_{8}\right)=2 \\
& \Delta\left(X_{12}, O_{9}\right)=3 \\
& \Delta\left(X_{13}, O_{6}\right)=2 \\
& \Delta\left(X_{14}, O_{3}\right)=1 .
\end{aligned}
$$

To derive the expressions for the control laws $u[k]$, we first determine

$$
\begin{aligned}
x_{7}[k+1]= & x_{4}[k]+x_{5}[k]+x_{8}[k]+u_{10}[k] \\
= & v_{10}[k] \\
x_{8}[k+2]= & x_{4}[k+1]+x_{5}[k+1]+x_{6}[k+1]+x_{7}[k+1]+x_{9}[k+1] \\
= & 2 x_{1}[k]+3 x_{2}[k]+2 x_{3}[k]+2 x_{4}[k]+4 x_{5}[k]+2 x_{6}[k] \\
& +2 x_{7}[k]+5 x_{8}[k]+2 x_{9}[k]+u_{10}[k]+u_{11}[k] \\
= & v_{11}[k] \\
x_{9}[k+3]= & x_{5}[k+2]+x_{6}[k+2]+x_{8}[k+2] \\
= & x_{1}[k+1]+2 x_{2}[k+1]+2 x_{3}[k+1]+2 x_{4}[k+1]+2 x_{5}[k+1] \\
& +2 x_{6}[k+1]+2 x_{7}[k+1]+2 x_{8}[k+1]+3 x_{9}[k+1] \\
= & 6 x_{1}[k]+9 x_{2}[k]+6 x_{3}[k]+9 x_{4}[k]+16 x_{5}[k]+11 x_{6}[k]+6 x_{7}[k] \\
& +11 x_{8}[k]+6 x_{9}[k]+2 u_{10}[k]+2 u_{11}[k]+u_{12}[k]+2 u_{13}[k]+2 u_{14}[k] \\
= & v_{12}[k] \\
x_{6}[k+2]= & x_{2}[k+1]+x_{3}[k+1]+x_{5}[k+1]+x_{8}[k+1]+x_{9}[k+1] \\
= & 2 x_{1}[k]+2 x_{2}[k]+2 x_{3}[k]+3 x_{4}[k]+4 x_{5}[k]+5 x_{6}[k] \\
& +2 x_{7}[k]+2 x_{8}[k]+2 x_{9}[k]+u_{13}[k]+u_{14}[k] \\
= & v_{13}[k] \\
= & x_{2}[k]+x_{5}[k]+x_{6}[k]+u_{14}[k] \\
= & v_{14}[k],
\end{aligned}
$$

from which we derive that

$$
\begin{aligned}
u_{10}[k]= & v_{10}[k]-x_{4}[k]-x_{5}[k]-x_{8}[k] \\
u_{11}[k]= & v_{11}[k]-2 x_{1}[k]-3 x_{2}[k]-2 x_{3}[k]-2 x_{4}[k]-4 x_{5}[k] \\
& -2 x_{6}[k]-2 x_{7}[k]-5 x_{8}[k]-2 x_{9}[k]-u_{10}[k] \\
u_{12}[k]= & v_{12}[k]-6 x_{1}[k]-9 x_{2}[k]-6 x_{3}[k]-9 x_{4}[k]-16 x_{5}[k]-11 x_{6}[k] \\
& -6 x_{7}[k]-11 x_{8}[k]-6 x_{9}[k]-2 u_{10}[k]-2 u_{11}[k]-2 u_{13}[k]-2 u_{14}[k] \\
u_{13}[k]= & v_{13}[k]-2 x_{1}[k]-2 x_{2}[k]-2 x_{3}[k]-3 x_{4}[k]-4 x_{5}[k]-5 x_{6}[k] \\
& -2 x_{7}[k]-2 x_{8}[k]-2 x_{9}[k]-u_{14}[k] \\
u_{14}[k]= & v_{14}[k]-x_{2}[k]-x_{5}[k]-x_{6}[k] .
\end{aligned}
$$


Using the propagation times for the fastest paths from $X_{i}$ to $T_{i}$ (for $10 \leq i \leq 14$ ), we can determine the matrices $K_{1}$ and $K_{2}$, following (5.26) and (5.27), to be

$$
K_{1}=\left[\begin{array}{lllllllll}
1 & 0 & 0 & 0 & 0 & 0 & 0 & 0 & 0 \\
0 & 1 & 1 & 0 & 0 & 0 & 0 & 0 & 0 \\
0 & 0 & 0 & 1 & 1 & 1 & 0 & 0 & 0 \\
0 & 0 & 0 & 0 & 0 & 0 & 1 & 1 & 0 \\
0 & 0 & 0 & 0 & 0 & 0 & 0 & 0 & 1
\end{array}\right], K_{2}=\left[\begin{array}{lllll}
1 & 0 & 0 & 0 & 0 \\
0 & 1 & 0 & 0 & 0 \\
0 & 0 & 1 & 0 & 0 \\
0 & 0 & 0 & 1 & 0 \\
0 & 0 & 0 & 0 & 1
\end{array}\right]
$$

The control laws $v[k]$ are

$$
\begin{aligned}
& v_{10}[k]=-\bar{x}_{1}[k]-w_{1}[k] \\
& v_{11}[k]=-\bar{x}_{2}[k]-\bar{x}_{3}[k]-w_{2}[k] \\
& v_{12}[k]=-\bar{x}_{4}[k]-\bar{x}_{5}[k]-\bar{x}_{6}[k]-w_{3}[k] \\
& v_{13}[k]=-\bar{x}_{7}[k]-\bar{x}_{8}[k]-w_{4}[k] \\
& v_{14}[k]=-\bar{x}_{9}[k]-w_{5}[k] .
\end{aligned}
$$

Substitute $v[k]$ into $6.59-6.63$

$$
\begin{aligned}
u_{10}[k]= & v_{10}[k]-x_{4}[k]-x_{5}[k]-x_{8}[k] \\
= & -\bar{x}_{1}[k]-w_{1}[k]-x_{4}[k]-x_{5}[k]-x_{8}[k] \\
u_{11}[k]= & v_{11}[k]-2 x_{1}[k]-3 x_{2}[k]-2 x_{3}[k]-2 x_{4}[k]-4 x_{5}[k] \\
& -2 x_{6}[k]-2 x_{7}[k]-5 x_{8}[k]-2 x_{9}[k]-u_{10}[k] \\
= & -\bar{x}_{2}[k]-\bar{x}_{3}[k]-w_{2}[k]-2 x_{1}[k]-3 x_{2}[k]-2 x_{3}[k] \\
& -2 x_{4}[k]-4 x_{5}[k]-2 x_{6}[k]-2 x_{7}[k]-5 x_{8}[k]-2 x_{9}[k]-u_{10}[k] \\
u_{12}[k]= & v_{12}[k]-6 x_{1}[k]-9 x_{2}[k]-6 x_{3}[k]-9 x_{4}[k]-16 x_{5}[k]-11 x_{6}[k] \\
& -6 x_{7}[k]-11 x_{8}[k]-6 x_{9}[k]-2 u_{10}[k]-2 u_{11}[k]-2 u_{13}[k]-2 u_{14}[k] \\
= & -\bar{x}_{4}[k]-\bar{x}_{5}[k]-\bar{x}_{6}[k]-w_{3}[k]-6 x_{1}[k]-9 x_{2}[k] \\
& -6 x_{3}[k]-9 x_{4}[k]-16 x_{5}[k]-11 x_{6}[k]-6 x_{7}[k]-11 x_{8}[k]-6 x_{9}[k] \\
& -2 u_{10}[k]-2 u_{11}[k]-2 u_{13}[k]-2 u_{14}[k] \\
u_{13}[k]= & v_{13}[k]-2 x_{1}[k]-2 x_{2}[k]-2 x_{3}[k]-3 x_{4}[k]-4 x_{5}[k]-5 x_{6}[k] \\
& -2 x_{7}[k]-2 x_{8}[k]-2 x_{9}[k]-u_{14}[k] \\
= & -\bar{x}_{7}[k]-\bar{x}_{8}[k]-w_{4}[k]-2 x_{1}[k]-2 x_{2}[k]-2 x_{3}[k]-3 x_{4}[k] \\
& -4 x_{5}[k]-5 x_{6}[k]-2 x_{7}[k]-2 x_{8}[k]-2 x_{9}[k]-u_{14}[k] \\
= & v_{14}[k]-x_{2}[k]-x_{5}[k]-x_{6}[k] \\
= & -\bar{x}_{9}[k]-w_{5}[k]-x_{2}[k]-x_{5}[k]-x_{6}[k], \\
u_{14}[k] &
\end{aligned}
$$



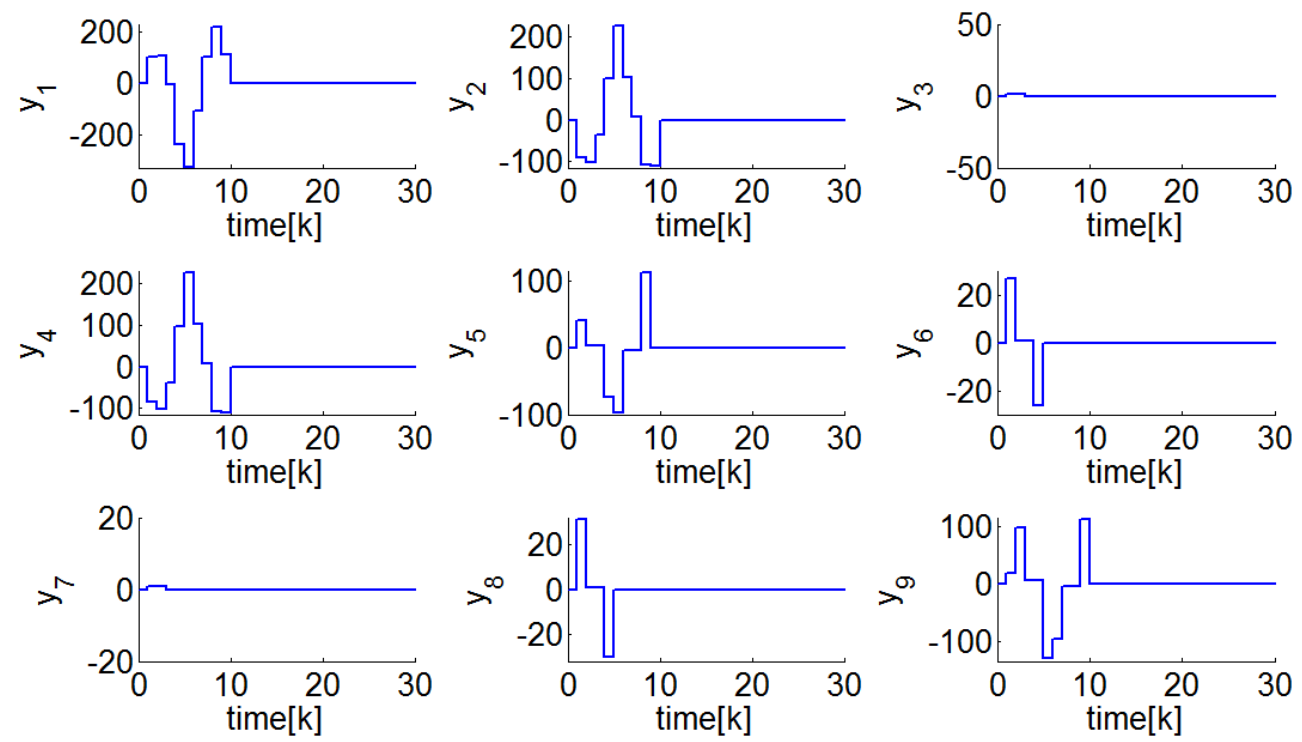

Figure 6.8: Simulation result for the grid structure system example.

where

$$
\begin{array}{r}
\bar{x}[k]=\left[v_{10}[k-1], v_{11}[k-2], v_{11}[k-1], v_{12}[k-3], v_{12}[k-2],\right. \\
\left.v_{12}[k-1], v_{13}[k-2], v_{13}[k-1], v_{14}[k-1]\right]^{T} .
\end{array}
$$

Set the initial conditions and disturbances as as

$$
\begin{aligned}
x[k] & =\left[\begin{array}{lllllllll}
1 & 2 & 3 & 4 & 5 & 6 & 7 & 8 & 9
\end{array}\right]^{T} \\
\bar{x}[k] & =\left[\begin{array}{lllllllll}
0 & 0 & 0 & 0 & 0 & 0 & 0 & 0 & 0
\end{array}\right]^{T} \\
w[k] & =\left[\begin{array}{llllll}
0 & 0 & 0 & 0 & 0
\end{array}\right]^{T} \\
d_{1}[k] & =1(\text { for } k \geq 0) \\
d_{2}[k] & =1(\text { for } k \geq 0) .
\end{aligned}
$$

The simulation results in Figure 6.8 show that we successfully achieve deadbeat disturbance rejection.

\subsection{Wheel Structure}

We consider two wheel-structure systems in this section. A wheel structure is similar to a ring structure, but it has a center plant agent connected to all other plant agents, which greatly reduces 


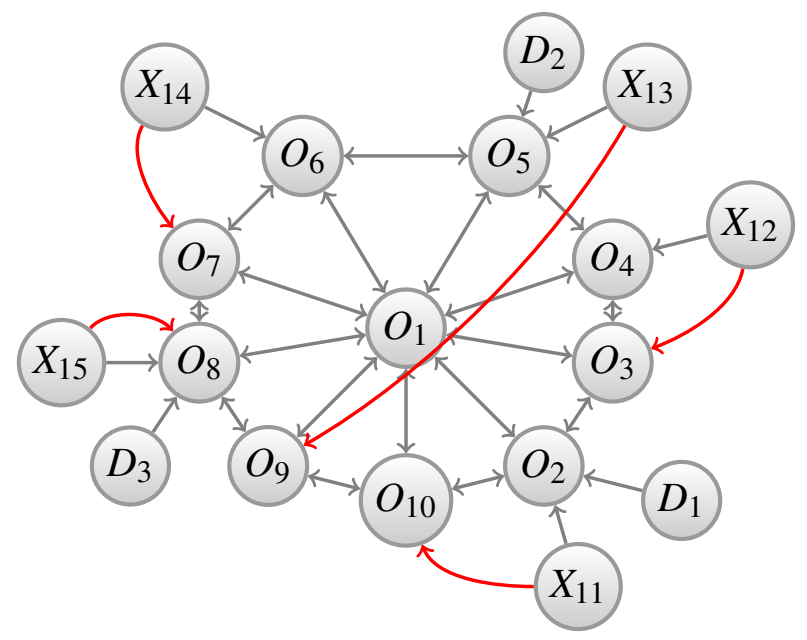

Figure 6.9: The first wheel structure system with ten plant agents and three disturbance agents.

some propagation times. The first example is a wheel structure system with ten plant agents and three disturbance agents, shown in Figure 6.9. The dynamics of this system are as follows:

$$
\begin{aligned}
x_{1}[k+1] & =x_{2}[k]+x_{3}[k]+x_{4}[k]+x_{5}[k]+x_{6}[k]+x_{7}[k]+x_{8}[k]+x_{9}[k]+x_{10}[k] \\
x_{2}[k+1] & =x_{1}[k]+x_{3}[k]+x_{10}[k]+u_{11}[k]+d_{1}[k] \\
x_{3}[k+1] & =x_{1}[k]+x_{2}[k]+x_{4}[k] \\
x_{4}[k+1] & =x_{1}[k]+x_{3}[k]+x_{5}[k]+u_{12}[k] \\
x_{5}[k+1] & =x_{1}[k]+x_{4}[k]+x_{6}[k]+u_{13}[k]+d_{2}[k] \\
x_{6}[k+1] & =x_{1}[k]+x_{5}[k]+x_{7}[k]+u_{14}[k] \\
x_{7}[k+1] & =x_{1}[k]+x_{6}[k]+x_{8}[k] \\
x_{8}[k+1] & =x_{1}[k]+x_{7}[k]+x_{9}[k]+u_{15}[k]+d_{3}[k] \\
x_{9}[k+1] & =x_{1}[k]+x_{8}[k]+x_{10}[k] \\
x_{10}[k+1] & =x_{1}[k]+x_{2}[k]+x_{9}[k] .
\end{aligned}
$$

According to Lemma 1 , three control agents $\left(X_{11}, X_{13}\right.$, and $\left.X_{15}\right)$ need to be placed beside the disturbance agents. To satisfy Theorem 3 and 4 in this case, at least two more control agents $\left(X_{14}\right.$ and $X_{12}$ ) are needed. We assign $O_{9}$ as the target of $X_{13}$ so that the center agent $O_{1}$ lies on the fastest path from $X_{13}$ to $O_{9}$. The reader can verify that both targeting and growing succeed for this setup.

The propagation time for each fastest path from $X_{i}$ to $T_{i}$ (for $11 \leq i \leq 15$ ) is

$$
\begin{aligned}
\Delta\left(X_{11}, O_{10}\right) & =2 \\
\Delta\left(X_{12}, O_{3}\right) & =2
\end{aligned}
$$




$$
\begin{aligned}
& \Delta\left(X_{13}, O_{9}\right)=3 \\
& \Delta\left(X_{14}, O_{7}\right)=2 \\
& \Delta\left(X_{15}, O_{8}\right)=1,
\end{aligned}
$$

and therefore we have

$$
\begin{aligned}
x_{8}[k+1]= & x_{1}[k]+x_{7}[k]+x_{9}[k]+u_{15}[k] \\
= & v_{15}[k] \\
x_{10}[k+2]= & x_{1}[k+1]+x_{9}[k+1]+x_{2}[k+1] \\
= & 2 x_{1}[k]+x_{2}[k]+2 x_{3}[k]+x_{4}[k]+x_{5}[k]+x_{6}[k] \\
& +x_{7}[k]+2 x_{8}[k]+x_{9}[k]+3 x_{10}[k]+u_{11}[k] \\
= & v_{11}[k] \\
x_{3}[k+2]= & x_{1}[k+1]+x_{2}[k+1]+x_{4}[k+1] \\
= & 2 x_{1}[k]+x_{2}[k]+3 x_{3}[k]+x_{4}[k]+2 x_{5}[k]+x_{6}[k] \\
& +x_{7}[k]+x_{8}[k]+x_{9}[k]+2 x_{10}[k]+u_{11}[k]+u_{12}[k] \\
= & v_{12}[k] \\
x_{7}[k+2]= & x_{1}[k+1]+x_{6}[k+1]+x_{8}[k+1] \\
= & 2 x_{1}[k]+x_{2}[k]+x_{3}[k]+x_{4}[k]+2 x_{5}[k]+x_{6}[k] \\
& +3 x_{7}[k]+x_{8}[k]+2 x_{9}[k]+x_{10}[k]+u_{15}[k]+u_{14}[k] \\
= & v_{14}[k] \\
x_{9}[k+3]= & x_{1}[k+2]+x_{8}[k+2]+x_{10}[k+2] \\
= & x_{1}[k+1]+2 x_{2}[k+1]+x_{3}[k+1]+x_{4}[k+1]+x_{5}[k+1]+x_{6}[k+1] \\
& +x_{7}[k+1]+x_{8}[k+1]+2 x_{9}[k+1]+x_{10}[k+1] \\
= & 11 x_{1}[k]+3 x_{2}[k]+4 x_{3}[k]+3 x_{4}[k]+3 x_{6}[k]+3 x_{7}[k]+4 x_{8}[k]+3 x_{9}[k] \\
& +5 x_{10}[k]+2 u_{11}[k]+u_{12}[k]+u_{13}[k]+u_{14}[k]+u_{15}[k] \\
= & v_{13}[k] .
\end{aligned}
$$

Use these results to derive the control laws $u[k]$ :

$$
\begin{aligned}
u_{15}[k]= & v_{15}[k]-x_{1}[k]-x_{7}[k]-x_{9}[k] \\
u_{11}[k]= & v_{11}[k]-2 x_{1}[k]-x_{2}[k]-2 x_{3}[k]-x_{4}[k]-x_{5}[k] \\
& -x_{6}[k]-x_{7}[k]-2 x_{8}[k]-x_{9}[k]-3 x_{10}[k] \\
u_{12}[k]= & v_{12}[k]-2 x_{1}[k]-x_{2}[k]-3 x_{3}[k]-x_{4}[k]-2 x_{5}[k] \\
& -x_{6}[k]-x_{7}[k]-x_{8}[k]-x_{9}[k]-2 x_{10}[k]-u_{11}[k] \\
u_{14}[k]= & v_{14}[k]-2 x_{1}[k]-x_{2}[k]-x_{3}[k]-x_{4}[k]-2 x_{5}[k]
\end{aligned}
$$




$$
\begin{aligned}
& -x_{6}[k]-3 x_{7}[k]-x_{8}[k]-2 x_{9}[k]-x_{10}[k]-u_{15}[k] \\
u_{13}[k]= & v_{13}[k]-11 x_{1}[k]-3 x_{2}[k]-4 x_{3}[k]-3 x_{4}[k]-3 x_{6}[k]-3 x_{7}[k] \\
& -4 x_{8}[k]-3 x_{9}[k]-5 x_{10}[k]-2 u_{11}[k]-u_{12}[k]-u_{14}[k]-u_{15}[k] .
\end{aligned}
$$

From the propagation time $\Delta\left(X_{i}, T_{i}\right)$ (for $11 \leq i \leq 15$ ), we can find the matrices of $K_{1}$ and $K_{2}$ :

$$
K_{1}=\left[\begin{array}{llllllllll}
1 & 1 & 0 & 0 & 0 & 0 & 0 & 0 & 0 & 0 \\
0 & 0 & 1 & 1 & 0 & 0 & 0 & 0 & 0 & 0 \\
0 & 0 & 0 & 0 & 1 & 1 & 1 & 0 & 0 & 0 \\
0 & 0 & 0 & 0 & 0 & 0 & 0 & 1 & 1 & 0 \\
0 & 0 & 0 & 0 & 0 & 0 & 0 & 0 & 0 & 1
\end{array}\right], K_{2}=\left[\begin{array}{lllll}
1 & 0 & 0 & 0 & 0 \\
0 & 1 & 0 & 0 & 0 \\
0 & 0 & 1 & 0 & 0 \\
0 & 0 & 0 & 1 & 0 \\
0 & 0 & 0 & 0 & 1
\end{array}\right]
$$

Thus the control laws of $v[k]$ are

$$
\begin{aligned}
& v_{11}[k]=-\bar{x}_{1}[k]-\bar{x}_{2}[k]-w_{1}[k] \\
& v_{12}[k]=-\bar{x}_{3}[k]-\bar{x}_{4}[k]-w_{2}[k] \\
& v_{13}[k]=-\bar{x}_{5}[k]-\bar{x}_{6}[k]-\bar{x}_{7}[k]-w_{3}[k] \\
& v_{14}[k]=-\bar{x}_{8}[k]-\bar{x}_{9}[k]-w_{4}[k] \\
& v_{15}[k]=-\bar{x}_{10}[k]-w_{5}[k] .
\end{aligned}
$$

Substitute $v[k]$ into the expressions for $u[k]$ to obtain

$$
\begin{aligned}
u_{15}[k]= & v_{15}[k]-x_{1}[k]-x_{7}[k]-x_{9}[k] \\
= & -\bar{x}_{10}[k]-w_{5}[k]-x_{1}[k]-x_{7}[k]-x_{9}[k] \\
u_{11}[k]= & v_{11}[k]-2 x_{1}[k]-x_{2}[k]-2 x_{3}[k]-x_{4}[k]-x_{5}[k] \\
& -x_{6}[k]-x_{7}[k]-2 x_{8}[k]-x_{9}[k]-3 x_{10}[k] \\
= & -\bar{x}_{1}[k]-\bar{x}_{2}[k]-w_{1}[k]-2 x_{1}[k]-x_{2}[k]-2 x_{3}[k] \\
& -x_{4}[k]-x_{5}[k]-x_{6}[k]-x_{7}[k]-2 x_{8}[k]-x_{9}[k]-3 x_{10}[k] \\
u_{12}[k]= & v_{12}[k]-2 x_{1}[k]-x_{2}[k]-3 x_{3}[k]-x_{4}[k]-2 x_{5}[k] \\
& -x_{6}[k]-x_{7}[k]-x_{8}[k]-x_{9}[k]-2 x_{10}[k]-u_{11}[k] \\
= & -\bar{x}_{3}[k]-\bar{x}_{4}[k]-w_{2}[k]-2 x_{1}[k]-x_{2}[k]-3 x_{3}[k]-x_{4}[k] \\
& -2 x_{5}[k]-x_{6}[k]-x_{7}[k]-x_{8}[k]-x_{9}[k]-2 x_{10}[k]-u_{11}[k] \\
u_{14}[k]= & v_{14}[k]-2 x_{1}[k]-x_{2}[k]-x_{3}[k]-x_{4}[k]-2 x_{5}[k] \\
& -x_{6}[k]-3 x_{7}[k]-x_{8}[k]-2 x_{9}[k]-x_{10}[k]-u_{15}[k] \\
= & -\bar{x}_{8}[k]-\bar{x}_{9}[k]-w_{4}[k]-2 x_{1}[k]-x_{2}[k]-x_{3}[k]-x_{4}[k] \\
& -2 x_{5}[k]-x_{6}[k]-3 x_{7}[k]-x_{8}[k]-2 x_{9}[k]-x_{10}[k]-u_{15}[k] \\
u_{13}[k]= & v_{13}[k]-11 x_{1}[k]-3 x_{2}[k]-4 x_{3}[k]-3 x_{4}[k]-3 x_{6}[k]-3 x_{7}[k]-4 x_{8}[k]
\end{aligned}
$$




$$
\begin{aligned}
& -3 x_{9}[k]-5 x_{10}[k]-2 u_{11}[k]-u_{12}[k]-u_{14}[k]-u_{15}[k] \\
= & -\bar{x}_{5}[k]-\bar{x}_{6}[k]-\bar{x}_{7}[k]-w_{3}[k]-11 x_{1}[k]-3 x_{2}[k] \\
& -4 x_{3}[k]-3 x_{4}[k]-3 x_{6}[k]-3 x_{7}[k]-4 x_{8}[k]-3 x_{9}[k]-5 x_{10}[k] \\
& -2 u_{11}[k]-u_{12}[k]-u_{14}[k]-u_{15}[k],
\end{aligned}
$$

where

$$
\begin{aligned}
\bar{x}[k]= & {\left[v_{11}[k-2], v_{11}[k-1], v_{12}[k-2], v_{12}[k-1], v_{13}[k-3],\right.} \\
& \left.v_{13}[k-2], v_{13}[k-1], v_{14}[k-2], v_{14}[k-1], v_{15}[k-1]\right]^{T} .
\end{aligned}
$$

Set the initial conditions and disturbance signals to

$$
\begin{aligned}
x[k] & =\left[\begin{array}{llllllllll}
1 & 2 & 3 & 4 & 5 & 6 & 7 & 8 & 9 & 10
\end{array}\right]^{T} \\
\bar{x}[k] & =\left[\begin{array}{llllllllll}
0 & 0 & 0 & 0 & 0 & 0 & 0 & 0 & 0 & 0
\end{array}\right]^{T} \\
w[k] & =\left[\begin{array}{lllll}
0 & 0 & 0 & 0 & 0
\end{array}\right]^{T} \\
d_{1}[k] & =1(\text { for } k \geq 0) \\
d_{2}[k] & =1(\text { for } k \geq 0) .
\end{aligned}
$$

The simulation results in Figure 6.10 show that deadbeat disturbance rejection is successfully achieved.

The second wheel structure system has five plant agents and two disturbance agents. However, there is a disturbance agent connected to the center plant agent in this example. The dynamical equations of this system are

$$
\begin{aligned}
& x_{1}[k+1]=2 x_{2}[k]+x_{4}[k]+x_{5}[k] \\
& x_{2}[k+1]=x_{1}[k]+x_{3}[k]+x_{5}[k] \\
& x_{3}[k+1]=x_{2}[k]-x_{4}[k]+x_{5}[k]+u_{7}[k] \\
& x_{4}[k+1]=x_{1}[k]+3 x_{3}[k]+x_{5}[k]+u_{8}[k]+d_{1}[k] \\
& x_{5}[k+1]=x_{1}[k]+x_{2}[k]+x_{3}[k]+x_{4}[k]+u_{6}[k]+d_{2}[k] .
\end{aligned}
$$

As required by Lemma 1, we place a control agent $X_{6}$ beside $D_{2}$, connected to $O_{5}$. Similarly, we connect another control agent $X_{8}$ to $O_{4}$ because of $D_{1}$. To satisfy Theorem 3 and 4, one more control agent $X_{7}$ is connected to $O_{3}$. For growing to succeed, we assign $O_{1}$ as $T_{8}, O_{2}$ as $T_{7}$ and $O_{5}$ as $T_{6}$. With this arrangement, both targeting and growing succeed.

The propagation times from $X_{i}$ to $T_{i}$ (for $6 \leq i \leq 8$ ) are

$$
\begin{aligned}
& \Delta\left(X_{6}, O_{5}\right)=1 \\
& \Delta\left(X_{7}, O_{2}\right)=2 \\
& \Delta\left(X_{8}, O_{1}\right)=2,
\end{aligned}
$$



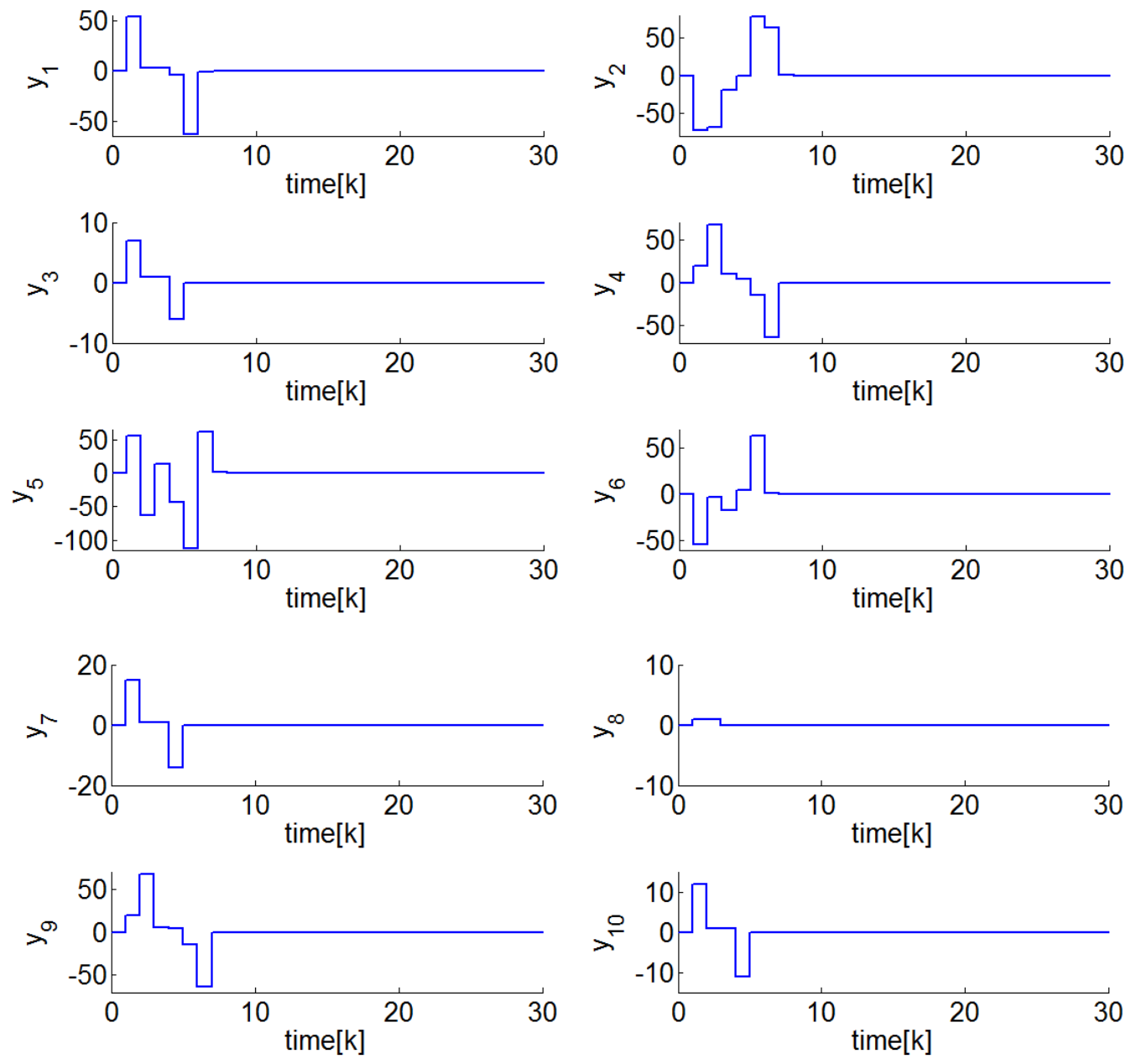

Figure 6.10: Simulation results for the first wheel structure system example. 


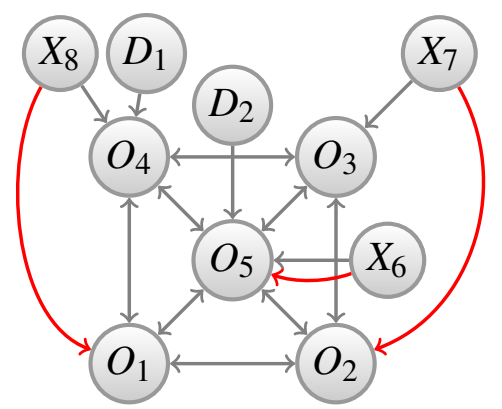

Figure 6.11: The second wheel structure system with five plant agents and two disturbance agents.

and we have

$$
\begin{aligned}
x_{5}[k+1] & =x_{1}[k]+x_{2}[k]+x_{3}[k]+x_{4}[k]+u_{6}[k] \\
& =v_{6}[k] \\
x_{2}[k+2] & =x_{1}[k+1]+x_{3}[k+1]+x_{5}[k+1] \\
& =x_{1}[k]+4 x_{2}[k]+x_{3}[k]+x_{4}[k]+2 x_{5}[k]+u_{6}[k]+u_{7}[k] \\
& =v_{7}[k] \\
x_{1}[k+2] & =2 x_{2}[k+1]+x_{4}[k+1]+x_{5}[k+1] \\
& =4 x_{1}[k]+x_{2}[k]+6 x_{3}[k]+x_{4}[k]+3 x_{5}[k]+u_{6}[k]+u_{8}[k] \\
& =v_{8}[k] .
\end{aligned}
$$

Hence, the control laws $u[k]$ are

$$
\begin{aligned}
u_{6}[k] & =v_{6}[k]-x_{1}[k]-x_{2}[k]-x_{3}[k]-x_{4}[k] \\
u_{7}[k] & =v_{7}[k]-x_{1}[k]-4 x_{2}[k]-x_{3}[k]-x_{4}[k]-2 x_{5}[k]-u_{6}[k] \\
u_{8}[k] & =v_{8}[k]-4 x_{1}[k]-x_{2}[k]-6 x_{3}[k]-x_{4}[k]-3 x_{5}[k]-u_{6}[k] .
\end{aligned}
$$

The controller gain matrices $K_{1}$ and $K_{2}$ are

$$
K_{1}=\left[\begin{array}{lllll}
1 & 0 & 0 & 0 & 0 \\
0 & 1 & 1 & 0 & 0 \\
0 & 0 & 0 & 1 & 1
\end{array}\right], K_{2}=\left[\begin{array}{lll}
1 & 0 & 0 \\
0 & 1 & 0 \\
0 & 0 & 1
\end{array}\right]
$$

while the control laws for $v[k]$ are

$$
\begin{aligned}
& v_{6}[k]=-\bar{x}_{1}[k]-w_{1}[k] \\
& v_{7}[k]=-\bar{x}_{2}[k]-\bar{x}_{3}[k]-w_{2}[k] \\
& v_{8}[k]=-\bar{x}_{4}[k]-\bar{x}_{5}[k]-w_{3}[k] .
\end{aligned}
$$


Substitute $v[k]$ into the formula for $u[k]$ in 6.93-6.95

$$
\begin{aligned}
u_{6}[k]= & v_{6}[k]-x_{1}[k]-x_{2}[k]-x_{3}[k]-x_{4}[k] \\
= & -\bar{x}_{1}[k]-w_{1}[k]-x_{1}[k]-x_{2}[k]-x_{3}[k]-x_{4}[k] \\
u_{7}[k]= & v_{7}[k]-x_{1}[k]-4 x_{2}[k]-x_{3}[k]-x_{4}[k]-2 x_{5}[k]-u_{6}[k] \\
= & -\bar{x}_{2}[k]-\bar{x}_{3}[k]-w_{2}[k]-x_{1}[k]-4 x_{2}[k] \\
& -x_{3}[k]-x_{4}[k]-2 x_{5}[k]-u_{6}[k] \\
u_{8}[k]= & v_{8}[k]-4 x_{1}[k]-x_{2}[k]-6 x_{3}[k]-x_{4}[k]-3 x_{5}[k]-u_{6}[k] \\
= & -\bar{x}_{4}[k]-\bar{x}_{5}[k]-w_{3}[k]-4 x_{1}[k]-x_{2}[k] \\
& -6 x_{3}[k]-x_{4}[k]-3 x_{5}[k]-u_{6}[k],
\end{aligned}
$$

where

$$
\bar{x}[k]=\left[v_{6}[k-1], v_{7}[k-2], v_{7}[k-1], v_{8}[k-2], v_{8}[k-1]\right]^{T} .
$$

Finally, set the initial conditions as

$$
\begin{aligned}
x[k] & =\left[\begin{array}{lllll}
1 & 2 & 3 & 4 & 5
\end{array}\right]^{T} \\
\bar{x}[k] & =\left[\begin{array}{lllll}
0 & 0 & 0 & 0 & 0
\end{array}\right]^{T} \\
w[k] & =\left[\begin{array}{lll}
0 & 0 & 0
\end{array}\right]^{T} \\
d_{1}[k] & =1(\text { for } k \geq 0) \\
d_{2}[k] & =1(\text { for } k \geq 0) .
\end{aligned}
$$

Simulation results in Figure 6.12 show, once again, that we successfully achieve deadbeat disturbance rejection.

\subsection{Complete Graph Structure}

Consider the system in Figure 6.13, a plant that has a complete graph structure where each plant agent is connected to all the other plant agents. This system has six plant agents and three disturbance agents. The dynamics are

$$
\begin{aligned}
x_{1}[k+1] & =x_{1}[k]+x_{2}[k]+x_{3}[k]+x_{4}[k]+x_{5}[k]+x_{6}[k] \\
x_{2}[k+1] & =x_{1}[k]+x_{3}[k]+x_{4}[k]+x_{5}[k]+x_{6}[k]+u_{7}[k]+d_{1}[k] \\
x_{3}[k+1] & =x_{1}[k]+x_{2}[k]+x_{4}[k]+x_{5}[k]+x_{6}[k]+u_{8}[k] \\
x_{4}[k+1] & =x_{1}[k]+x_{2}[k]+x_{3}[k]+x_{5}[k]+x_{6}[k]+u_{9}[k]+d_{2}[k] \\
x_{5}[k+1] & =x_{1}[k]+x_{2}[k]+x_{3}[k]+x_{4}[k]+x_{6}[k]+u_{10}[k]+d_{3}[k] \\
x_{6}[k+1] & =x_{1}[k]+x_{2}[k]+x_{3}[k]+x_{4}[k]+x_{5}[k]+u_{11}[k] .
\end{aligned}
$$



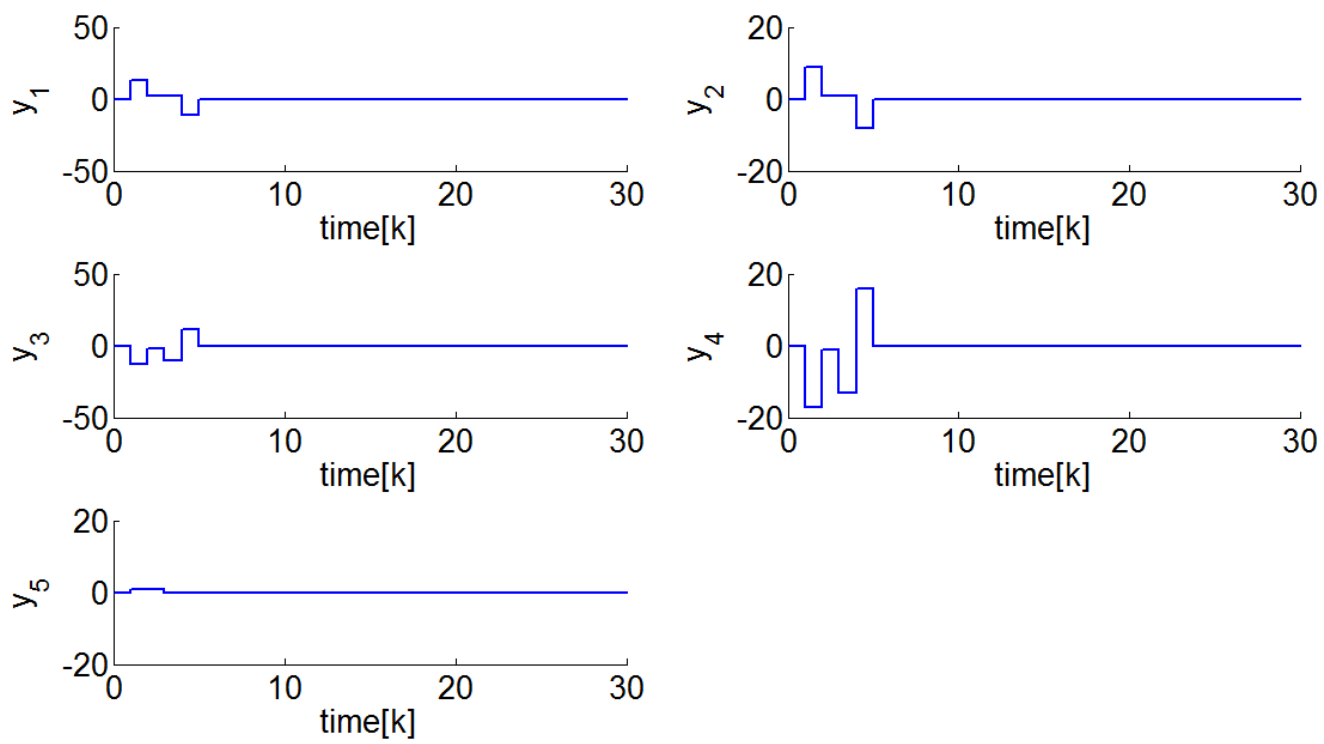

Figure 6.12: Simulation result for the second wheel structure system example.

We assign five control agents and their targets to satisfy Lemma 1, Theorem 3, and Theorem 4. Targeting and growing both succeed.

We determine the propagation time from the control agents to their targets to be

$$
\begin{aligned}
\Delta\left(X_{7}, O_{1}\right) & =2 \\
\Delta\left(X_{8}, O_{3}\right) & =1 \\
\Delta\left(X_{9}, O_{4}\right) & =1 \\
\Delta\left(X_{10}, O_{5}\right) & =1 \\
\Delta\left(X_{11}, O_{6}\right) & =1,
\end{aligned}
$$

and therefore

$$
\begin{aligned}
x_{3}[k+1] & =x_{1}[k]+x_{2}[k]+x_{4}[k]+x_{5}[k]+x_{6}[k]+u_{8}[k] \\
& =v_{8}[k] \\
x_{4}[k+1] & =x_{1}[k]+x_{2}[k]+x_{3}[k]+x_{5}[k]+x_{6}[k]+u_{9}[k] \\
& =v_{9}[k] \\
x_{5}[k+1] & =x_{1}[k]+x_{2}[k]+x_{3}[k]+x_{4}[k]+x_{6}[k]+u_{10}[k] \\
& =v_{10}[k] \\
x_{6}[k+1] & =x_{1}[k]+x_{2}[k]+x_{3}[k]+x_{4}[k]+x_{5}[k]+u_{11}[k]
\end{aligned}
$$




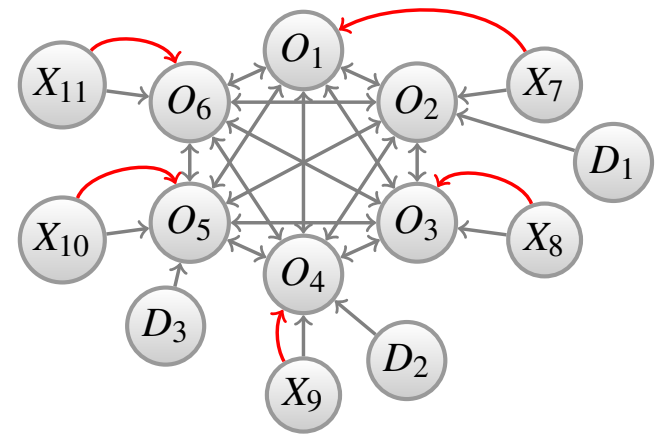

Figure 6.13: The complete graph structure system with six plant agents and three disturbance agents.

$$
\begin{aligned}
= & v_{11}[k] \\
x_{1}[k+2]= & x_{1}[k+1]+x_{2}[k+1]+x_{3}[k+1]+x_{4}[k+1]+x_{5}[k+1]+x_{6}[k+1] \\
= & 6 x_{1}[k]+5 x_{2}[k]+5 x_{3}[k]+5 x_{4}[k]+5 x_{5}[k]+5 x_{6}[k] \\
& +u_{7}[k]+u_{8}[k]+u_{9}[k]+u_{10}[k] \\
= & v_{7}[k] .
\end{aligned}
$$

Next, derive the control laws $u[k]$

$$
\begin{aligned}
u_{8}[k]= & v_{8}[k]-x_{1}[k]-x_{2}[k]-x_{4}[k]-x_{5}[k]-x_{6}[k] \\
u_{9}[k]= & v_{9}[k]-x_{1}[k]-x_{2}[k]-x_{3}[k]-x_{5}[k]-x_{6}[k] \\
u_{10}[k]= & v_{10}[k]-x_{1}[k]-x_{2}[k]-x_{3}[k]-x_{4}[k]-x_{6}[k] \\
u_{11}[k]= & v_{11}[k]-x_{1}[k]-x_{2}[k]-x_{3}[k]-x_{4}[k]-x_{5}[k] \\
u_{7}[k]= & v_{7}[k]-6 x_{1}[k]-5 x_{2}[k]-5 x_{3}[k]-5 x_{4}[k]-5 x_{5}[k] \\
& -5 x_{6}[k]-u_{8}[k]-u_{9}[k]-u_{10}[k]
\end{aligned}
$$

and the matrices of $K_{1}$ and $K_{2}$ :

$$
K_{1}=\left[\begin{array}{llllll}
1 & 1 & 0 & 0 & 0 & 0 \\
0 & 0 & 1 & 0 & 0 & 0 \\
0 & 0 & 0 & 1 & 0 & 0 \\
0 & 0 & 0 & 0 & 1 & 0 \\
0 & 0 & 0 & 0 & 0 & 1
\end{array}\right], K_{2}=\left[\begin{array}{lllll}
1 & 0 & 0 & 0 & 0 \\
0 & 1 & 0 & 0 & 0 \\
0 & 0 & 1 & 0 & 0 \\
0 & 0 & 0 & 1 & 0 \\
0 & 0 & 0 & 0 & 1
\end{array}\right] .
$$

The control laws $v[k]$ are

$$
\begin{aligned}
& v_{7}[k]=-\bar{x}_{1}[k]-\bar{x}_{2}[k]-w_{1}[k] \\
& v_{8}[k]=-\bar{x}_{3}[k]-w_{2}[k]
\end{aligned}
$$




$$
\begin{aligned}
v_{9}[k] & =-\bar{x}_{4}[k]-w_{3}[k] \\
v_{10}[k] & =-\bar{x}_{5}[k]-w_{4}[k] \\
v_{11}[k] & =-\bar{x}_{6}[k]-w_{5}[k] .
\end{aligned}
$$

Substitute $v[k]$ into the expressions for $u[k]$ to obtain

$$
\begin{aligned}
u_{8}[k]= & v_{8}[k]-x_{1}[k]-x_{2}[k]-x_{4}[k]-x_{5}[k]-x_{6}[k] \\
= & -\bar{x}_{3}[k]-w_{2}[k]-x_{1}[k]-x_{2}[k]-x_{4}[k]-x_{5}[k]-x_{6}[k] \\
u_{9}[k]= & v_{9}[k]-x_{1}[k]-x_{2}[k]-x_{3}[k]-x_{5}[k]-x_{6}[k] \\
= & -\bar{x}_{4}[k]-w_{3}[k]-x_{1}[k]-x_{2}[k]-x_{3}[k]-x_{5}[k]-x_{6}[k] \\
u_{10}[k]= & v_{10}[k]-x_{1}[k]-x_{2}[k]-x_{3}[k]-x_{4}[k]-x_{6}[k] \\
= & -\bar{x}_{5}[k]-w_{4}[k]-x_{1}[k]-x_{2}[k]-x_{3}[k]-x_{4}[k]-x_{6}[k] \\
u_{11}[k]= & v_{11}[k]-x_{1}[k]-x_{2}[k]-x_{3}[k]-x_{4}[k]-x_{5}[k] \\
= & -\bar{x}_{6}[k]-w_{5}[k]-x_{1}[k]-x_{2}[k]-x_{3}[k]-x_{4}[k]-x_{5}[k] \\
u_{7}[k]= & v_{7}[k]-6 x_{1}[k]-5 x_{2}[k]-5 x_{3}[k]-5 x_{4}[k]-5 x_{5}[k] \\
& -5 x_{6}[k]-u_{8}[k]-u_{9}[k]-u_{10}[k] \\
= & -\bar{x}_{1}[k]-\bar{x}_{2}[k]-w_{1}[k]-6 x_{1}[k]-5 x_{2}[k]-5 x_{3}[k] \\
& -5 x_{4}[k]-5 x_{5}[k]-5 x_{6}[k]-u_{8}[k]-u_{9}[k]-u_{10}[k],
\end{aligned}
$$

where

$$
\bar{x}[k]=\left[v_{7}[k-2], v_{7}[k-1], v_{8}[k-1], v_{9}[k-1], v_{10}[k-1], v_{11}[k-1]\right]^{T} .
$$

For simulation purposes, set the initial conditions and disturbances as

$$
\begin{aligned}
x[k] & =\left[\begin{array}{llllll}
1 & 2 & 3 & 4 & 5 & 6
\end{array}\right]^{T} \\
\bar{x}[k] & =\left[\begin{array}{llllll}
0 & 0 & 0 & 0 & 0 & 0
\end{array}\right]^{T} \\
w[k] & =\left[\begin{array}{lllll}
0 & 0 & 0 & 0 & 0
\end{array}\right]^{T} \\
d_{1}[k] & =1(\text { for } k \geq 0) \\
d_{2}[k] & =1(\text { for } k \geq 0) .
\end{aligned}
$$

From the simulation results in Figure 6.14, we see that the algorithm works in the complete graph structure system.

\subsection{Null Graph Structure}

Consider the other extreme case, namely a null graph structure where the plant agents have no connections to one another. An example of a null graph structure system with six plant agents 

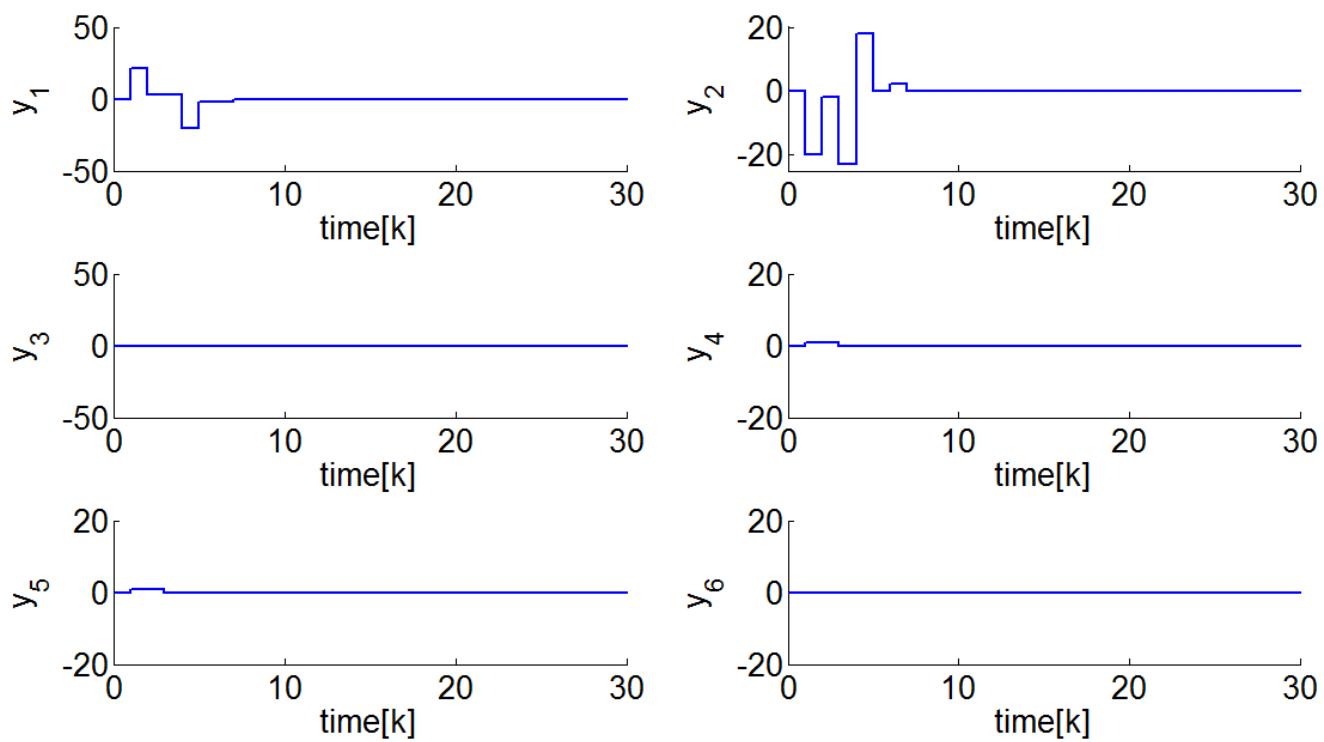

Figure 6.14: Simulation result for the complete graph structure system example.

and six disturbance agents is shown in Figure 6.15. The dynamics are taken to be

$$
\begin{aligned}
x_{1}[k+1] & =2 x_{1}[k]+u_{7}[k]+d_{1}[k] \\
x_{2}[k+1] & =x_{2}[k]+u_{8}[k]+d_{2}[k] \\
x_{3}[k+1] & =x_{3}[k]+u_{9}[k]+d_{3}[k] \\
x_{4}[k+1] & =x_{4}[k]+u_{10}[k]+d_{4}[k] \\
x_{5}[k+1] & =x_{5}[k]+u_{11}[k]+d_{5}[k] \\
x_{6}[k+1] & =x_{6}[k]+u_{12}[k]+d_{6}[k] .
\end{aligned}
$$

As all the propagation times from control agents to respective targets are one, we can directly derive the control laws of $u[k]$ based on $6.112-6.117$ :

$$
\begin{aligned}
u_{7}[k] & =v_{7}[k]-2 x_{1}[k] \\
u_{8}[k] & =v_{8}[k]-x_{2}[k] \\
u_{9}[k] & =v_{9}[k]-x_{3}[k] \\
u_{10}[k] & =v_{10}[k]-x_{4}[k] \\
u_{11}[k] & =v_{11}[k]-x_{5}[k] \\
u_{12}[k] & =v_{12}[k]-x_{6}[k] .
\end{aligned}
$$




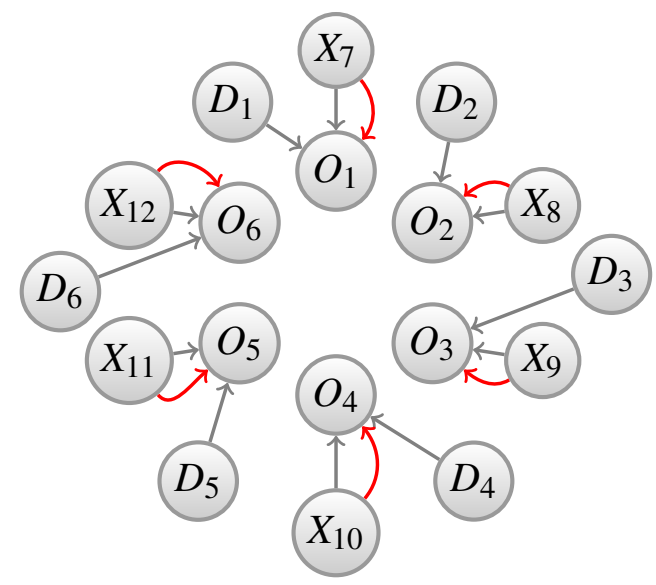

Figure 6.15: The null graph structure system with six plant agents and six disturbance agents.

Find the matrices of $K_{1}$ and $K_{2}$

$$
K_{1}=\left[\begin{array}{llllll}
1 & 0 & 0 & 0 & 0 & 0 \\
0 & 1 & 0 & 0 & 0 & 0 \\
0 & 0 & 1 & 0 & 0 & 0 \\
0 & 0 & 0 & 1 & 0 & 0 \\
0 & 0 & 0 & 0 & 1 & 0 \\
0 & 0 & 0 & 0 & 0 & 1
\end{array}\right], K_{2}=\left[\begin{array}{llllll}
1 & 0 & 0 & 0 & 0 & 0 \\
0 & 1 & 0 & 0 & 0 & 0 \\
0 & 0 & 1 & 0 & 0 & 0 \\
0 & 0 & 0 & 1 & 0 & 0 \\
0 & 0 & 0 & 0 & 1 & 0 \\
0 & 0 & 0 & 0 & 0 & 1
\end{array}\right]
$$

and the control laws of $v[k]$ :

$$
\begin{aligned}
v_{7}[k] & =-\bar{x}_{1}[k]-w_{1}[k] \\
v_{8}[k] & =-\bar{x}_{2}[k]-w_{2}[k] \\
v_{9}[k] & =-\bar{x}_{3}[k]-w_{3}[k] \\
v_{10}[k] & =-\bar{x}_{4}[k]-w_{4}[k] \\
v_{11}[k] & =-\bar{x}_{5}[k]-w_{5}[k] \\
v_{12}[k] & =-\bar{x}_{6}[k]-w_{6}[k] .
\end{aligned}
$$

Substitute $v[k]$ into the expressions for $u[k]$

$$
\begin{aligned}
u_{7}[k] & =v_{7}[k]-2 x_{1}[k] \\
& =-\bar{x}_{1}[k]-w_{1}[k]-2 x_{1}[k] \\
u_{8}[k] & =v_{8}[k]-x_{2}[k] \\
& =-\bar{x}_{2}[k]-w_{2}[k]-x_{2}[k] \\
u_{9}[k] & =v_{9}[k]-x_{3}[k]
\end{aligned}
$$




$$
\begin{aligned}
& =-\bar{x}_{3}[k]-w_{3}[k]-x_{3}[k] \\
u_{10}[k] & =v_{10}[k]-x_{4}[k] \\
& =-\bar{x}_{4}[k]-w_{4}[k]-x_{4}[k] \\
u_{11}[k] & =v_{11}[k]-x_{5}[k] \\
& =-\bar{x}_{5}[k]-w_{5}[k]-x_{5}[k] \\
u_{12}[k] & =v_{12}[k]-x_{6}[k] \\
& =-\bar{x}_{6}[k]-w_{6}[k]-x_{6}[k],
\end{aligned}
$$

where

$$
\bar{x}[k]=\left[v_{7}[k-1], v_{8}[k-1], v_{9}[k-1], v_{10}[k-1], v_{11}[k-1], v_{12}[k-1]\right]^{T} .
$$

Set the initial conditions and disturbance signals as

$$
\begin{aligned}
x[k] & =\left[\begin{array}{llllll}
1 & 2 & 3 & 4 & 5 & 6
\end{array}\right]^{T} \\
\bar{x}[k] & =\left[\begin{array}{llllll}
0 & 0 & 0 & 0 & 0 & 0
\end{array}\right]^{T} \\
w[k] & =\left[\begin{array}{lllll}
0 & 0 & 0 & 0 & 0
\end{array}\right]^{T} \\
d_{1}[k] & =1(\text { for } k \geq 0) \\
d_{2}[k] & =1(\text { for } k \geq 0) .
\end{aligned}
$$

From the simulation results in Figure 6.16, we see that deadbeat disturbance rejection is obtained. 

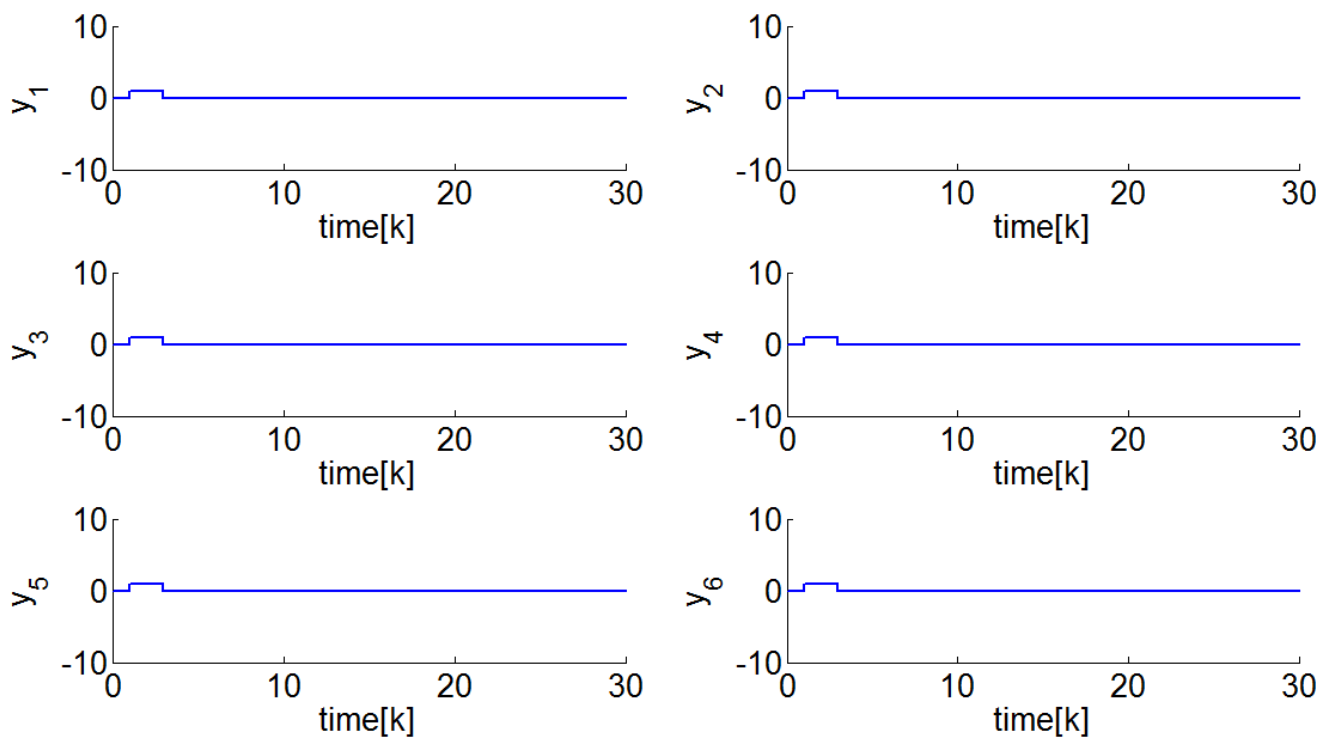

Figure 6.16: Simulation result for the null graph structure system example. 


\section{Chapter 7}

\section{Deadbeat Disturbance Rejection for a Class of Nonlinear Systems}

In the last two chapters, the deadbeat disturbance rejection problem for linear multi-agent systems was discussed. Several examples in Chapter 6 show that the proposed control approach works well in the linear case, which leaves us the question as to whether or not this approach works for nonlinear systems. In this chapter, we focus on a certain class of nonlinear multi-agent system, where we propose a conjecture that the proposed control approach still works.

\subsection{Three Constraints}

In this section, we introduce three constraints on the nonlinear dynamics given in Chapter 1 (in addition to the existing Assumptions $A_{1}-A_{8}$ ) such that the control algorithm from Chapters 5-6 still manages to achieve deadbeat disturbance rejection. These results are preliminary, and the main result is presented later as a conjecture.

The three constraints we require the nonlinear dynamics to satisfy are summarized here, then discussed below:

Constraint $C_{1}$ : The dynamics of the system have the form

$$
\begin{aligned}
x_{i}[k+1] & =f_{i}(x[k], y[k])+B_{i}^{u} u[k]+B_{i}^{d} d_{i}[k] \\
y_{i}[k] & =C_{i} x_{i}[k]
\end{aligned}
$$

where $f_{i}(x[k], y[k])$ is a nonlinear combination of the plant agent states $x[k]=\left[x_{1}^{T}, \cdots, x_{n}^{T}\right]^{T}$ and output $y[k]=\left[y_{1}, \cdots, y_{n}\right]^{T}$, and where $B_{i}^{u}$ and $B_{i}^{d}$ are scalars. (If there is no control agent or 
disturbance connected to plant agent $O_{i}$, for $1 \leq i \leq n$, then $B_{i}^{u}$ or $B_{i}^{d}$ is set to zero.)

Constraint $C_{2}$ : The output of the targets has the form

$$
y_{i}^{\text {tar }}\left[k+\Delta_{i}\right]=g_{i}\left(x[k], y[k], u_{j \neq i}[k], d_{j \neq i}[k]\right)+P_{i}^{u} u_{i}[k]+P_{i}^{d} d_{i}[k],
$$

where $g_{i}\left(x[k], y[k], u_{j \neq i}[k], d_{j \neq i}[k]\right)$ is a nonlinear expression and where $P_{i}^{u}$ and $P_{i}^{d}$ are scalars.

We can re-package 7.3 in various ways, as follows:

$$
\begin{aligned}
y_{i}^{\text {tar }}\left[k+\Delta_{i}\right] & =g_{i}\left(x[k], y[k], u_{j \neq i}[k], d_{j \neq i}[k]\right)+P_{i}^{u} u_{i}[k]+P_{i}^{d} d_{i}[k] \\
& \triangleq g_{i}^{\prime}\left(x[k], y[k], u_{j \neq i}[k]\right)+P_{i}^{u} u_{i}[k]+\tilde{d}[k] \\
& \triangleq v_{i}[k]+\tilde{d}[k] \\
& \triangleq \tilde{v}_{i}[k] .
\end{aligned}
$$

The term $\tilde{d}[k]$ in 7.4$)$ is the sum of $P_{i}^{d} d_{i}[k]$ and all the terms in $g_{i}\left(x[k], y[k], u_{j \neq i}[k], d_{j \neq i}[k]\right)$ that include disturbances; if all disturbance signals are zero, then $\tilde{d}$ is zero. Note that the term $\tilde{d}[k]$ may be a function of states of plant agents and control signals, and the term $g_{i}^{\prime}\left(x[k], y[k], u_{j \neq i}[k]\right)$ might be zero. We define $v_{i}[k]$ in $(7.5)$ to be $g_{i}^{\prime}\left(x[k], y[k], u_{j \neq i}[k]\right)+P_{i}^{u} u_{i}[k]$, and we define $\tilde{v}_{i}[k]$ in (7.6) to be $v_{i}[k]+\tilde{d}[k]$.

We now follow the same process used in Chapter 5 for the linear case. From (7.4) and (7.5), we can determine the control law $u_{i}[k]$ as

$$
u_{i}[k]=\left(v_{i}[k]-g_{i}^{\prime}\left(x[k], y[k], u_{j \neq i}[k]\right)\right) / P_{i}^{u} .
$$

By substituting the control law $u[k]$ back into (7.4), we find an expression for $y_{i}^{\text {tar }}\left[k+\Delta_{i}\right]$ that does not contain any $u[k]$ terms. In fact, we want this expression to also not be affected by any plant-agent state. Thus, we introduce the last constraint:

Constraint $C_{3}$ : The final expression of the target outputs, in terms of the $v[k]$ signals, has the form

$$
y_{i}^{\text {tar }}\left[k+\Delta_{i}\right]=h_{i}\left(v_{j \neq i}[k], d_{j \neq i}[k]\right)+v_{i}[k]+\beta_{i}^{d} d_{i}[k],
$$

where $h_{i}\left(v_{j \neq i}[k], d_{j \neq i}[k]\right)$ is a nonlinear expression independent of $x[k]$ and where $\beta_{i}^{d}$ is a scalar. Note, in particular, that no plant state terms enter into (7.8).

Constraints $C_{1}-C_{3}$ arose from considering various examples. Take, for instance, the queue plant structure in Figure 7.1. Set $y_{i}[k]=x_{i}[k]$, for $1 \leq i \leq 4$. The propagation time from one agent to any neighbour is one, which implies that

$$
\begin{aligned}
& \Delta\left(X_{5}, O_{4}\right)=2 \\
& \Delta\left(X_{6}, O_{2}\right)=2 .
\end{aligned}
$$




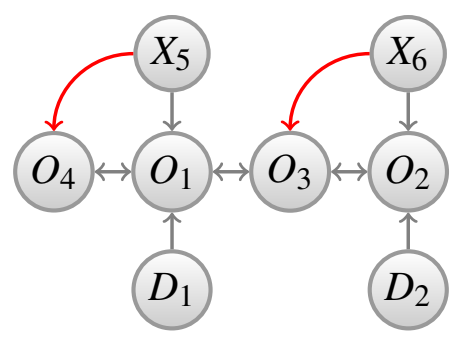

Figure 7.1: A queue structure system with four plant agent and two disturbances.

Let's suppose the queue has the following nonlinear dynamics which does not have the form (7.1):

$$
\begin{aligned}
x_{1}[k+1] & =\left(x_{1}[k]+x_{4}[k]+2 u_{5}[k]+x_{3}^{2}[k]\right) d_{1}[k] \\
x_{2}[k+1] & =\left(-x_{3}[k]+2 u_{6}[k]\right) d_{2}[k] \\
x_{3}[k+1] & =x_{1}^{2}[k]+4 x_{2}[k] \\
x_{4}[k+1] & =3 x_{1}[k] .
\end{aligned}
$$

Disregarding the disturbances, we have

$$
\begin{aligned}
x_{4}[k+2] & =3 x_{1}[k+1] \\
& =3\left(x_{1}[k]+x_{4}[k]+2 u_{5}[k]+x_{3}^{2}[k]\right) \\
& =v_{5}[k] \\
x_{3}[k+2] & =x_{1}^{2}[k+1]+4 x_{2}[k+1] \\
& =\left(x_{1}[k]+x_{4}[k]+2 u_{5}[k]+x_{3}^{2}[k]\right)^{2}+4\left(-x_{3}[k]+2 u_{6}[k]\right) \\
& =v_{6}[k],
\end{aligned}
$$

which leads to

$$
\begin{aligned}
& u_{5}[k]=\left(v_{5}[k]-3 x_{1}[k]-3 x_{4}[k]-3 x_{3}^{2}[k]\right) / 6 \\
& u_{6}[k]=\left(v_{6}[k]-\left(x_{1}[k]+x_{4}[k]+2 u_{5}[k]+x_{3}^{2}[k]\right)^{2}+4 x_{3}[k]\right) / 8 .
\end{aligned}
$$

Substitute the control laws back to the expressions for the outputs of the targets:

$$
\begin{aligned}
y_{4}^{\text {tar }}[k+2] & =3 x_{1}[k+1] \\
& =3\left(x_{1}[k]+x_{4}[k]+2 u_{5}[k]+x_{3}^{2}[k]\right) d_{1}[k] \\
& =v_{5}[k] d_{1}[k] \\
y_{3}^{\text {tar }}[k+2] & =x_{1}^{2}[k+1]+4 x_{2}[k+1] \\
& =\left(x_{1}[k]+x_{4}[k]+2 u_{5}[k]+x_{3}^{2}[k]\right)^{2} d_{1}^{2}[k]+4\left(-x_{3}[k]+2 u_{6}[k]\right) d_{2}[k] \\
& =\left(v_{5}^{2}[k] d_{1}^{2}[k]\right) / 9+\left(v_{6}[k]-v_{5}^{2}[k] / 9\right) d_{2}^{2}[k] .
\end{aligned}
$$



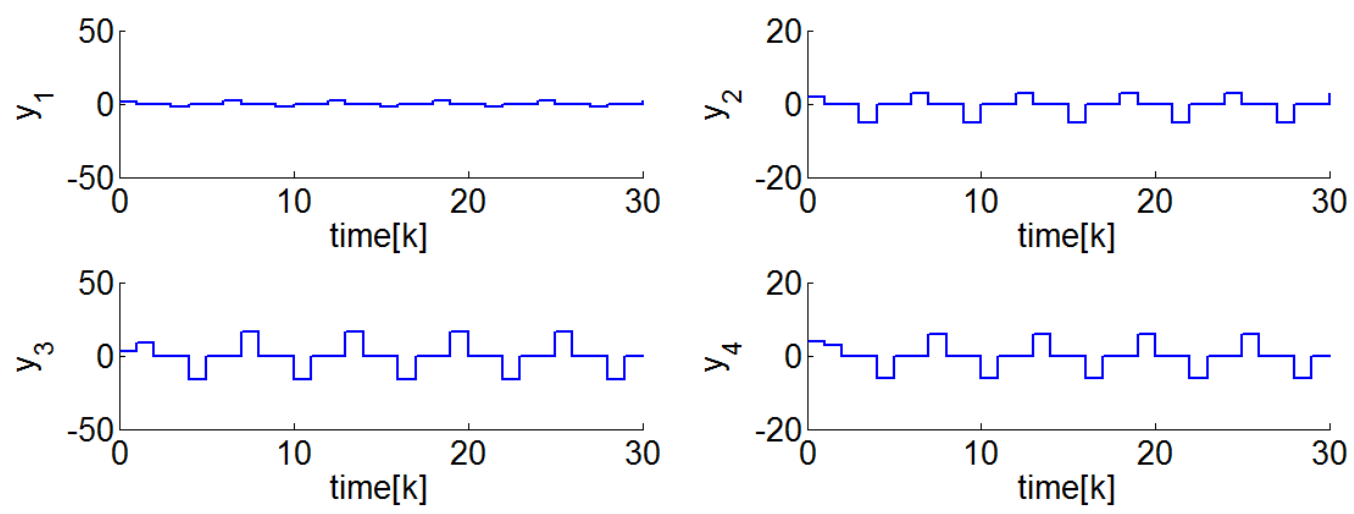

Figure 7.2: Simulation result for the queue plant with dynamics in (7.11)-7.14).

Although the target output (7.17) and (7.18) have the form of (7.8), the dynamics (7.11) and (7.12) do not have the form of (7.1) due to how the disturbances enter the equations. In this example, if both the disturbance signals are equal to one, deadbeat disturbance rejection is (obviously) achieved. But if we set the disturbances to $d_{1}[k]=d_{2}[k]=2$, then the simulation results in Figure 7.2 show that the algorithm does not reject the disturbance. (Moreover, if the disturbances are zero, Assumption $A_{6}$ is violated, but that is not relevant for the point being made here.) Our conclusion is that, to avoid problems of this nature, the safest approach is to constrain the dynamics such that the disturbance enters the equations in an additive manner, which motivated (7.1) in Constraint $C_{1}$.

The next example again has the structure in Figure 7.1, but we set the disturbance $d_{2}[k]=0$ in this case, i.e., there is no disturbance $D_{2}$. The dynamics are as follows:

$$
\begin{aligned}
& x_{1}[k+1]=x_{1}[k]+x_{3}^{2}[k]+x_{4}[k]+2 u_{5}[k]+d_{1}[k] \\
& x_{2}[k+1]=-x_{3}[k]+2 u_{6}[k] \\
& x_{3}[k+1]=x_{1}[k]+x_{2}[k] x_{3}[k] \\
& x_{4}[k+1]=3 x_{1}[k] .
\end{aligned}
$$

As before, we can determine control law $u_{5}[k]$ :

$$
u_{5}[k]=\left(v_{5}[k]-3 x_{1}[k]-3 x_{4}[k] x_{3}^{2}[k]\right) / 6 .
$$

For control law $u_{6}[k]$, we have

$$
\begin{aligned}
x_{3}[k+2] & =x_{1}[k+1]+x_{2}[k+1] x_{3}[k+1] \\
& =x_{1}[k]+x_{4}[k]+x_{3}^{2}[k]+2 u_{5}[k]-x_{1}[k] x_{3}[k]-x_{3}^{2}[k] x_{2}[k]
\end{aligned}
$$



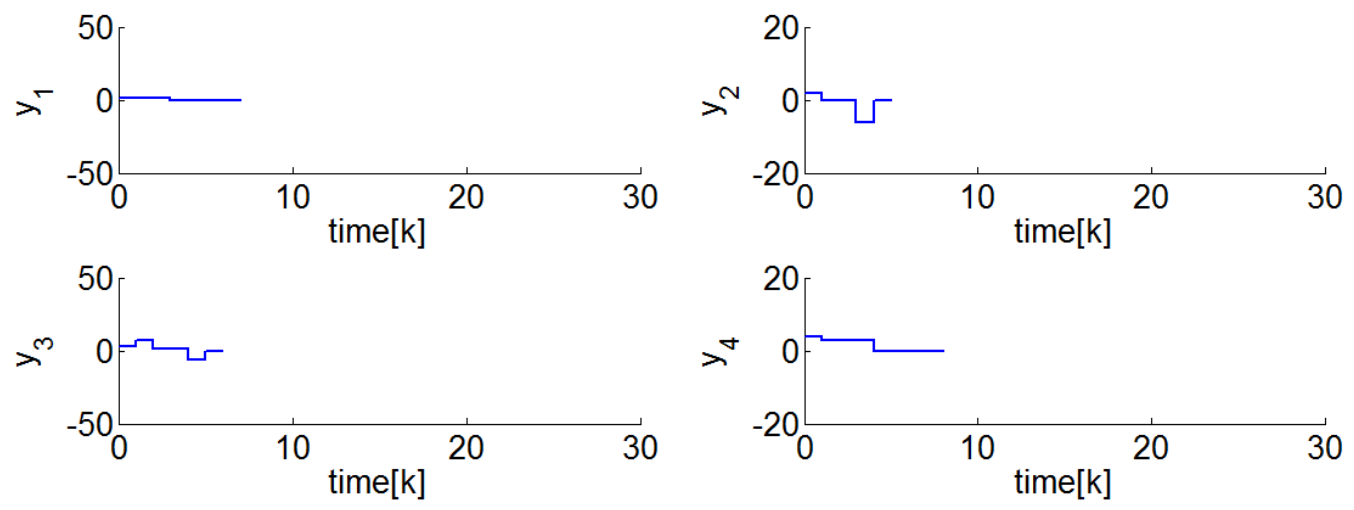

Figure 7.3: Simulation result for the queue plant with dynamics in (7.19)-(7.22). The simulation was aborted when $u_{6}[k]$ could not be computed.

$$
\begin{aligned}
& +\left(2 x_{1}[k]+2 x_{3}[k] x_{2}[k]\right) u_{6}[k] \\
= & v_{6}[k],
\end{aligned}
$$

which implies

$$
u_{6}[k]=\left(v_{6}[k]-v_{5}[k] / 3+x_{1}[k] x_{3}[k]+x_{2}[k] x_{3}^{2}[k]\right) /\left(2 x_{1}[k]+2 x_{2}[k] x_{3}[k]\right) .
$$

From (7.24), we see that the target output does not have the form of $(7.3)$ due to the statedependent term $\left(2 x_{1}[k]+2 x_{3}[k] x_{2}[k]\right) u_{6}[k]$. This term causes problems in $u_{6}[k]$ if the denominator $2 x_{1}[k]+2 x_{2}[k] x_{3}[k]$ is ever zero (or even very small). Indeed, because of this term the whole algorithm can fail, as shown in Figure 7.3. To avoid such negative effects, we impose the form of (7.3) in Constraint $C_{2}$.

Finally, consider a third example (again with the structure in Figure 7.1):

$$
\begin{aligned}
& x_{1}[k+1]=x_{1}[k]+x_{4}[k]+x_{3}^{2}[k]+2 u_{5}[k]+d_{1}[k] \\
& x_{2}[k+1]=-x_{3}[k]+u_{6}[k]+d_{2}[k] \\
& x_{3}[k+1]=x_{1}^{2}[k] x_{3}[k]+x_{2}[k] \\
& x_{4}[k+1]=3 x_{1}[k] .
\end{aligned}
$$

To find the control laws, we have

$$
\begin{aligned}
x_{4}[k+2] & =3 x_{1}[k+1] \\
& =3 x_{1}[k]+3 x_{4}[k]+3 x_{3}^{2}[k]+6 u_{5}[k] \\
& =v_{5}[k]
\end{aligned}
$$



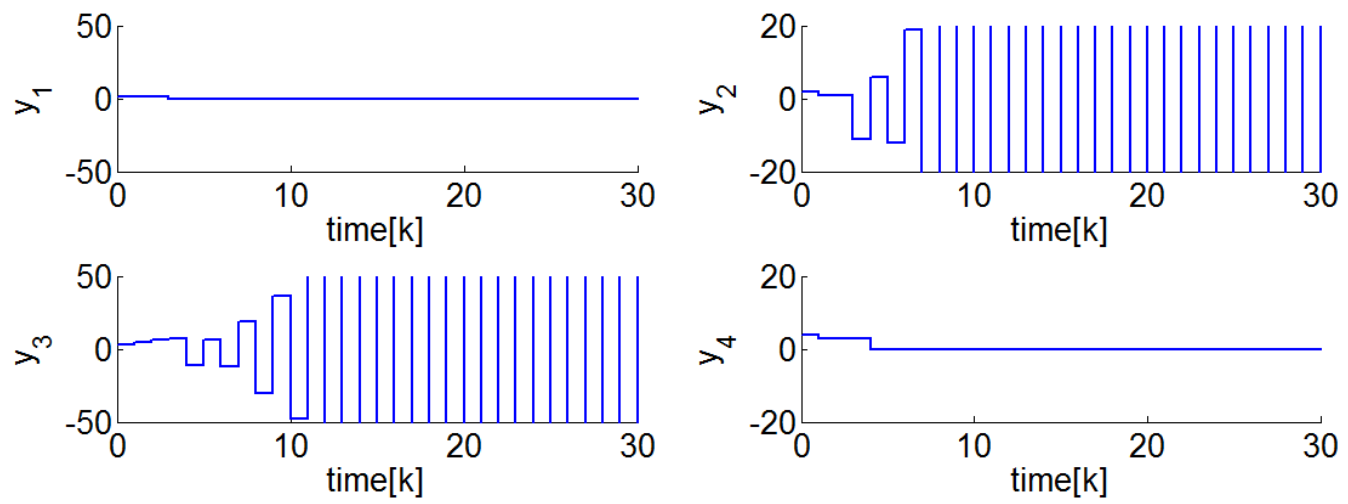

Figure 7.4: Simulation result for the queue plant with dynamics in (7.26)-(7.29).

$$
\begin{aligned}
x_{3}[k+2]= & x_{1}^{2}[k+1] x_{3}[k+1]+x_{2}[k+1] \\
= & \left(x_{1}^{2}[k] x_{3}[k]+x_{2}[k]\right)\left(x_{1}[k]+x_{4}[k]+x_{3}^{2}[k]+2 u_{5}[k]\right)^{2} \\
& -x_{3}[k]+u_{6}[k] \\
= & v_{6}[k] .
\end{aligned}
$$

Deduct the control laws

$$
\begin{aligned}
u_{5}[k] & =\left(v_{5}[k]-3 x_{1}[k]-3 x_{4}[k]-3 x_{3}^{2}[k]\right) / 6 \\
u_{6}[k] & =\left(v_{6}[k]+x_{3}[k]-\left(x_{1}^{2}[k] x_{3}[k]+x_{2}[k]\right)\left(x_{1}[k]+x_{4}[k]+x_{3}^{2}[k]+2 u_{5}[k]\right)^{2}\right),
\end{aligned}
$$

and substitute into the target output expressions:

$$
\begin{aligned}
y_{4}^{\text {tar }}[k+2] & =v_{5}[k]+3 d_{1}[k] \\
y_{3}^{\text {tar }}[k+2] & =\left(x_{1}^{2}[k] x_{3}[k]+x_{2}[k]\right)\left(2 v_{5}[k] d_{1}[k] / 3+d_{1}^{2}[k]\right)+v_{6}[k]+d_{2}[k] .
\end{aligned}
$$

Note that 7.33 violates the form in $(7.8)$ because of the term of $x_{1}^{2}[k] x_{3}[k]+x_{2}[k]$. This term makes $y_{3}^{\text {tar }}[\cdot]$ change with the state value of $x_{1}[k], x_{2}[k]$ and $x_{3}[k]$, which can have serious (destabilizing) effects. See Figure 7.4. This example motivates why we impose the form $(7.8)$ in Constraint $C_{3}$.

\subsection{A Conjecture}

Under Constraints $C_{1}-C_{3}$, we suspect that the control strategy from Chapter 5 should work. Formally, we state the following conjecture: 
Conjecture: If a nonlinear multi-agent system with constant disturbances satisfies Constraints $C_{1}-C_{3}$, the control algorithm from Chapter 5 achieves deadbeat disturbance rejection.

The conjecture has been verified to hold for many nonlinear examples that satisfy Constraints $C_{1}-C_{3}$. Here we present three such examples. For simplicity, the propagation time from any agent to any neighbour in the three examples is one. We also set the output of every plant agent as $y_{i}[k]=x_{i}[k]$.

The structure of the first system is the same shown in Figure 7.1. The dynamics satisfy Constraint $C_{1}$ :

$$
\begin{aligned}
x_{1}[k+1] & =x_{1}[k]+x_{4}[k]+x_{3}^{2}[k]+2 u_{5}[k]+d_{1}[k] \\
x_{2}[k+1] & =-x_{3}[k]+2 u_{6}[k]+d_{2}[k] \\
x_{3}[k+1] & =x_{1}^{2}[k]+4 x_{2}[k] \\
x_{4}[k+1] & =3 x_{1}[k] .
\end{aligned}
$$

Disregarding the disturbances, we have

$$
\begin{aligned}
x_{4}[k+2] & =3 x_{1}[k+1] \\
& =3 x_{1}[k]+3 x_{4}[k]+6 u_{5}[k]+3 x_{3}^{2}[k] \\
& =v_{5}[k] \\
x_{3}[k+2] & =x_{1}^{2}[k+1]+4 x_{2}[k+1] \\
& =\left(x_{1}[k]+x_{4}[k]+x_{3}^{2}[k]+2 u_{5}[k]\right)^{2}-4 x_{3}[k]+8 u_{6}[k] \\
& =v_{6}[k],
\end{aligned}
$$

which means

$$
\begin{aligned}
& u_{5}[k]=\left(v_{5}[k]-3 x_{1}[k]-3 x_{4}[k]-3 x_{3}^{2}[k]\right) / 6 \\
& u_{6}[k]=\left(v_{6}[k]-\left(x_{1}[k]+x_{4}[k]+x_{3}^{2}[k]+2 u_{5}[k]\right)^{2}+4 x_{3}[k]\right) / 8 .
\end{aligned}
$$

Substitute the two expressions of $u_{5}[k]$ and $u_{6}[k]$ into the output of the targets:

$$
\begin{aligned}
y_{4}^{\text {tar }}[k+2] & =3 x_{1}[k]+3 x_{4}[k]+6 u_{5}[k]+3 x_{3}^{2}[k]+3 d_{1}[k] \\
& =v_{5}[k]+3 d_{1}[k] \\
y_{3}^{\text {tar }}[k+2] & =\left(x_{1}[k]+x_{4}[k]+x_{3}^{2}[k]+2 u_{5}[k]+d_{1}[k]\right)^{2}-4 x_{3}[k]+8 u_{6}[k]+4 d_{2}[k] \\
& =v_{6}[k]+2 v_{5}[k] d_{1}[k] / 3+d_{1}^{2}[k]+4 d_{2}[k] .
\end{aligned}
$$

We see that (7.40) and (7.42) satisfy the form of (7.3), while (7.41) and (7.43) have the form of (7.8). So we expect that the proposed control algorithm should work for this system. Just like the linear case, form the matrices of $K_{1}$ and $K_{2}$ based on the fact that the propagation time from both control agents to their targets is two time steps:

$$
K_{1}=\left[\begin{array}{llll}
1 & 1 & 0 & 0 \\
0 & 0 & 1 & 1
\end{array}\right], K_{2}=\left[\begin{array}{ll}
1 & 0 \\
0 & 1
\end{array}\right] .
$$


Then the control laws $v[k]$ are

$$
\begin{aligned}
& v_{5}[k]=-\bar{x}_{1}[k]-\bar{x}_{2}[k]-w_{1}[k] \\
& v_{6}[k]=-\bar{x}_{3}[k]-\bar{x}_{4}[k]-w_{2}[k] .
\end{aligned}
$$

The final (nonlinear) control laws of $u[k]$ are as the following

$$
\begin{aligned}
u_{5}[k]= & \left(v_{5}[k]-3 x_{1}[k]-3 x_{4}[k]-3 x_{3}^{2}[k]\right) / 6 \\
= & \left(-\bar{x}_{1}[k]-\bar{x}_{2}[k]-w_{1}[k]-3 x_{1}[k]-3 x_{4}[k]-3 x_{3}^{2}[k]\right) / 6 \\
u_{6}[k]= & \left(v_{6}[k]-\left(x_{1}[k]+x_{4}[k]+x_{3}^{2}[k]+2 u_{5}[k]\right)^{2}+4 x_{3}[k]\right) / 8 \\
= & \left(-\bar{x}_{3}[k]-\bar{x}_{4}[k]-w_{2}[k]-\left(x_{1}[k]+x_{4}[k]\right.\right. \\
& \left.\left.+x_{3}^{2}[k]+2 u_{5}[k]\right)^{2}+4 x_{3}[k]\right) / 8,
\end{aligned}
$$

where

$$
\bar{x}[k]=\left[v_{5}[k-2], v_{5}[k-1], v_{6}[k-2], v_{6}[k-1]\right]^{T} .
$$

Set the initial conditions and disturbances as the following:

$$
\begin{aligned}
& x[k]=\left[\begin{array}{lll}
1 & 2 & 3
\end{array}\right]^{T} \\
& \bar{x}[k]=\left[\begin{array}{llll}
0 & 0 & 0 & 0
\end{array}\right]^{T} \\
& w[k]=\left[\begin{array}{ll}
0 & 0
\end{array}\right]^{T} \\
& d_{1}[k]=1(\text { for } k \geq 0) \\
& d_{2}[k]=1(\text { for } k \geq 0) \text {. }
\end{aligned}
$$

The simulation results in Figure 7.5 show that the algorithm works for this nonlinear system. It achieves deadbeat disturbance rejection in seven time steps.

The second example is the wheel-structured system shown in Figure 7.6. The dynamics of this system have the form of 7.1 :

$$
\begin{aligned}
& x_{1}[k+1]=x_{1}^{2}[k]+x_{4}[k]+x_{2}^{2}[k] x_{3}[k]+u_{6}[k]+d_{1}[k] \\
& x_{2}[k+1]=x_{1}[k] x_{3}[k]+x_{4}[k] x_{5}[k]+x_{2}^{2}[k]+u_{7}[k]+d_{2}[k] \\
& x_{3}[k+1]=\sin \left(x_{1}[k]\right)+x_{2}[k]+x_{5}[k]+u_{8}[k] \\
& x_{4}[k+1]=x_{1}[k]+x_{2}^{2}[k]+x_{4}[k] x_{5}[k] \\
& x_{5}[k+1]=x_{2}^{2}[k]+x_{4}[k]+x_{3}[k] .
\end{aligned}
$$



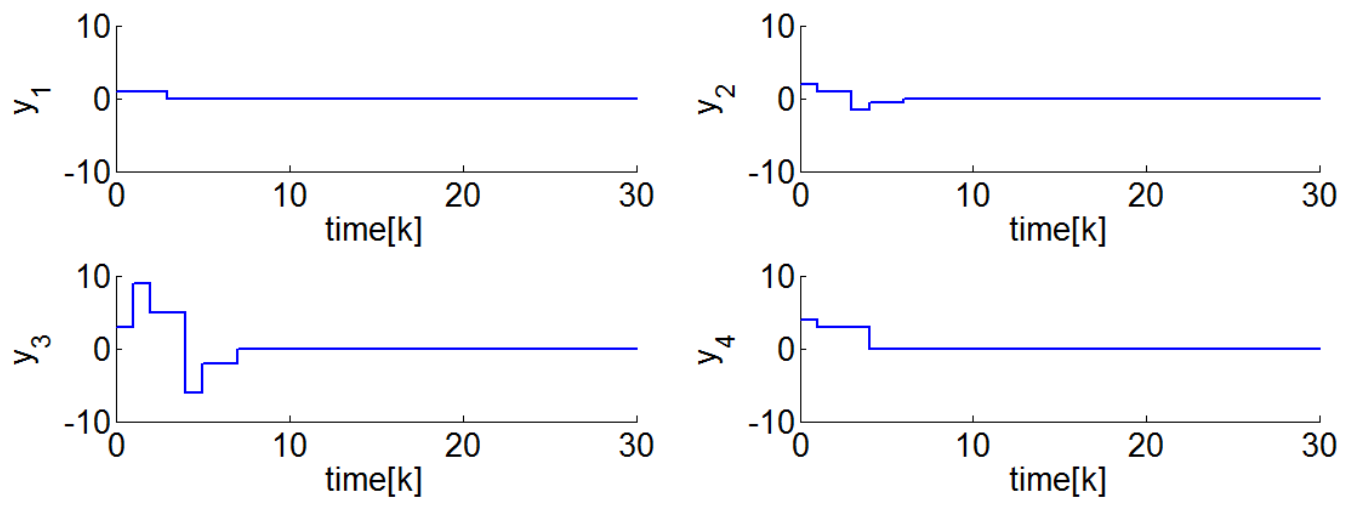

Figure 7.5: Simulation result for the queue plant with dynamics in (7.34)-7.37).

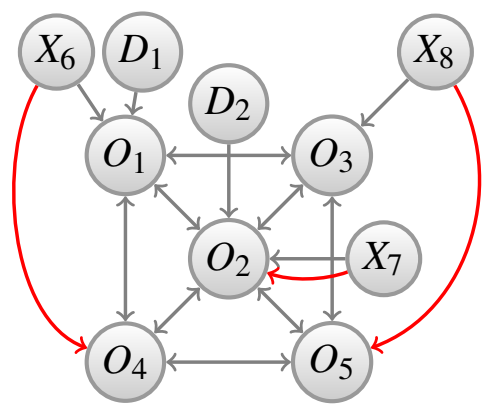

Figure 7.6: The wheel structured nonlinear system with five plant agents and two disturbance agents. 
To determine the formula of the control laws of $u[k]$, we evaluate

$$
\begin{aligned}
x_{2}[k+1]= & x_{1}[k] x_{3}[k]+x_{4}[k] x_{5}[k]+x_{2}^{2}[k]+u_{7}[k] \\
= & v_{7}[k] \\
x_{5}[k+2]= & x_{2}^{2}[k+1]+x_{3}[k+1]+x_{4}[k+1] \\
= & \left(x_{1}[k] x_{3}[k]+x_{4}[k] x_{5}[k]+x_{2}^{2}[k]+u_{7}[k]\right)^{2}+\sin \left(x_{1}[k]\right) \\
& +x_{2}[k]+x_{5}[k]+u_{8}[k]+x_{1}[k]+x_{2}^{2}[k]+x_{4}[k] x_{5}[k] \\
= & v_{8}[k] \\
x_{4}[k+2]= & x_{1}[k+1]+x_{2}^{2}[k+1]+x_{4}[k+1] x_{5}[k+1] \\
= & x_{1}^{2}[k]+x_{4}[k]+x_{2}^{2}[k] x_{3}[k]+u_{6}[k]+\left(x_{1}[k] x_{3}[k]\right. \\
& \left.+x_{4}[k] x_{5}[k]+x_{2}^{2}[k]+u_{7}[k]\right)^{2}+\left(x_{1}[k]+x_{2}^{2}[k]+x_{4}[k] x_{5}[k]\right) \\
& \left(x_{2}^{2}[k]+x_{4}[k]+x_{3}[k]\right) \\
= & v_{6}[k],
\end{aligned}
$$

which leads to

$$
\begin{aligned}
u_{7}[k]= & v_{7}[k]-x_{1}[k] x_{3}[k]-x_{4}[k] x_{5}[k]-x_{2}^{2}[k] \\
u_{8}[k]= & v_{8}[k]-\left(x_{1}[k] x_{3}[k]+x_{4}[k] x_{5}[k]+x_{2}^{2}[k]+u_{7}[k]\right)^{2}-\sin \left(x_{1}[k]\right) \\
& -x_{2}[k]-x_{5}[k]-x_{1}[k]-x_{2}^{2}[k]-x_{4}[k] x_{5}[k] \\
u_{6}[k]= & v_{6}[k]-x_{1}^{2}[k]-x_{4}[k]-x_{2}^{2}[k] x_{3}[k]-\left(x_{1}[k] x_{3}[k]\right. \\
& \left.+x_{4}[k] x_{5}[k]+x_{2}^{2}[k]+u_{7}[k]\right)^{2}-\left(x_{1}[k]+x_{2}^{2}[k]+x_{4}[k] x_{5}[k]\right) \\
& \left(x_{2}^{2}[k]+x_{4}[k]+x_{3}[k]\right) .
\end{aligned}
$$

Substitute the control laws back into the expressions for the target outputs:

$$
\begin{aligned}
y_{2}^{\text {tar }}[k+1]= & x_{1}[k] x_{3}[k]+x_{4}[k] x_{5}[k]+x_{2}^{2}[k]+u_{7}[k]+d_{2}[k] \\
= & v_{7}[k]+d_{2}[k] \\
y_{5}^{\text {tar }}[k+2]= & \left(x_{1}[k] x_{3}[k]+x_{4}[k] x_{5}[k]+x_{2}^{2}[k]+u_{7}[k]+d_{2}[k]\right)^{2} \\
& +\sin \left(x_{1}[k]\right)+x_{2}[k]+x_{5}[k]+u_{8}[k]+x_{1}[k]+x_{2}^{2}[k]+x_{4}[k] x_{5}[k] \\
= & 2 v_{7}[k] d_{2}[k]+d_{2}^{2}[k]+v_{8}[k] \\
y_{4}^{\text {tar }}[k+2]= & x_{1}^{2}[k]+x_{4}[k]+x_{2}^{2}[k] x_{3}[k]+u_{6}[k]+d_{1}[k]+\left(x_{1}[k] x_{3}[k]\right. \\
& \left.+x_{4}[k] x_{5}[k]+x_{2}^{2}[k]+u_{7}[k]+d_{2}[k]\right)^{2}+\left(x_{1}[k]+x_{2}^{2}[k]\right. \\
& \left.+x_{4}[k] x_{5}[k]\right)\left(x_{2}^{2}[k]+x_{4}[k]+x_{3}[k]\right) \\
= & v_{6}[k]+d_{1}[k]+2 v_{7}[k] d_{2}[k]+d_{2}^{2}[k],
\end{aligned}
$$

from which we confirm that the form of the target outputs are consistent with (7.3) and (7.8). As the system satisfies Constraints $C_{1}-C_{3}$, the proposed control algorithm should work for the deadbeat disturbance rejection problem. 
As the propagation time from the control agents to their targets are

$$
\begin{aligned}
& \Delta\left(X_{6}, O_{4}\right)=2 \\
& \Delta\left(X_{7}, O_{2}\right)=1 \\
& \Delta\left(X_{8}, O_{5}\right)=2
\end{aligned}
$$

it is easy to form the matrices $K_{1}$ and $K_{2}$

$$
K_{1}=\left[\begin{array}{lllll}
1 & 1 & 0 & 0 & 0 \\
0 & 0 & 1 & 0 & 0 \\
0 & 0 & 0 & 1 & 1
\end{array}\right], K_{2}=\left[\begin{array}{lll}
1 & 0 & 0 \\
0 & 1 & 0 \\
0 & 0 & 1
\end{array}\right]
$$

and the control laws of $v[k]$

$$
\begin{aligned}
& v_{6}[k]=-\bar{x}_{1}[k]-\bar{x}_{2}[k]-w_{1}[k] \\
& v_{7}[k]=-\bar{x}_{3}[k]-w_{2}[k] \\
& v_{8}[k]=-\bar{x}_{4}[k]-\bar{x}_{5}[k]-w_{3}[k] .
\end{aligned}
$$

Thus, the final control laws $u[k]$ are

$$
\begin{aligned}
u_{7}[k]= & v_{7}[k]-x_{1}[k] x_{3}[k]-x_{4}[k] x_{5}[k]-x_{2}^{2}[k] \\
= & -\bar{x}_{3}[k]-w_{2}[k]-x_{1}[k] x_{3}[k]-x_{4}[k] x_{5}[k]-x_{2}^{2}[k] \\
u_{8}[k]= & v_{8}[k]-\left(x_{1}[k] x_{3}[k]+x_{4}[k] x_{5}[k]+x_{2}^{2}[k]+u_{7}[k]\right)^{2}-\sin \left(x_{1}[k]\right) \\
& -x_{2}[k]-x_{5}[k]-x_{1}[k]-x_{2}^{2}[k]-x_{4}[k] x_{5}[k] \\
= & -\bar{x}_{4}[k]-\bar{x}_{5}[k]-w_{3}[k]-\left(x_{1}[k] x_{3}[k]+x_{4}[k] x_{5}[k]+x_{2}^{2}[k]\right. \\
& \left.+u_{7}[k]\right)^{2}-\sin \left(x_{1}[k]\right)-x_{2}[k]-x_{5}[k]-x_{1}[k]-x_{2}^{2}[k]-x_{4}[k] x_{5}[k] \\
u_{6}[k]= & v_{6}[k]-x_{1}^{2}[k]-x_{4}[k]-x_{2}^{2}[k] x_{3}[k]-\left(x_{1}[k] x_{3}[k]\right. \\
& \left.+x_{4}[k] x_{5}[k]+x_{2}^{2}[k]+u_{7}[k]\right)^{2}-x_{1}[k]-x_{2}^{2}[k]-x_{4}[k] x_{5}[k] \\
& -x_{2}^{2}[k]-x_{4}[k]-x_{3}[k] \\
= & -\bar{x}_{1}[k]-\bar{x}_{2}[k]-w_{1}[k]-x_{1}^{2}[k]-x_{4}[k]-x_{2}^{2}[k] x_{3}[k] \\
& -\left(x_{1}[k] x_{3}[k]+x_{4}[k] x_{5}[k]+x_{2}^{2}[k]+u_{7}[k]\right)^{2}-\left(x_{1}[k]+x_{2}^{2}[k]\right. \\
& \left.+x_{4}[k] x_{5}[k]\right)\left(x_{2}^{2}[k]+x_{4}[k]+x_{3}[k]\right),
\end{aligned}
$$

where

$$
\bar{x}[k]=\left[v_{6}[k-2], v_{6}[k-1], v_{7}[k-1], v_{8}[k-2], v_{8}[k-1]\right]^{T} .
$$



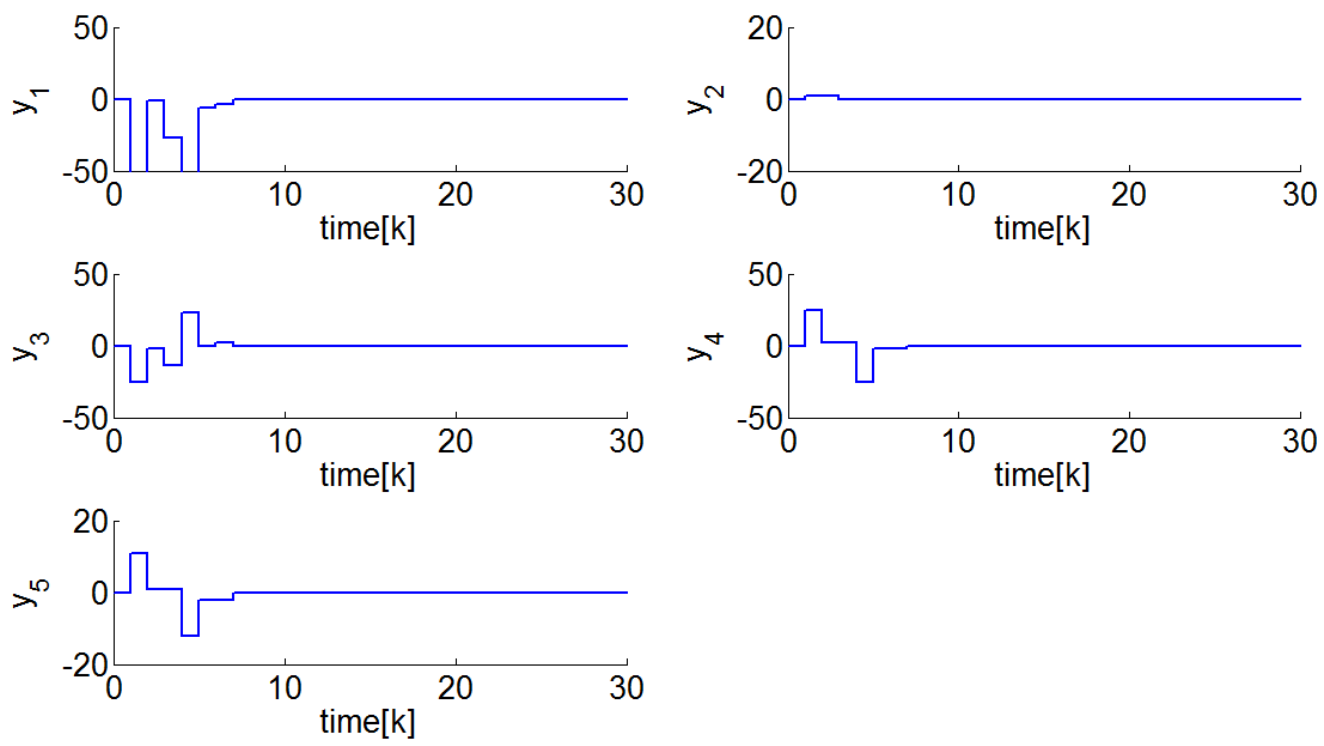

Figure 7.7: Simulation result for the wheel plant with dynamics in (7.46) 7.49 .

Set the initial conditions as the following:

$$
\begin{aligned}
x[k] & =\left[\begin{array}{lllll}
1 & 2 & 3 & 4 & 5
\end{array}\right]^{T} \\
\bar{x}[k] & =\left[\begin{array}{lllll}
0 & 0 & 0 & 0 & 0
\end{array}\right]^{T} \\
w[k] & =\left[\begin{array}{lll}
0 & 0 & 0
\end{array}\right]^{T} \\
d_{1}[k] & =1(\text { for } k \geq 0) \\
d_{2}[k] & =1(\text { for } k \geq 0) .
\end{aligned}
$$

Deadbeat disturbance rejection is achieved, as shown in the simulation results in Figure 7.7

The last example is the grid-structured nonlinear system shown in Figure 7.8. The dynamics of this system are

$$
\begin{aligned}
x_{1}[k+1] & =x_{1}^{2}[k]+x_{7}[k]+\sin \left(x_{4}[k]\right)+x_{4}[k] x_{5}[k]+u_{12}[k]+d_{1}[k] \\
x_{2}[k+1] & =\left(x_{4}[k]+x_{5}[k]+x_{8}[k]\right)^{3}+u_{10}[k]+d_{2}[k] \\
x_{3}[k+1] & =\sin \left(x_{7}[k]\right) \cos \left(x_{5}[k]\right)+x_{3}[k]+x_{6}^{2}[k]+u_{14}[k]+d_{3}[k] \\
x_{4}[k+1] & =x_{1}[k]+x_{7}[k]+x_{5}[k]+x_{4}^{2}[k]+x_{8}^{2}[k]+x_{2}^{2}[k]+u_{11}[k] \\
x_{5}[k+1] & =x_{1}[k]+x_{2}[k]+x_{3}[k]+x_{4}[k]+x_{6}[k]+x_{7}[k]+x_{8}[k]+x_{9}[k] \\
x_{6}[k+1] & =x_{3}[k]+x_{5}[k]+x_{7}[k]+x_{8}[k]+x_{9}[k] \\
x_{7}[k+1] & =x_{1}[k] x_{3}[k]+x_{4}^{2}[k]+x_{5}[k]+x_{6}[k]+u_{13}[k]
\end{aligned}
$$




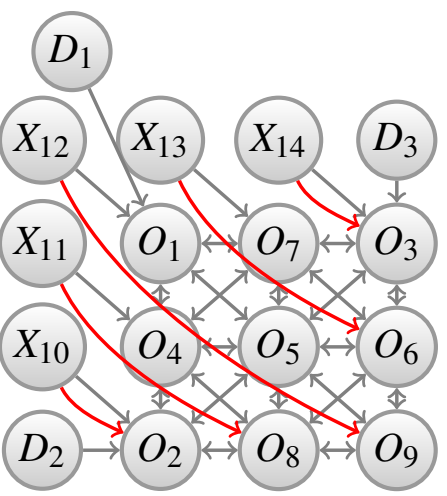

Figure 7.8: The grid structured nonlinear system with nine plant agents and three disturbance agents.

$$
\begin{aligned}
x_{8}[k+1] & =x_{2}[k]+x_{4}[k]+x_{5}[k]+x_{6}[k]+x_{9}[k] \\
x_{9}[k+1] & =x_{5}[k]+x_{6}[k]+x_{8}[k] .
\end{aligned}
$$

Determine the control laws $u[k]$ by ignoring the disturbances,

$$
\begin{aligned}
x_{2}[k+1]= & \left(x_{4}[k]+x_{5}[k]+x_{8}[k]\right)^{3}+u_{10}[k] \\
= & v_{10}[k] \\
x_{3}[k+1]= & \sin \left(x_{7}[k]\right) \cos \left(x_{5}[k]\right)+x_{3}[k]+x_{6}^{2}[k]+u_{14}[k] \\
= & v_{14}[k] \\
x_{8}[k+2]= & x_{2}[k+1]+x_{4}[k+1]+x_{5}[k+1]+x_{6}[k+1]+x_{9}[k+1] \\
= & 2 x_{1}[k]+3 x_{7}[k]+2 x_{3}[k]+x_{4}[k]+x_{4}^{2}[k]+3 x_{5}[k]+2 x_{6}[k] \\
& +x_{2}[k]+x_{2}^{2}[k]+3 x_{8}[k]+x_{8}^{2}[k]+2 x_{9}[k]+\left(x_{4}[k]+x_{5}[k]+x_{8}[k]\right)^{3} \\
& +u_{10}[k]+u_{11}[k] \\
= & v_{11}[k] \\
x_{6}[k+2]= & x_{3}[k+1]+x_{5}[k+1]+x_{7}[k+1]+x_{8}[k+1]+x_{9}[k+1] \\
= & x_{1}[k]+x_{1}[k] x_{3}[k]+x_{7}[k]+\sin \left(x_{7}[k]\right) \cos \left(x_{5}[k]\right)+2 x_{3}[k]+2 x_{4}[k] \\
& +x_{4}^{2}[k]+3 x_{5}[k]+4 x_{6}[k]+x_{6}^{2}[k]+2 x_{2}[k]+2 x_{8}[k]+2 x_{9}[k] \\
& +u_{14}[k]+u_{13}[k] \\
= & x_{5}[k+2]+x_{6}[k+2]+x_{8}[k+2] \\
= & x_{1}[k+1]+2 x_{7}[k+1]+2 x_{3}[k+1]+2 x_{4}[k+1]+2 x_{5}[k+1]+2 x_{6}[k+1] \\
& +2 x_{2}[k+1]+2 x_{8}[k+1]+3 x_{9}[k+1] \\
= & 4 x_{1}[k]+x_{1}^{2}[k]+2 x_{1}[k] x_{3}[k]+7 x_{7}[k]+2 \sin \left(x_{7}[k]\right) \cos \left(x_{5}[k]\right)+6 x_{3}[k] \\
& +4 x_{4}[k]+4 x_{4}^{2}[k]+\sin \left(x_{4}[k]\right)+x_{4}[k] x_{5}[k]+2\left(x_{4}[k]+x_{5}[k]+x_{8}[k]\right)^{3} \\
x_{9}[k] &
\end{aligned}
$$




$$
\begin{aligned}
& +11 x_{5}[k]+9 x_{6}[k]+2 x_{6}^{2}[k]+4 x_{2}[k]+2 x_{2}^{2}[k]+7 x_{8}[k]+6 x_{9}[k] \\
& +2 u_{13}[k]+2 u_{14}[k]+2 u_{11}[k]+2 u_{10}[k]+u_{12}[k]
\end{aligned}
$$

and therefore deriving

$$
\begin{aligned}
u_{10}[k]= & v_{10}[k]-\left(x_{4}[k]+x_{5}[k]+x_{8}[k]\right)^{3} \\
u_{14}[k]= & v_{14}[k]-\sin \left(x_{7}[k]\right) \cos \left(x_{5}[k]\right)-x_{3}[k]-x_{6}^{2}[k] \\
u_{11}[k]= & v_{11}[k]-2 x_{1}[k]-3 x_{7}[k]-2 x_{3}[k]-x_{4}[k]-x_{4}^{2}[k] \\
& -3 x_{5}[k]-2 x_{6}[k]-x_{2}[k]-x_{2}^{2}[k]-3 x_{8}[k]-x_{8}^{2}[k]-2 x_{9}[k] \\
& -\left(x_{4}[k]+x_{5}[k]+x_{8}[k]\right)^{3}-u_{10}[k] \\
u_{13}[k]= & v_{13}[k]-x_{1}[k]-x_{1}[k] x_{3}[k]-x_{7}[k]-\sin \left(x_{7}[k]\right) \cos \left(x_{5}[k]\right) \\
& -2 x_{3}[k]-2 x_{4}[k]-x_{4}^{2}[k]-3 x_{5}[k]-4 x_{6}[k]-x_{6}^{2}[k] \\
& -x_{2}[k]-2 x_{8}[k]-2 x_{9}[k]-u_{14}[k] \\
u_{12}[k]= & v_{12}[k]-4 x_{1}[k]-x_{1}^{2}[k]-2 x_{1}[k] x_{3}[k]-7 x_{7}[k]-2 \sin \left(x_{7}[k]\right) \cos \left(x_{5}[k]\right) \\
& -6 x_{3}[k]-4 x_{4}[k]-4 x_{4}^{2}[k]-\sin \left(x_{4}[k]\right)-x_{4}[k] x_{5}[k]-11 x_{5}[k] \\
& -2\left(x_{4}[k]+x_{5}[k]+x_{8}[k]\right)^{3}-9 x_{6}[k]-2 x_{6}^{2}[k]-4 x_{2}[k]-2 x_{2}^{2}[k] \\
& -7 x_{8}[k]-6 x_{9}[k]-2 u_{13}[k]-2 u_{14}[k]-2 u_{11}[k]-2 u_{10}[k] .
\end{aligned}
$$

Substitute the control laws $u[k]$ into the target output expressions:

$$
\begin{aligned}
y_{2}^{\text {tar }}[k+1]= & \left(x_{4}[k]+x_{5}[k]+x_{8}[k]\right)^{3}+u_{10}[k]+d_{2}[k] \\
= & v_{10}[k]+d_{2}[k] \\
y_{3}^{\text {tar }}[k+1]= & \sin \left(x_{7}[k]\right) \cos \left(x_{5}[k]\right)+x_{3}[k]+x_{6}^{2}[k]+u_{14}[k]+d_{3}[k] \\
= & v_{14}[k]+d_{3}[k] \\
y_{8}^{\text {tar }}[k+2]= & 2 x_{1}[k]+3 x_{7}[k]+2 x_{3}[k]+x_{4}[k]+x_{4}^{2}[k]+3 x_{5}[k]+2 x_{6}[k]+d_{2}[k] \\
& +x_{2}[k]+x_{2}^{2}[k]+3 x_{8}[k]+x_{8}^{2}[k]+2 x_{9}[k]+\left(x_{4}[k]+x_{5}[k]+x_{8}[k]\right)^{3} \\
= & v_{11}[k]+d_{2}[k] \\
y_{6}^{\text {tar }}[k+2]= & x_{1}[k]+x_{1}[k] x_{3}[k]+x_{7}[k]+\sin \left(x_{7}[k]\right) \cos \left(x_{5}[k]\right)+2 x_{3}[k]+2 x_{4}[k] \\
& +x_{4}^{2}[k]+3 x_{5}[k]+4 x_{6}[k]+x_{6}^{2}[k]+2 x_{2}[k]+2 x_{8}[k]+2 x_{9}[k] \\
& +u_{14}[k]+u_{13}[k]+d_{3}[k] \\
= & v_{13}[k]+d_{3}[k] \\
y_{9}^{\text {tar }}[k+3]= & 4 x_{1}[k]+x_{1}^{2}[k]+2 x_{1}[k] x_{3}[k]+7 x_{7}[k]+2 \sin \left(x_{7}[k]\right) \cos \left(x_{5}[k]\right) \\
& +6 x_{3}[k]+4 x_{4}[k]+4 x_{4}^{2}[k]+\sin \left(x_{4}[k]\right)+x_{4}[k] x_{5}[k] \\
& +2\left(x_{4}[k]+x_{5}[k]+x_{8}[k]\right)^{3}+11 x_{5}[k]+9 x_{6}[k]+2 x_{6}^{2}[k] \\
& +4 x_{2}[k]+2 x_{2}^{2}[k]+7 x_{8}[k]+6 x_{9}[k]+2 u_{13}[k]+2 u_{14}[k] \\
& +2 u_{11}[k]+2 u_{10}[k]+u_{12}[k]+d_{1}[k]+2 d_{2}[k]+2 d_{3}[k] \\
= & v_{12}[k]+d_{1}[k]+2 d_{2}[k]+2 d_{3}[k], \\
&
\end{aligned}
$$


These have the form of $(7.3)$ and $(7.8)$. Hence, the system meets Constraints $C_{1}-C_{3}$. Lastly, we determine the control laws $v[k]$. As the propagation time from control agents to their targets are

$$
\begin{aligned}
& \Delta\left(X_{10}, O_{2}\right)=1 \\
& \Delta\left(X_{11}, O_{8}\right)=2 \\
& \Delta\left(X_{12}, O_{9}\right)=3 \\
& \Delta\left(X_{13}, O_{6}\right)=2 \\
& \Delta\left(X_{14}, O_{3}\right)=1,
\end{aligned}
$$

the matrices $K_{1}$ and $K_{2}$ are

$$
K_{1}=\left[\begin{array}{lllllllll}
1 & 0 & 0 & 0 & 0 & 0 & 0 & 0 & 0 \\
0 & 1 & 1 & 0 & 0 & 0 & 0 & 0 & 0 \\
0 & 0 & 0 & 1 & 1 & 1 & 0 & 0 & 0 \\
0 & 0 & 0 & 0 & 0 & 0 & 1 & 1 & 0 \\
0 & 0 & 0 & 0 & 0 & 0 & 0 & 0 & 1
\end{array}\right], K_{2}=\left[\begin{array}{lllll}
1 & 0 & 0 & 0 & 0 \\
0 & 1 & 0 & 0 & 0 \\
0 & 0 & 1 & 0 & 0 \\
0 & 0 & 0 & 1 & 0 \\
0 & 0 & 0 & 0 & 1
\end{array}\right] .
$$

The control laws $v[k]$ are

$$
\begin{aligned}
& v_{10}[k]=-\bar{x}_{1}[k]-w_{1}[k] \\
& v_{11}[k]=-\bar{x}_{2}[k]-\bar{x}_{3}[k]-w_{2}[k] \\
& v_{12}[k]=-\bar{x}_{4}[k]-\bar{x}_{5}[k]-\bar{x}_{6}[k]-w_{3}[k] \\
& v_{13}[k]=-\bar{x}_{7}[k]-\bar{x}_{8}[k]-w_{4}[k] \\
& v_{14}[k]=-\bar{x}_{9}[k]-w_{5}[k],
\end{aligned}
$$

and therefore the final control laws $u[k]$ are

$$
\begin{aligned}
u_{10}[k]= & -\bar{x}_{1}[k]-w_{1}[k]-\left(x_{4}[k]+x_{5}[k]+x_{8}[k]\right)^{3} \\
u_{14}[k]= & -\bar{x}_{9}[k]-w_{5}[k]-\sin \left(x_{7}[k]\right) \cos \left(x_{5}[k]\right)-x_{3}[k]-x_{6}^{2}[k] \\
u_{11}[k]= & -\bar{x}_{2}[k]-\bar{x}_{3}[k]-w_{2}[k]-2 x_{1}[k]-3 x_{7}[k]-2 x_{3}[k] \\
& -x_{4}[k]-x_{4}^{2}[k]-3 x_{5}[k]-2 x_{6}[k]-x_{2}[k]-x_{2}^{2}[k]-3 x_{8}[k] \\
& -x_{8}^{2}[k]-2 x_{9}[k]-\left(x_{4}[k]+x_{5}[k]+x_{8}[k]\right)^{3}-u_{10}[k] \\
u_{13}[k]= & -\bar{x}_{7}[k]-\bar{x}_{8}[k]-w_{4}[k]-x_{1}[k]-x_{1}[k] x_{3}[k]-x_{7}[k] \\
& -\sin \left(x_{7}[k]\right) \cos \left(x_{5}[k]\right)-2 x_{3}[k]-2 x_{4}[k]-x_{4}^{2}[k]-3 x_{5}[k]-4 x_{6}[k] \\
& -x_{6}^{2}[k]-x_{2}[k]-2 x_{8}[k]-2 x_{9}[k]-u_{14}[k] \\
u_{12}[k]= & -\bar{x}_{4}[k]-\bar{x}_{5}[k]-\bar{x}_{6}[k]-w_{3}[k]-4 x_{1}[k]-x_{1}^{2}[k]-2 x_{1}[k] x_{3}[k] \\
& -7 x_{7}[k]-2 \sin \left(x_{7}[k]\right) \cos \left(x_{5}[k]\right)-6 x_{3}[k]-4 x_{4}[k]-4 x_{4}^{2}[k]-\sin \left(x_{4}[k]\right) \\
& -x_{4}[k] x_{5}[k]-11 x_{5}[k]-2\left(x_{4}[k]+x_{5}[k]+x_{8}[k]\right)^{3}-9 x_{6}[k]-2 x_{6}^{2}[k]-4 x_{2}[k] \\
& -2 x_{2}^{2}[k]-7 x_{8}[k]-6 x_{9}[k]-2 u_{13}[k]-2 u_{14}[k]-2 u_{11}[k]-2 u_{10}[k],
\end{aligned}
$$



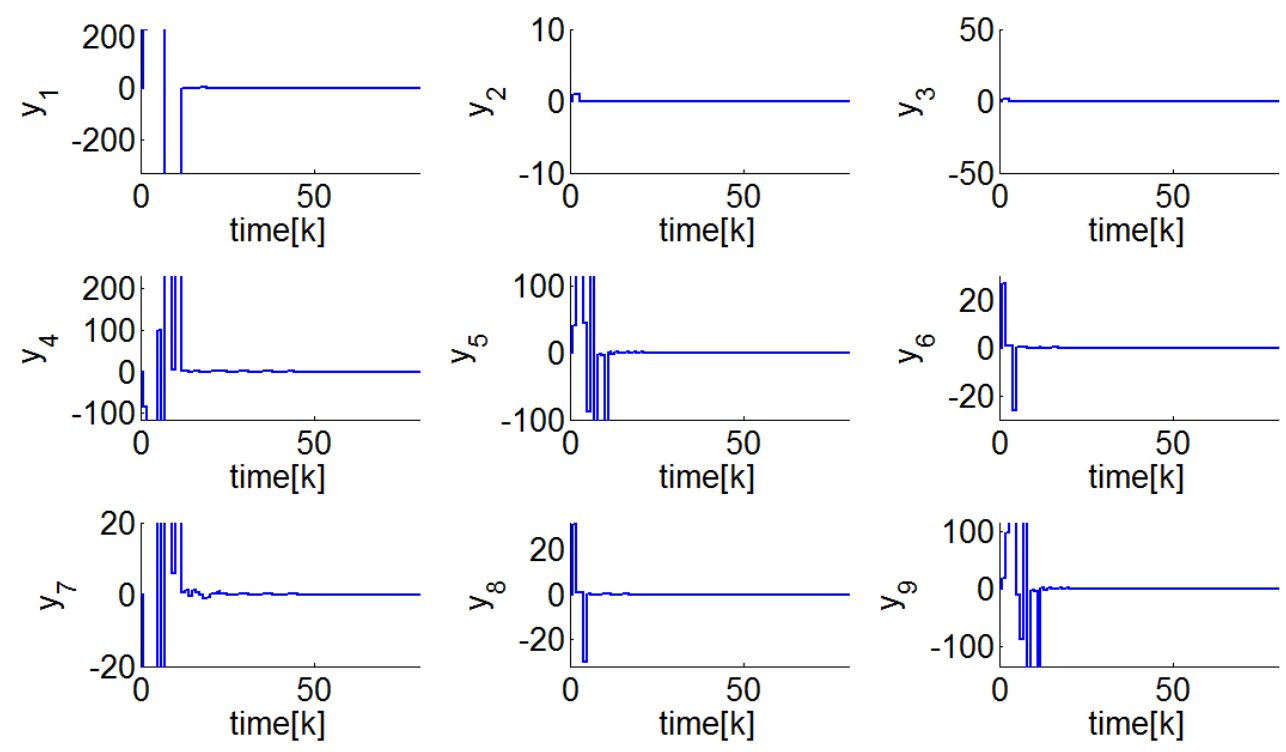

Figure 7.9: Simulation result for the wheel plant with dynamics in (7.60)-(7.68).

where

$$
\begin{array}{r}
\bar{x}[k]=\left[v_{10}[k-1], v_{11}[k-2], v_{11}[k-1], v_{12}[k-3], v_{12}[k-2],\right. \\
\left.v_{12}[k-1], v_{13}[k-2], v_{13}[k-1], v_{14}[k-1]\right]^{T} .
\end{array}
$$

Set the initial conditions and disturbances as

$$
\begin{aligned}
x[k] & =\left[\begin{array}{lllllllll}
1 & 2 & 3 & 4 & 5 & 6 & 7 & 8 & 9
\end{array}\right]^{T} \\
\bar{x}[k] & =\left[\begin{array}{lllllllll}
0 & 0 & 0 & 0 & 0 & 0 & 0 & 0 & 0
\end{array}\right]^{T} \\
w[k] & =\left[\begin{array}{lllll}
0 & 0 & 0 & 0 & 0
\end{array}\right]^{T} \\
d_{1}[k] & =1(\text { for } k \geq 0) \\
d_{2}[k] & =1(\text { for } k \geq 0) .
\end{aligned}
$$

The simulation results in Figure 7.9 shows that deadbeat disturbance rejection is achieved.

The three examples verify the conjecture that the proposed control algorithm achieves deadbeat disturbance rejection for systems that satisfy Constraints $C_{1}-C_{3}$. A formal proof is left for future research work. 


\section{Chapter 8}

\section{Summary and Future Directions}

This work was initially motivated by a psychological-control problem where some people, playing the role of control agents, attempt to control a crowd of people. Based on previous research that focused on decentralized output regulation of nonlinear discrete-time multi-agent systems, our goal was to achieve deadbeat disturbance rejection for unknown constant disturbances. Two specific problems were investigated: (1) for a given plant with known disturbance agent locations, determine how many control agents are needed, where their locations should be, and which plant agents should be chosen as their targets, in order to successfully obtain deadbeat disturbance rejection; (2) for a given plant with known disturbance agent locations and for a given set of control agent locations with known targets, find, if possible, control laws to successfully obtain deadbeat disturbance rejection.

Our overall approach is to extend research results developed for the regulation problem to the disturbance rejection problem. For Problem 1, a new necessary condition was developed for targeting and growing to succeed, namely that a control agent must be connected to each plant agent to which a disturbance agent is connected. This new condition, in addition to the four necessary conditions previously developed, puts a bound on the minimal number of control agents, and constrains the locations of control agents, needed for successful disturbance rejection. For Problem 2, a new control approach, essentially a multi-loop control scheme with an inner loop based on the regulation approach from the previous research, and an outer loop which is composed of integrators and state-feedback terms, was proposed. The approach was shown to work well for linear systems, and preliminary results show that it works for certain nonlinear systems. The proposed approach is a new way to deal with deadbeat disturbance rejection. A strength of the approach include the simple interpretation of the necessary condition in Lemma 1 , making the result useful for large-scale systems. Although not fully studied yet, another likely strength is that the approach often results in less sensing workload per control agent compared to standard centralized methods for non-trivial examples with multiple control agents. On the other hand, the approach is significantly more complicated than the regulation results. 
There are a few research directions that warrant further exploration. In the following, we have listed down some ideas for future work:

(a) Better characterize the class of nonlinear multi-agent systems for which the proposed approach works.

(b) Prove the conjecture proposed for the specified class of nonlinear system.

(c) Loosen up the requirements that disturbances be constants and that deadbeat disturbance rejection be obtained. In the linear case, it is predicted to be straightforward to loosen both of these requirements, but the nonlinear case is less obvious.

(d) Further investigate the improvement in sensing workload for the proposed technique compared to standard centralized methods.

(e) Accommodate delays and constraints on communications and sensing.

(f) Allow for unknown or time-varying locations of disturbance agents. A fault detection scheme may be useful to determine the locations of disturbance agents.

(g) Extend the work from discrete-time systems to a continuous-time framework or discreteevent framework, both which may be more natural in some applications. 


\section{Appendix A: Proof of Lemma 2}

Before proving controllability of the augmented system (5.23)-(5.24), let us recall that the augmented plant is

$$
\begin{aligned}
x^{*}[k+1] & =A_{1}^{*} x^{*}[k]+B^{*}(v[k]+\tilde{d}[k]) \\
y^{\text {tar }}[k] & =C^{*} x^{*}[k]
\end{aligned}
$$

where

$$
\begin{aligned}
x^{*} & =\left[\begin{array}{c}
\bar{x}[k] \\
w[k]
\end{array}\right], \\
A_{1}^{*} & =\left[\begin{array}{ll}
\bar{A} & O \\
\bar{C} & I
\end{array}\right], \\
B^{*} & =\left[\begin{array}{c}
\bar{B} \\
O
\end{array}\right], \\
C^{*} & =\left[\begin{array}{ll}
\bar{C} & O
\end{array}\right],
\end{aligned}
$$

$$
\begin{aligned}
\bar{A} & =\text { block } \operatorname{diag}\left\{A_{\Delta\left(X_{n+1}, T_{n+1}\right)}^{\prime}, \ldots, A_{\Delta\left(X_{n+m}, T_{n+m}\right)}^{\prime}\right\} \\
\bar{B} & =\text { block } \operatorname{diag}\left\{B_{\Delta\left(X_{n+1}, T_{n+1}\right)}^{\prime}, \ldots, B_{\Delta\left(X_{n+m}, T_{n+m}\right)}^{\prime}\right\} \\
\bar{C} & =\text { block } \operatorname{diag}\left\{C_{\Delta\left(X_{n+1}, T_{n+1}\right)}^{\prime}, \ldots, C_{\Delta\left(X_{n+m}, T_{n+m}\right)}^{\prime}\right\}
\end{aligned}
$$

and

$$
A_{r}^{\prime}=\left[\begin{array}{ccccc}
0 & 1 & 0 & \cdots & 0 \\
0 & 0 & 1 & & 0 \\
\vdots & \vdots & \ddots & \ddots & \vdots \\
0 & 0 & \cdots & 0 & 1 \\
0 & 0 & 0 & \cdots & 0
\end{array}\right] \in R^{r \times r}
$$




$$
\begin{aligned}
B_{r}^{\prime} & =\left[\begin{array}{c}
0 \\
\vdots \\
0 \\
1
\end{array}\right] \in R^{r \times 1}, \\
C_{r}^{\prime} & =\left[\begin{array}{llll}
1 & 0 & \cdots & 0
\end{array}\right] \in R^{1 \times r}, \\
D_{r}^{\prime} & =0 .
\end{aligned}
$$

Proving that the augmented system $(5.23)-(5.24)$ is controllable is equivalent to proving that matrix $\left[A_{1}^{*}-\lambda I B^{*}\right]$ is full rank, for each eigenvalue $\lambda$ of matrix $A_{1}^{*}[3]$. The following is the proof that the matrix $\left[A_{1}^{*}-\lambda I B^{*}\right]$ is full rank for each eigenvalue of matrix $A_{1}^{*}$.

First, we show the complete form of the $\left(n^{\prime}+m\right) \times\left(n^{\prime}+m\right)$ matrix of $A_{1}^{*}$, where

$$
n^{\prime}=\Delta_{1}+\Delta_{2}+\cdots+\Delta_{m}
$$


as follows:

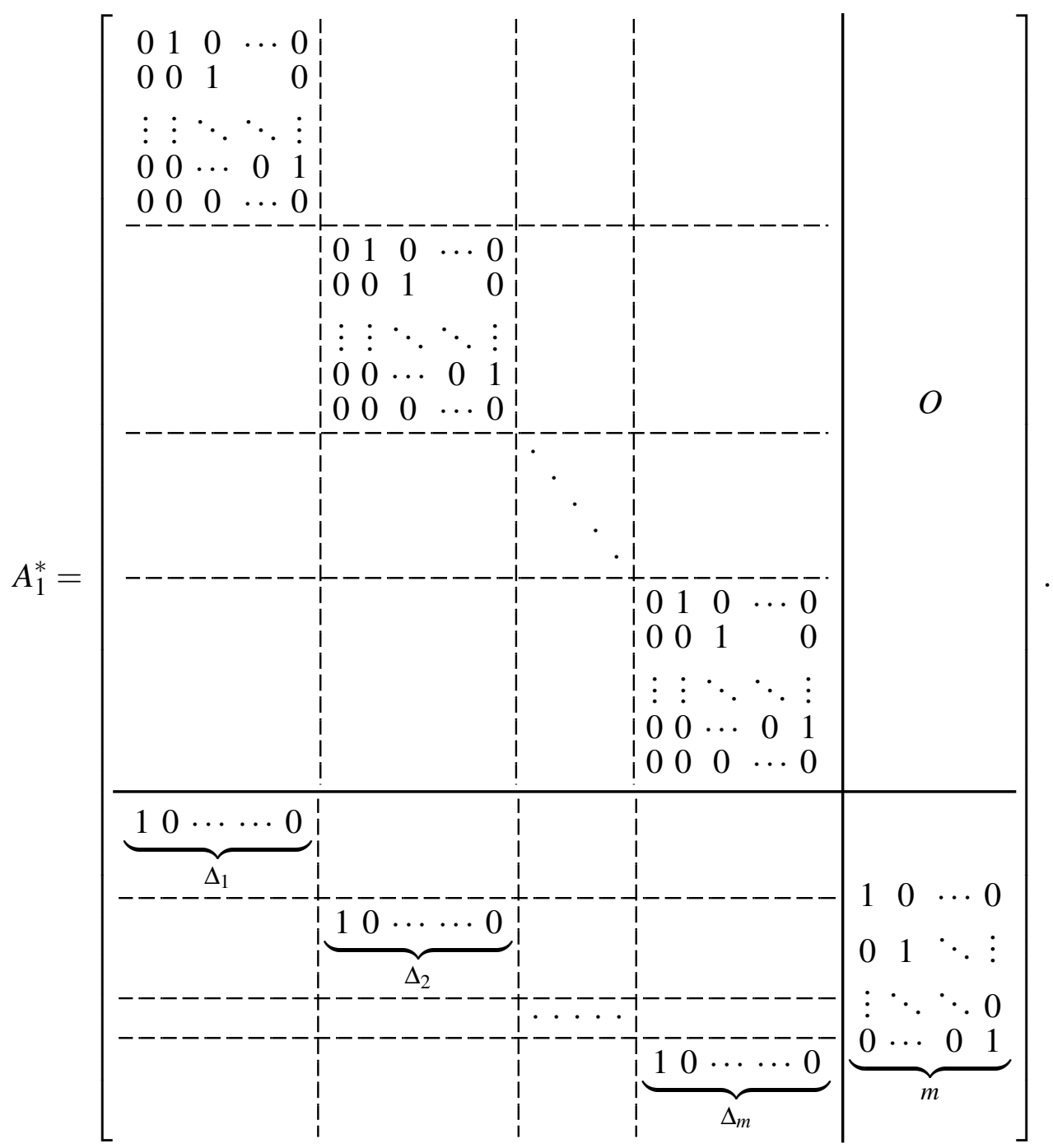

The empty blocks are all zero matrices.

To find the eigenvalues of $A_{1}^{*}$, we set

$$
\left|\lambda I-A_{1}^{*}\right|=0
$$


To this end, we have $\lambda I-A_{1}^{*}=$

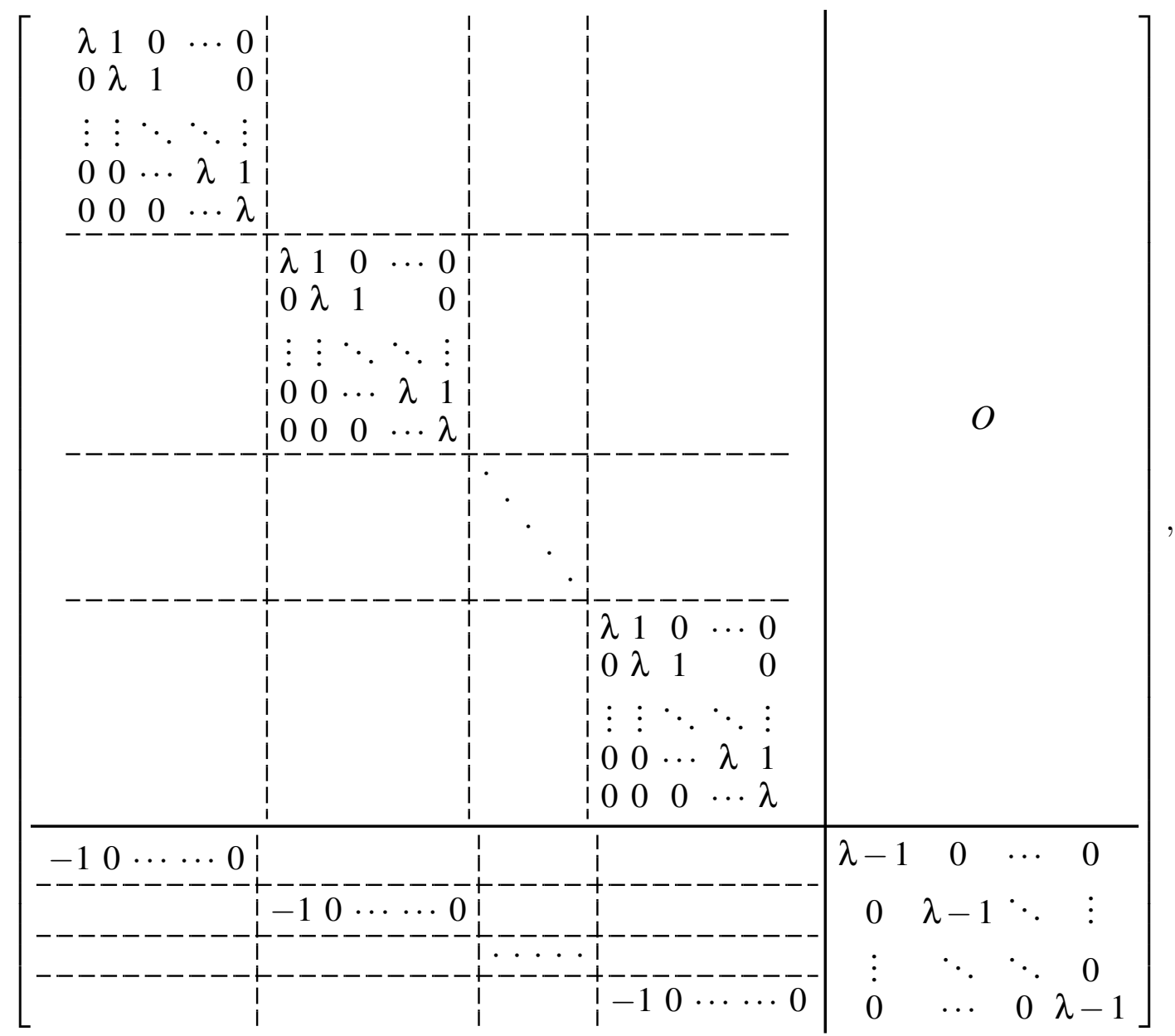

which implies that

$$
\left|\lambda I-A_{1}^{*}\right|=\lambda^{n^{\prime}} \cdot(\lambda-1)^{m}=0
$$

and therefore

$$
\lambda=0 \text { or } 1 .
$$


When $\lambda=0$, we have $\left[\begin{array}{lll}A_{1}^{*}-0 \cdot I & B^{*}\end{array}\right]=$

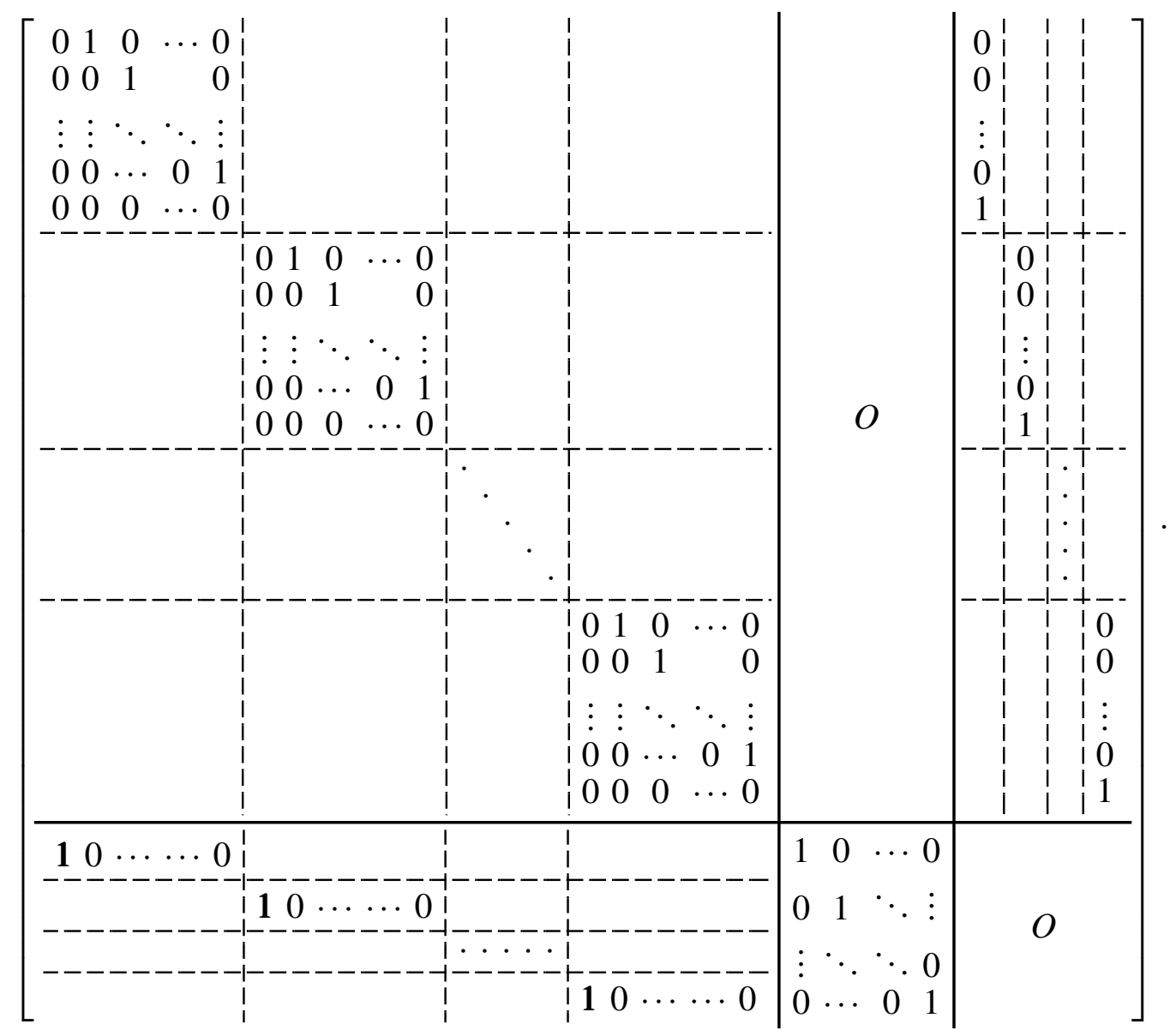

After some elementary row/column operations, the bold 1's can be cancelled. The matrix be- 
comes

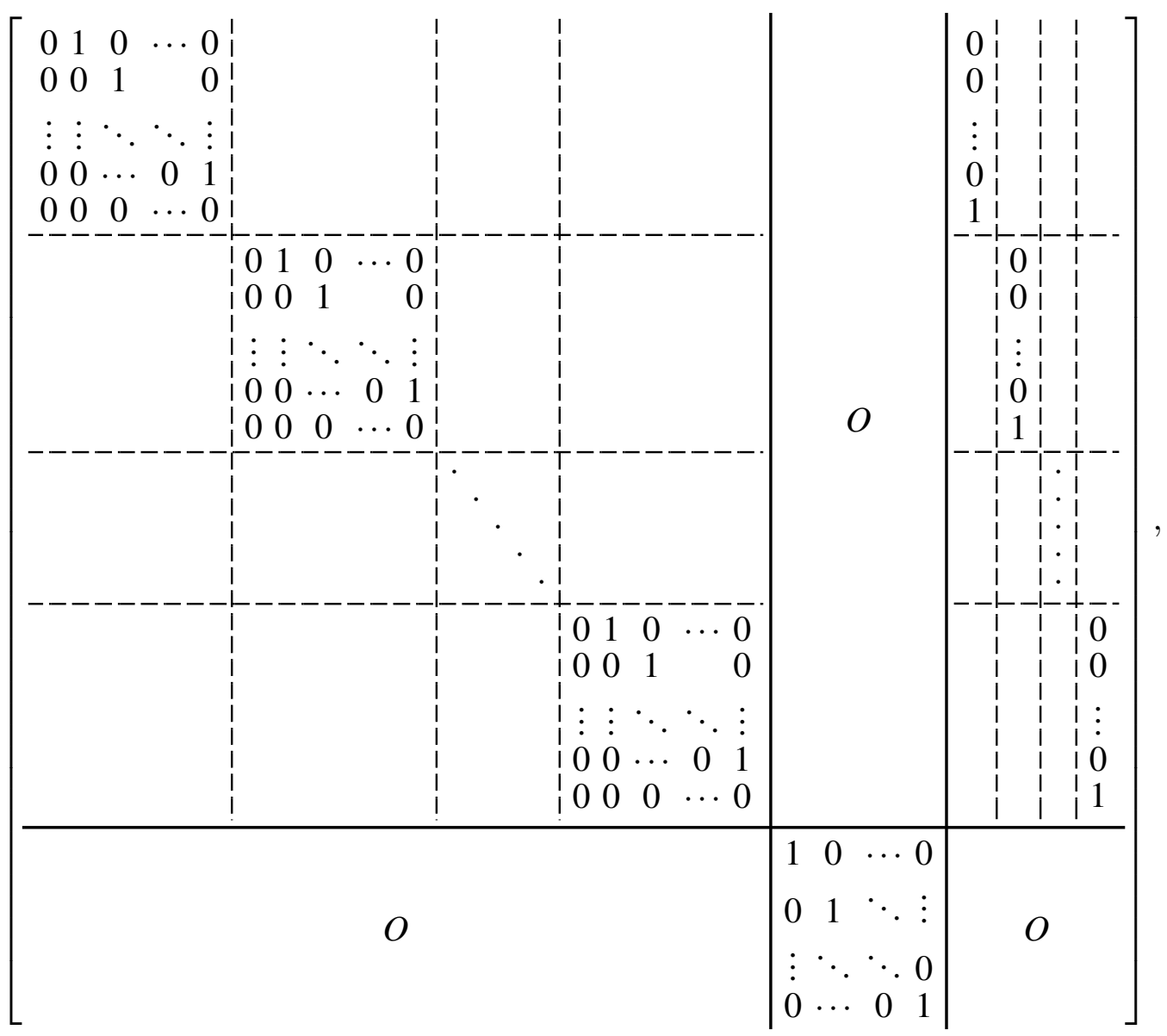

which clearly has $n^{\prime}+m$ independent rows, so the matrix $\left[A_{1}^{*}-0 \cdot I B^{*}\right]$ is full rank.

When $\lambda=1$, we have 


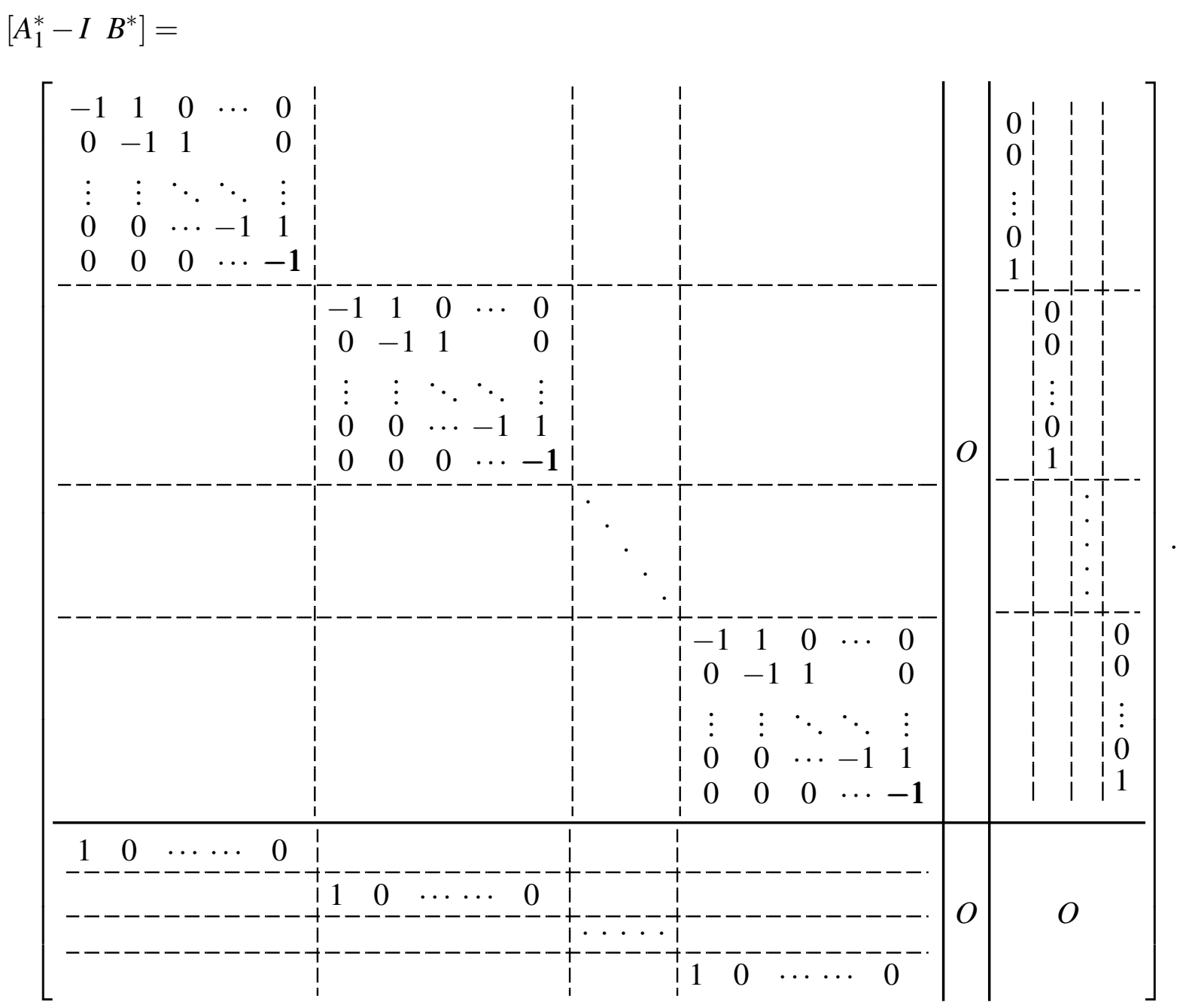

Performing elementary row/column operations, the bold -1 's can be cancelled, leaving the ma- 


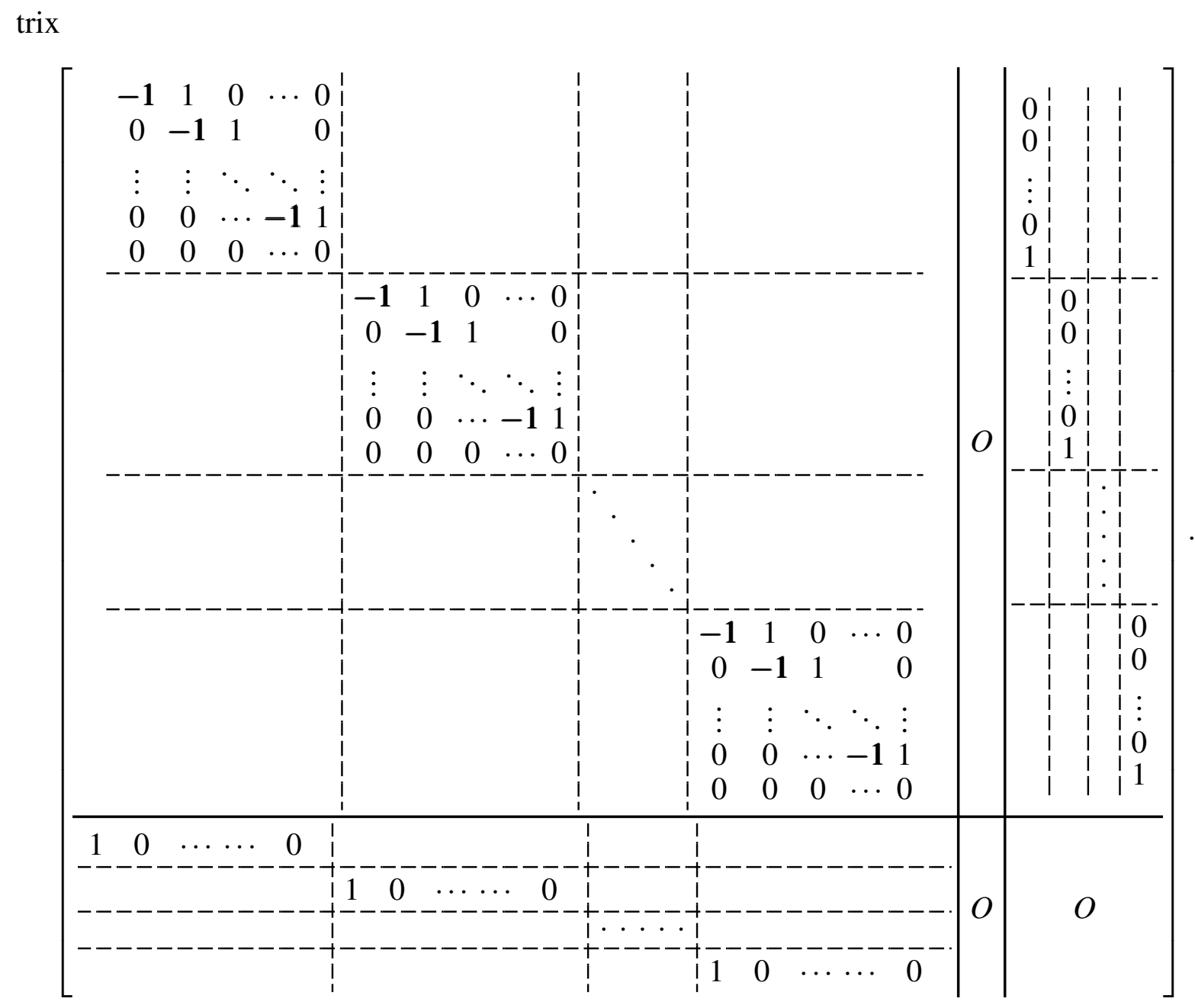


Further row/column operations yield the matrix

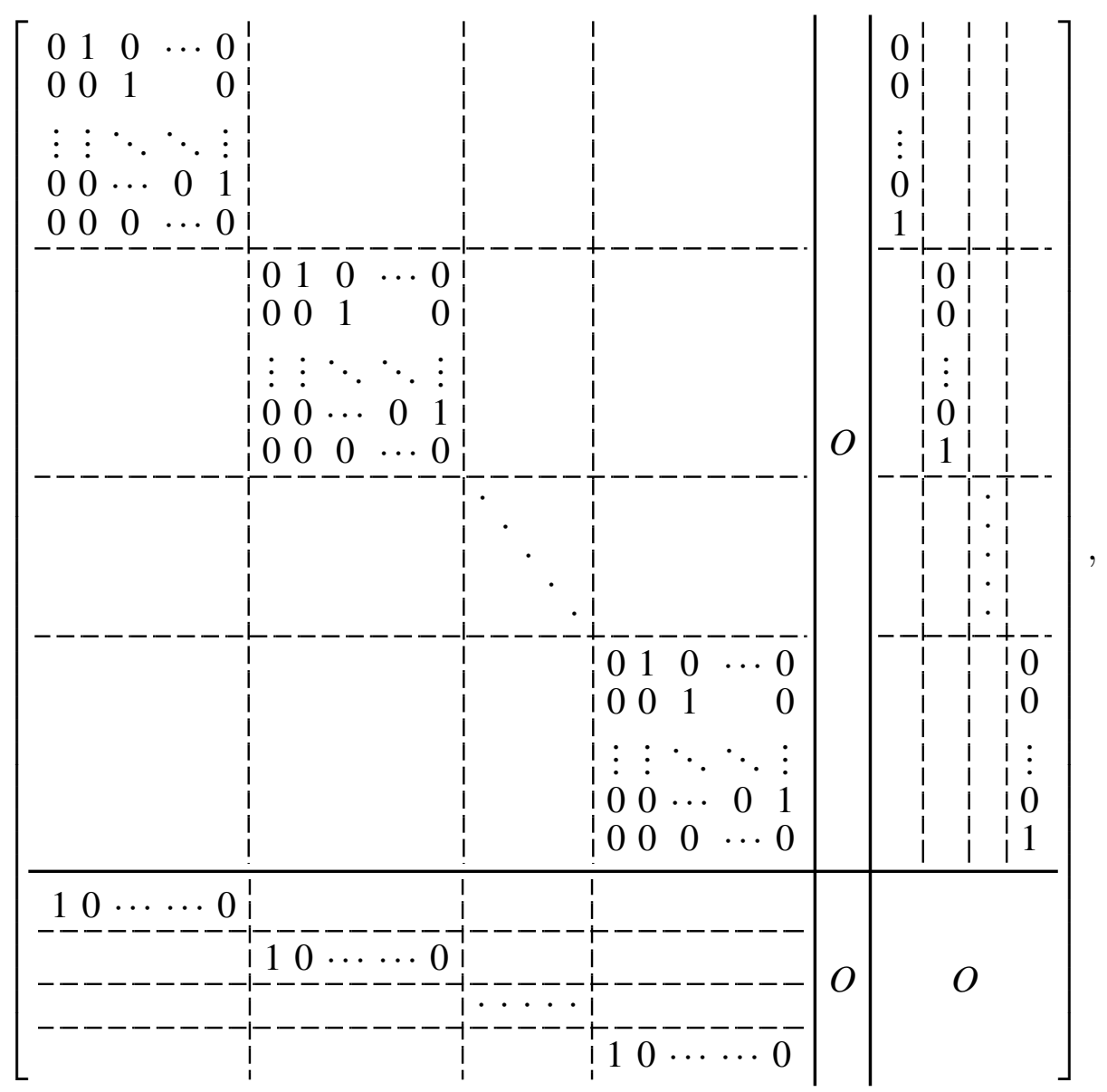

in which again there are $n^{\prime}+m$ independent rows, so the matrix $\left[A_{1}^{*}-I B^{*}\right]$ is full rank.

In conclusion, the matrix $\left[\begin{array}{lll}A_{1}^{*}-\lambda I & B^{*}\end{array}\right]$ is full rank for both $\lambda=0$ or 1 , so the augmented system is controllable. 


\section{Appendix B: Proof of Lemma 3}

Using the matrices $A_{1}^{*}$ and $B^{*}$ from Appendix A and the $K$ matrix from 5.26) -5.27 , we have $A_{1}^{*}-B^{*} K=$

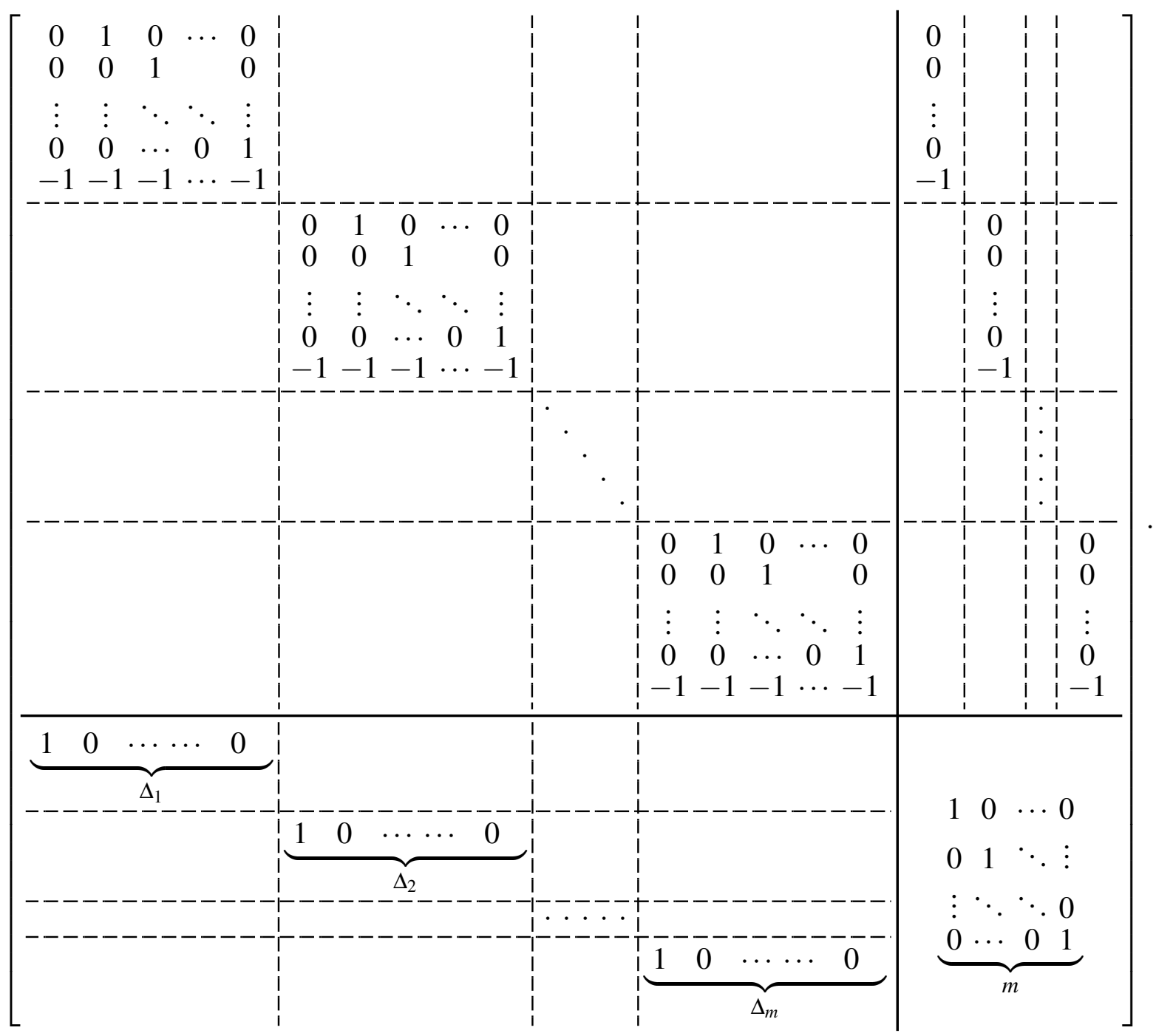


To determine the eigenvalues of matrix $A_{1}^{*}-B^{*} K$, we need to find $\lambda$ which ensures that

$$
\left|\lambda I-\left(A_{1}^{*}-B^{*} K\right)\right|=0 .
$$

The matrix $\lambda I-\left(A_{1}^{*}-B^{*} K\right)$ is

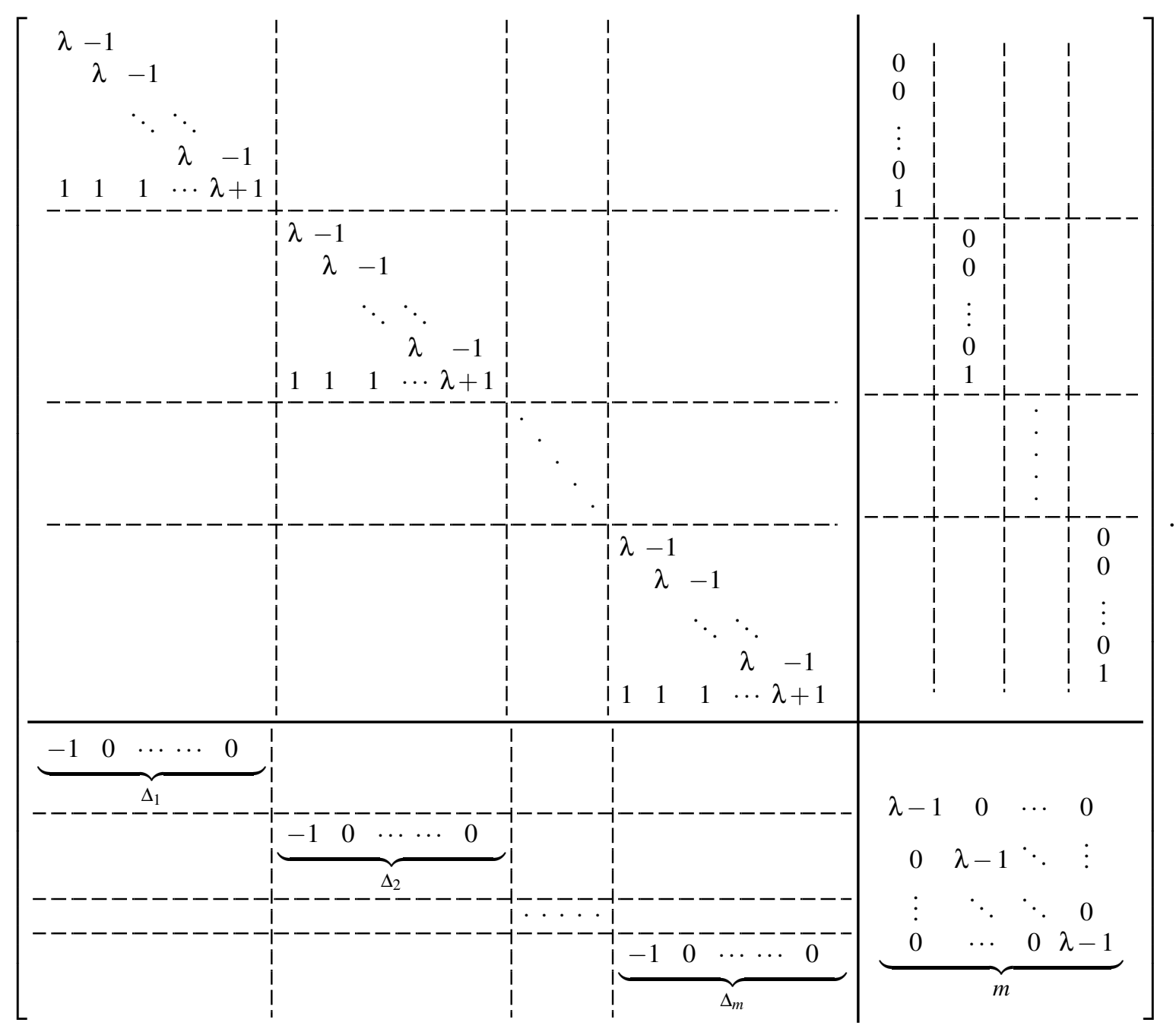


After some elementary row/column operations, the matrix becomes

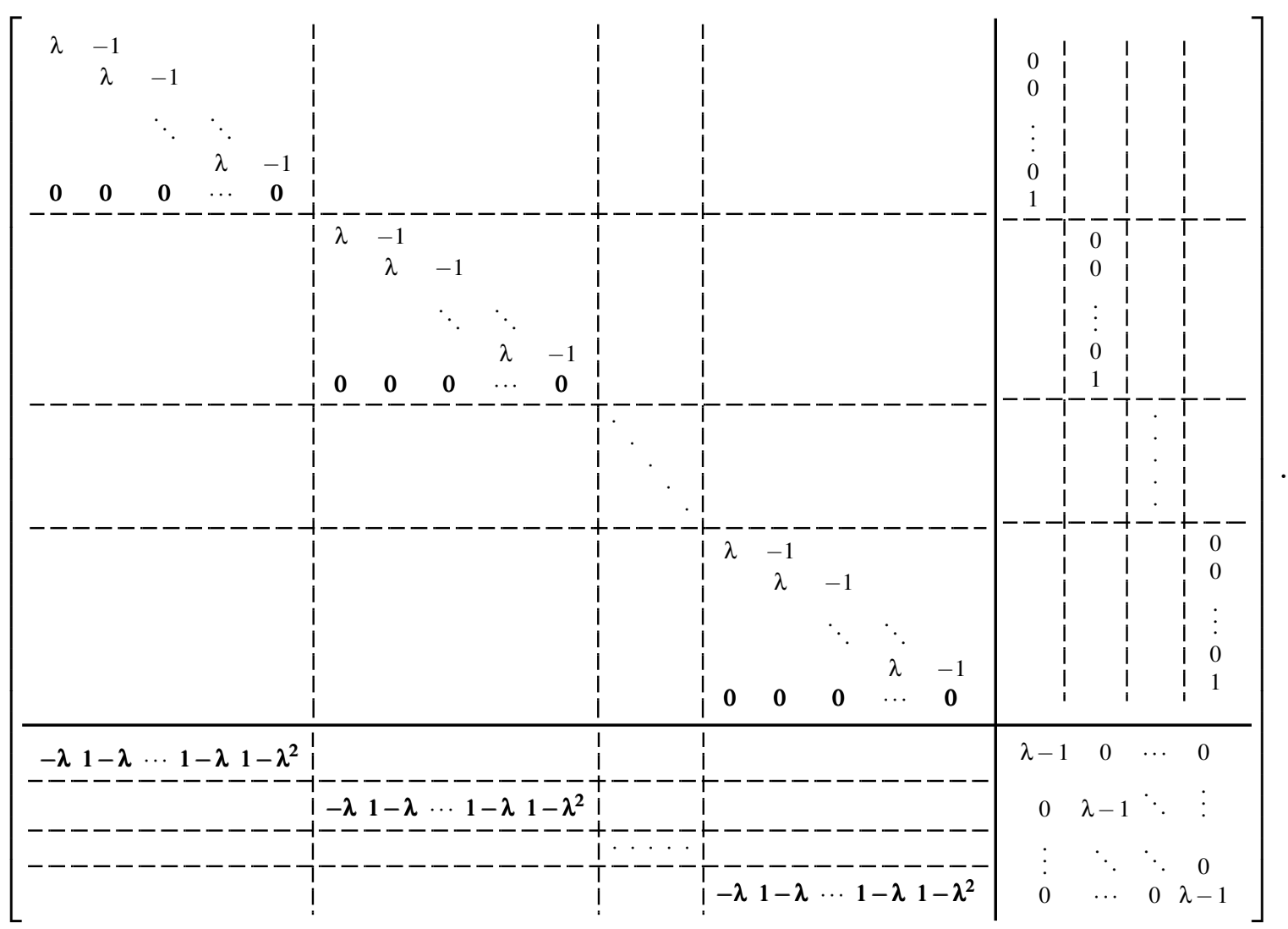

After some additional column operations for the two blocks on the left side, the matrix then 


\section{becomes}

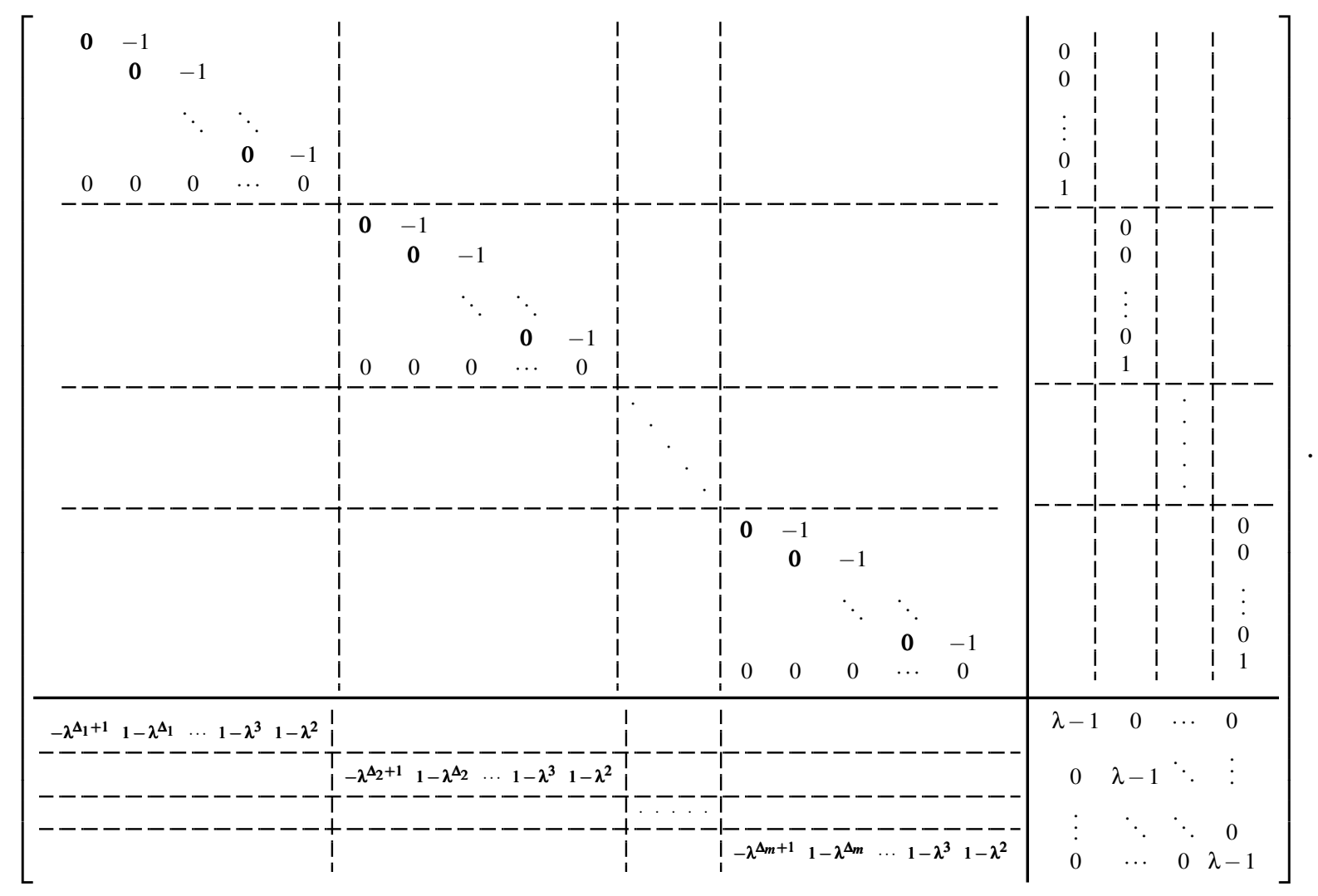


Finally, after some elementary row operations, we have

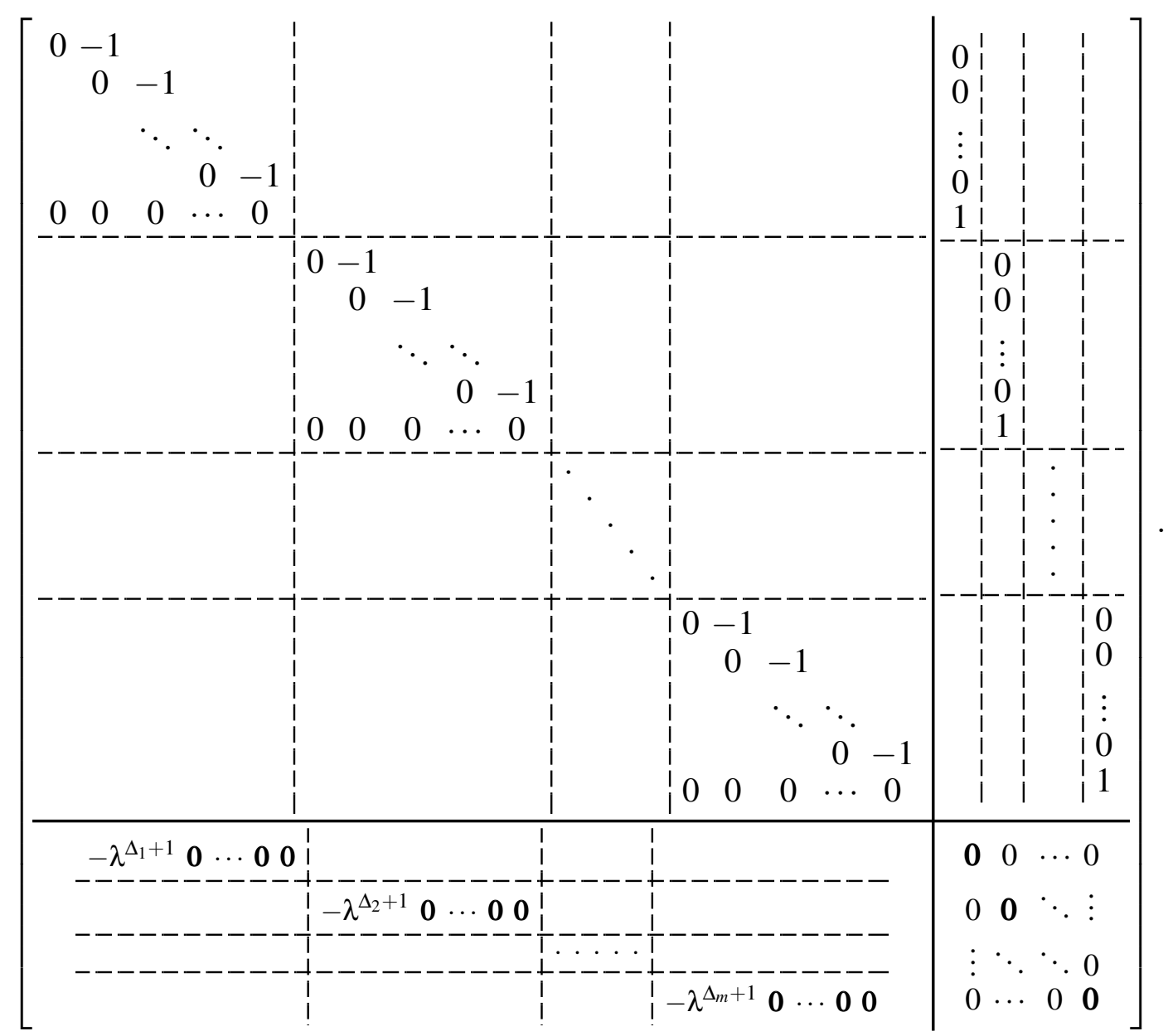

It is obvious that the matrix is full rank except when $\lambda=0$. This implies that all eigenvalues of matrix $A_{1}^{*}-B^{*} K$ are zero. Hence, the choice of $K$ in (5.26)-(5.27) ensures that the closed-loop eigenvalues of the augmented system $5.23-5.24$ are all at the origin. 


\section{Appendix C: Matlab Code for the System in Figure 5.4}

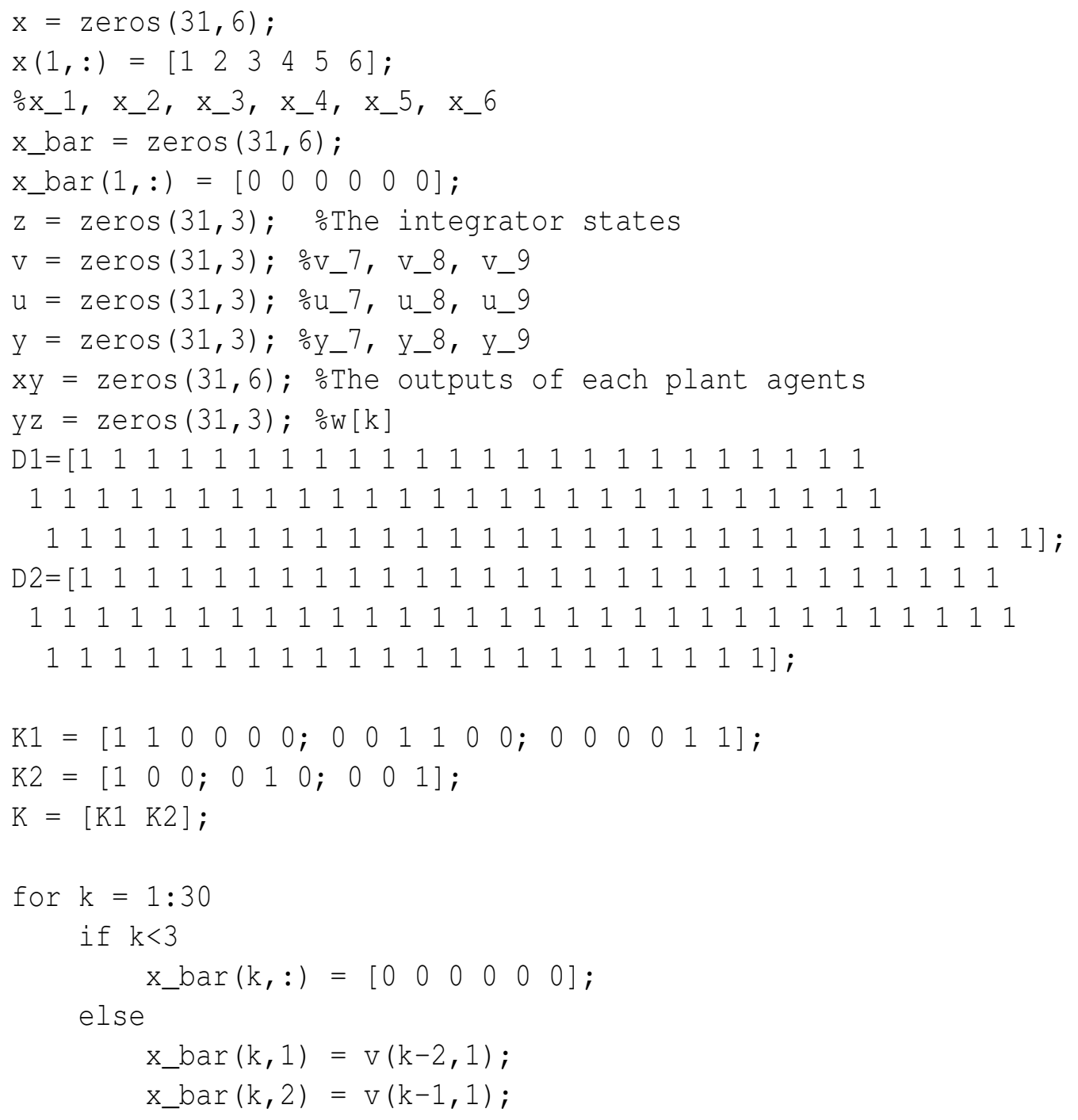




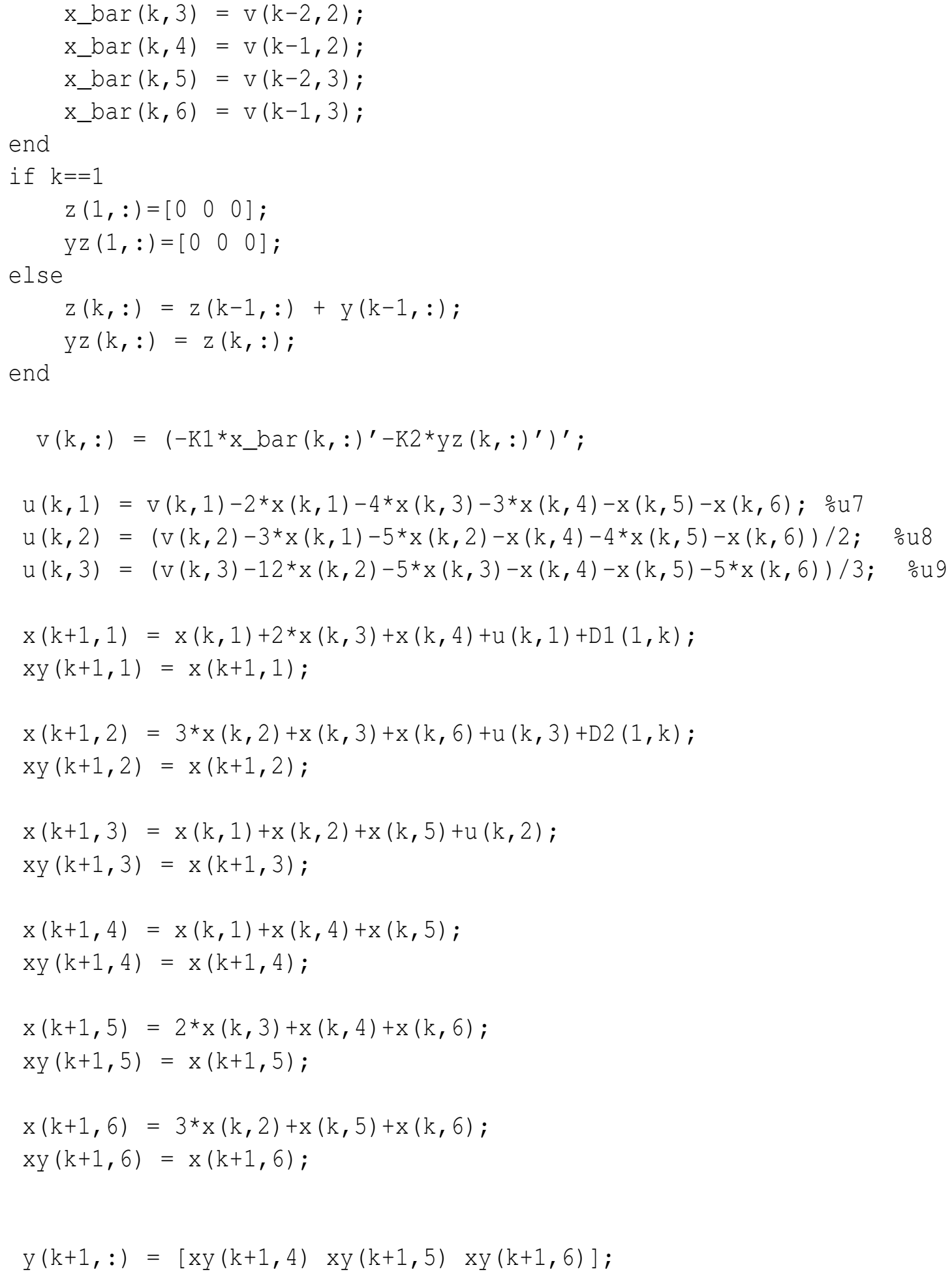




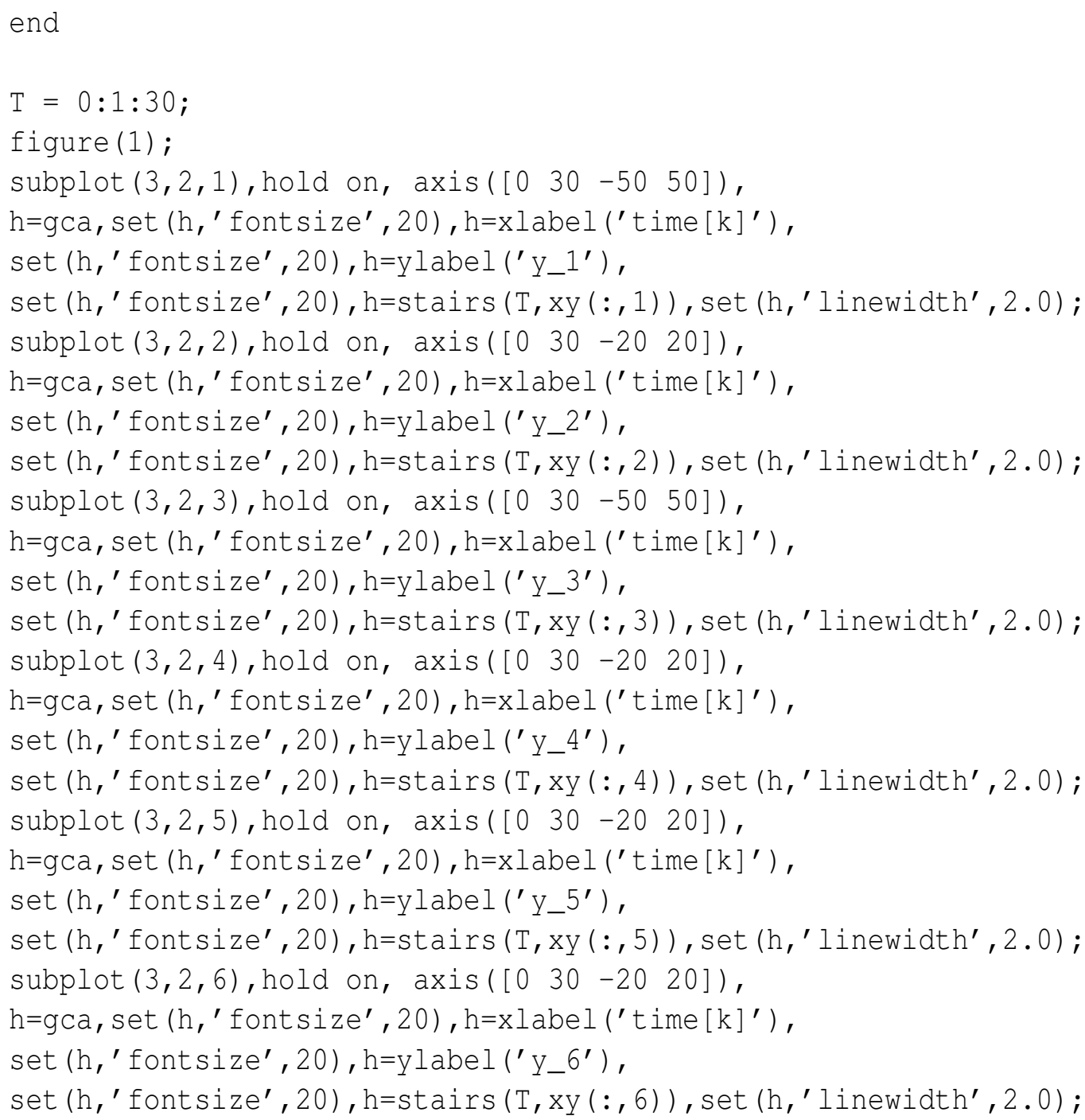




\section{Appendix D: Matlab Code for the System in Figure 5.10}

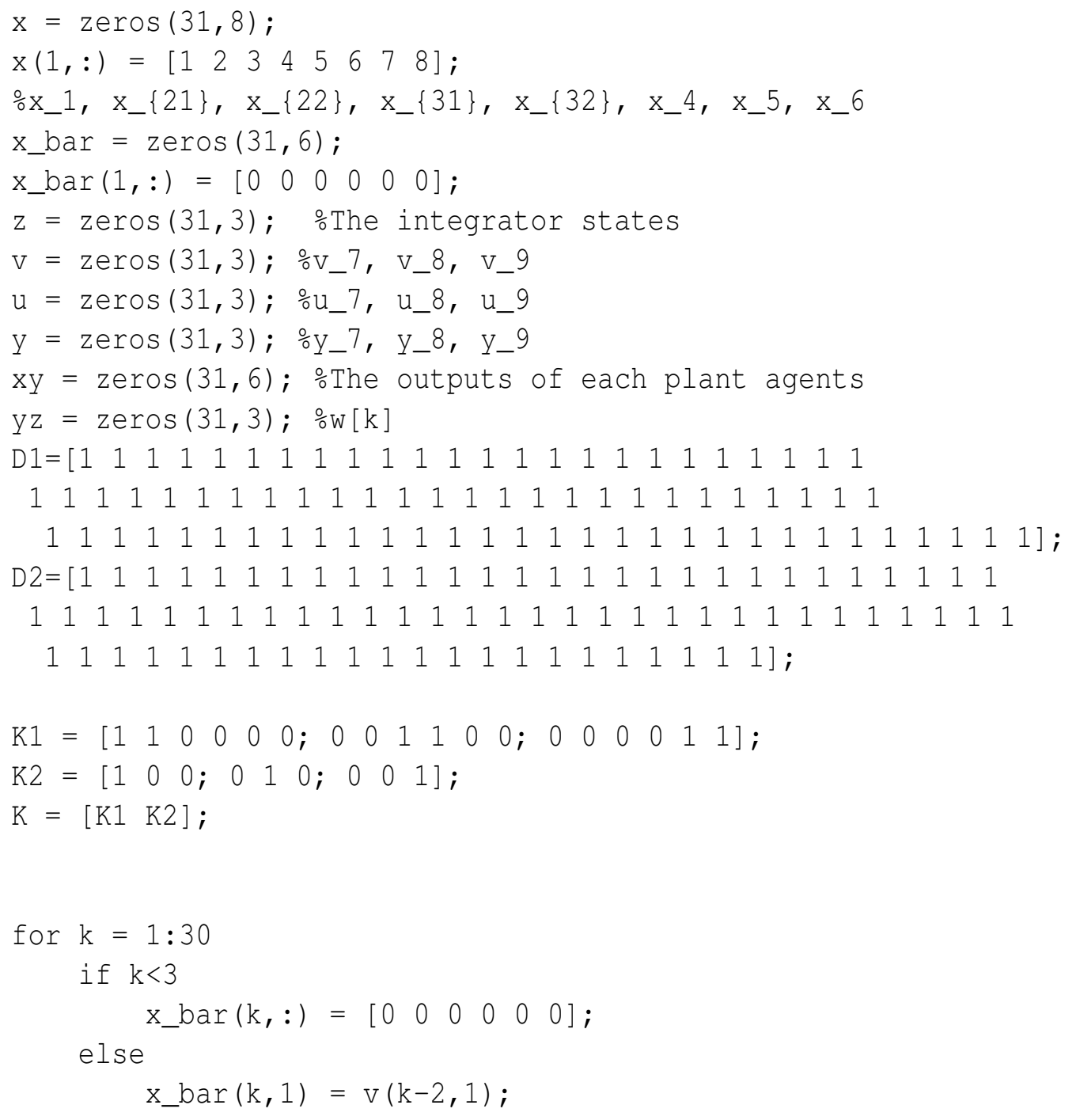




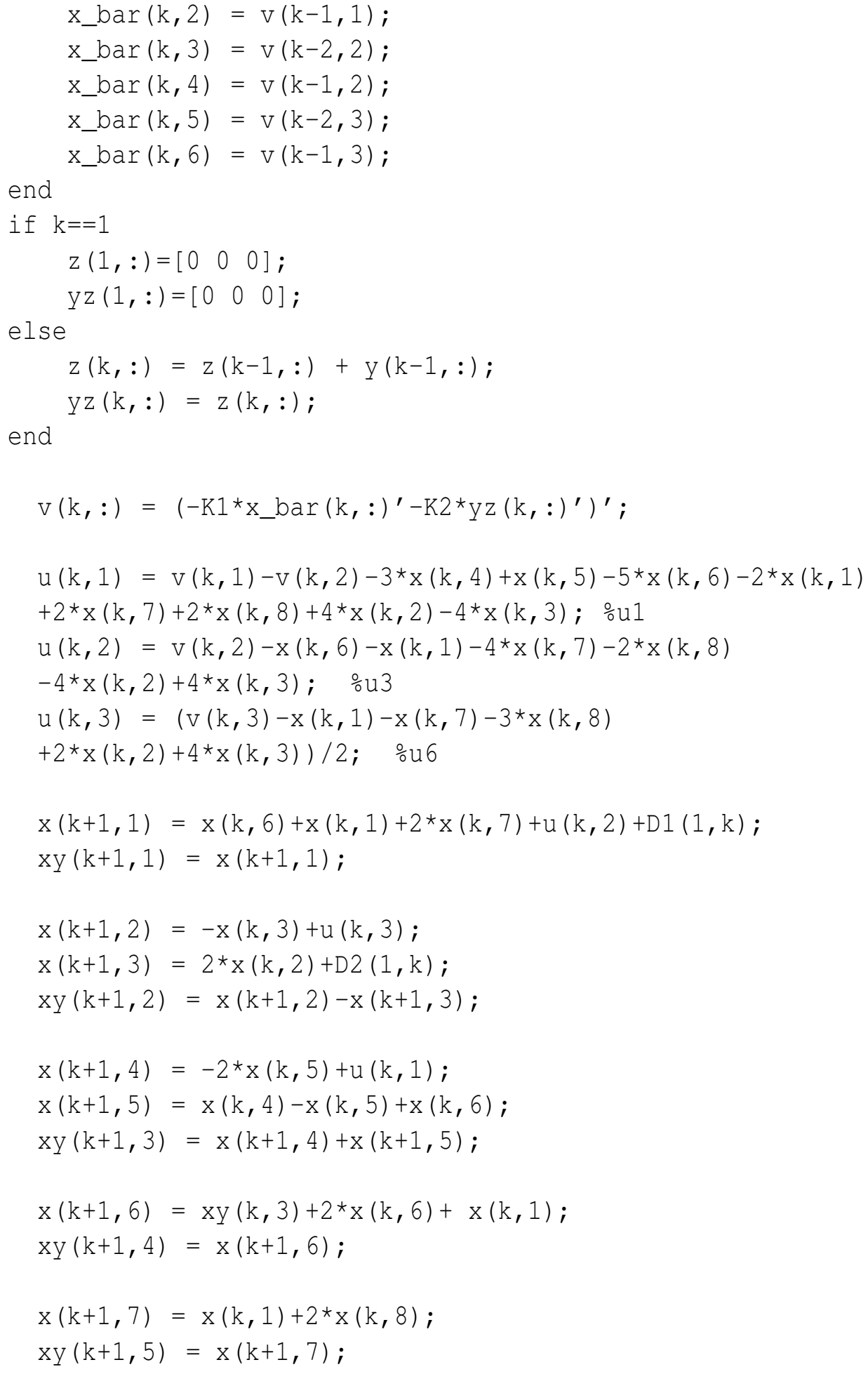




$$
\begin{aligned}
& x(k+1,8)=x(k, 7)+x(k, 8)+2 * x y(k, 2) ; \\
& x y(k+1,6)=x(k+1,8) ; \\
& y(k+1,:)=[x y(k+1,4) x y(k+1,5) x y(k+1,6)] ;
\end{aligned}
$$

end

$\mathrm{T}=0: 1: 30$;

figure (1);

subplot $(3,2,1)$, hold on, axis ([0 $30-50$ 50]),

h=gca, set (h,' fontsize', 20), h=xlabel ('time [k]'),

set (h,' fontsize', 20), h=ylabel ('y_1'),

set (h,' fontsize', 20), h=stairs $(T, x y(:, 1))$, set (h,' linewidth', 2.0);

subplot $(3,2,2)$, hold on, axis([0 $30-20$ 20]),

h=gca, set (h,' fontsize', 20), h=xlabel ('time [k]'),

set ( $h$,' fontsize', 20), h=ylabel ('y_2'),

set $\left(h,{ }^{\prime}\right.$ fontsize', 20), h=stairs $(\mathrm{T}, \mathrm{xy}(:, 2))$, set $\left(\mathrm{h},{ }^{\prime}\right.$ linewidth', 2.0) ;

subplot $(3,2,3)$, hold on, axis $\left(\left[\begin{array}{llll}0 & 30 & -50 & 50\end{array}\right]\right)$,

$h=g c a$, set $(h, '$ fontsize' 20$), h=x l a b e l('$ time $[k] ')$,

set $(h, '$ fontsize', 20), h=ylabel ('y_3'),

set $\left(h, '\right.$ fontsize', 20), h=stairs $(\mathrm{T}, \mathrm{xy}(:, 3))$, set $\left(\mathrm{h},{ }^{\prime}\right.$ linewidth', 2.0) ;

subplot $(3,2,4)$, hold on, axis ([0 $30-20$ 20]),

h=gca, set (h,' fontsize', 20), h=xlabel ('time [k]'),

set ( $h, '$ fontsize', 20), h=ylabel ('y_4'),

set $\left(\mathrm{h},{ }^{\prime}\right.$ fontsize', 20), h=stairs $(\mathrm{T}, \mathrm{xy}(:, 4))$, set $\left(\mathrm{h},{ }^{\prime}\right.$ linewidth', 2.0$)$;

subplot $(3,2,5)$, hold on, axis ([0 $30-20$ 20]),

h=gca, set (h,' fontsize', 20), h=xlabel ('time [k]'),

set (h,' fontsize', 20), h=ylabel ('y_5'),

set $(h, '$ fontsize', 20), h=stairs $(T, x y(:, 5))$, set (h,' linewidth', 2.0);

subplot $(3,2,6)$, hold on, axis([0 $30-20$ 20]),

$h=g c a$, set $(h, '$ fontsize', 20$), h=x l a b e l('$ time $[k] ')$,

set ( $h$,' fontsize', 20), h=ylabel ('y_6'),

$\operatorname{set}\left(\mathrm{h},{ }^{\prime}\right.$ fontsize', 20), h=stairs $(\mathrm{T}, \mathrm{xy}(:, 6)), \operatorname{set}\left(\mathrm{h},{ }^{\prime}\right.$ linewidth', 2.0) ; 


\section{Bibliography}

[1] M. Aoki. On feedback stabilizability of decentralized dynamic systems. Automatica, 1972. 2

[2] K. Bergey, Kevin Spieser, and D. E. Davison. The psychological dynamics of students in a classroom: Modeling and control strategies based on suggestibility theory. In Conference on Control Applications, Singapore, October 2007. IEEE.

[3] Chi-Tsong Chen. Linear System Theory and Design. Oxford University Press, 2009. 89

[4] J. Corfmat and A. Morse. Decentralized control of linear multivariable systems. Automatica, 12, 1976. 2

[5] J. Shamma (editor). Cooperative control of distributed multi-agent systems. 2007. 2

[6] J. Feddema, C. Lewis, and D. Schoenwald. Decentralized control of cooperative robotic vehicles: Theory and application. IEEE Transactions on Robotics and Automation, 18(5), 2002. 2

[7] Z. Gao. Active disturbance rejection control: a paradigm shift in feedback control system design. American Control Conference, 2006. 2

[8] T. Gustavi, D. Dimarogonas, M. Egerstedt, and X. Hu. Sufficient conditions for connectivity maintenance and rendezvous in leader-follower networks. Automatica, 46(1), 2010. 2

[9] J.B. Hoagg. Deadbeat internal model control for command following and disturbance rejection in discrete-time systems. American Control Conference, 2006. 2

[10] W. S. Levine. The Control Handbook. CRC Press, 1996.

[11] R. Olfati-Saber, J. Fax, and R. Murray. Consensus and cooperation in networked multiagent systems. Proceedings of the IEEE, 95(1), 2007. 2

[12] Richard M. Phelan. Automatic Control Systems. Cornell University Press, 1977. 
[13] Z. Qu. Cooperative Control of Dynamical Systems: Applications to Autonomous Vehicles. Springer, 2009. 2

[14] W. Ren and R. Beard. Distributed consensus in multi-vehicle cooperative control: Theory and applications. 2008. 2

[15] Nasim A. Shams. Decentralized regulation of nonlinear discrete-time multi-agent systems. MASc thesis, University of Waterloo, 2011. 3, 11, 12, 13, 15, 17

[16] Nasim A. Shams and Daniel E. Davison. A graph-theoretic condition necessary for the stabilization of crowds using cooperating control agents. In IEEE Canadian Conference on Electrical and Computer Engineering, Calgary, Canada, May 2010. 1

[17] Nasim A. Shams and Daniel E. Davison. A co-operative control approach to the regulation of nonlinear discrete-time structured systems. In IEEE Canadian Conference on Electrical and Computer Engineering, Niagara Falls, Canada, May 2011. vii, 1, 6, 7, 8, 11, 14, 15, 16, 17, 21, 22

[18] D.D. Siljak. Decentralized Control of Complex Systems. Academic Press, 1991. 2

[19] K. Spieser and D. E. Davison. Multi-agent stabilization of the psychological dynamics of one-dimensional crowds. Automatica, 45, 2009. 1

[20] Kevin Spieser. Stabilizing the psychological dynamics of people in a crowd. MASc thesis, University of Waterloo, 2008. 1

[21] Kevin Spieser and D. E. Davison. Stabilizing the psychological dynamics of people in a queue. In Proceedings of the American Control Conference, Seattle, WA, June 2008. AACC.

[22] S. Sundaram and C. N. Hadjicostis. Distributed function calculation via linear iterative strategies in the presence of malicious agents. IEEE Transactions on Automatic Control, 56(7), 2011. 2

[23] S. Wang and E.J. Davison. On the stabilization of decentralized control systems. IEEE Transactions on Automatic Control, AC-18, 1973. 2

[24] Xiaoli Wang. A distributed control approach to a robust output regulation problem for multi-agent linear systems. IEEE Transactions on Automatic Control, 55(12), 2010. 2

[25] D. Yua, J. Lam, and Z. Wang. Persistent disturbance rejection via state feedback for networked control systems. Chaos, Solitons And Fractals, 40(1), 2009. 2 